\title{
The ROSAT all-sky survey catalogue of optically bright main-sequence stars and subgiant stars $^{\star}$
}

\author{
M. Hünsch ${ }^{1,3}$, J.H.M.M. Schmitt ${ }^{2,3}$, and W. Voges ${ }^{3}$ \\ 1 Institut für Theoretische Physik und Astrophysik, Universität Kiel, Olshausenstrasse 40, 24118 Kiel, Germany \\ 2 Hamburger Sternwarte, Universität Hamburg, Gojenbergsweg 112, 21029 Hamburg, Germany \\ 3 Max-Planck-Institut für Extraterrestrische Physik, Giessenbachstrasse, 85740 Garching, Germany
}

Received February 18; accepted April 14, 1998

\begin{abstract}
We present X-ray data for all main-sequence and subgiant stars of spectral types A, F, G, and $\mathrm{K}$ and luminosity classes IV and V listed in the Bright Star Catalogue that have been detected as X-ray sources in the ROSAT all-sky survey; several stars without luminosity class are also included. The catalogue contains 980 entries yielding an average detection rate of 32 percent. In addition to count rates, source detection parameters, hardness ratios, and X-ray fluxes we also list X-ray luminosities derived from Hipparcos parallaxes.
\end{abstract}

Key words: stars: activity — stars: coronae — stars: late-type - X-rays: stars - catalogs

\section{Introduction}

$\mathrm{X}$-ray emission from late-type stars is generally attributed to magnetically heated stellar coronae. As suggested by the example of the Sun's corona, the emitting hot $\left(>10^{6} \mathrm{~K}\right)$ plasma is believed to be confined by coronal magnetic fields, which ultimately originate from the interaction between rotation and outer convection zones. The solar X-ray luminosity varies approximately between $310^{25}$ and $110^{27} \mathrm{erg} \mathrm{s}^{-1}$ (extrapolated to a $1-300 \AA$ "bolometric X-ray band"; cf. Haisch \& Schmitt 1996; Acton 1996) during the solar cycle and is known to be strongly correlated with other magnetic activiy indicators (e.g., sunspot numbers, flare frequency, chromospheric CaII emission). Hence, it can be regarded as a good activity indicator also for other stars.

Send offprint requests to: M. Hünsch, e-mail: huensch@astrophysik.uni-kiel.de

* The catalogue is also available in electronic form via anonymous ftp to cdsarc.u-strasbg.fr (130.79.128.5) or via http://cdsweb.u-strasbg.fr/Abstract.html
In principle, any late-type star should be able to sustain a corona, and henceforth, be an X-ray source. Indeed, already the Einstein Observatory detected X-ray emission from a large number of late-type stars (cf. Vaiana et al. 1981; Maggio et al. 1987). Detailed studies of the immediate solar environment (Schmitt et al. 1995; Schmitt 1997) revealed that virtually every late-type dwarf star with spectral type later than A7 can be detected as an X-ray source given data of sufficient sensitivity; Schmitt (1997) argues that late-type stars emit X-rays at least at a level such that the apparent X-ray surface flux $F_{\mathrm{x}}$ exceeds $\approx 10^{4} \mathrm{erg} \mathrm{cm}^{-2} \mathrm{~s}^{-1}$. On the other hand, stellar X-ray luminosities appear to be bounded above by the so-called saturation limit $L_{\mathrm{x}} / L_{\mathrm{bol}} \sim 10^{-3}$, which can be observed both for field stars and stars in open clusters, where one observes that the spectral type above which stars appear to be saturated moves toward later types with increasing age. This is probably related to the angular momentum evolution of young late-type main-sequence stars, which are spun down by magnetic breaking during their first $\sim 10^{8}$ yrs on the main-sequence. Enhanced activity can, however, last on much longer timescales, as indicated by example of the Hyades cluster.

Until now, the ROSAT observatory has undertaken the only sensitive X-ray survey (RASS) of the whole sky. These data provide a flux-limited but otherwise unbiased sample of X-ray sources (Voges et al. 1996a). Of the $\approx 1.510^{5}$ detected sources, about one third are considered to be coronal. Many of these coronal X-ray sources have optically faint counterparts which require optical followup observations. A smaller number of X-ray sources have bright optical counterparts, and we have systematically searched for X-ray emission from these optically bright stars. The results of our survey are presented as catalogs of X-ray data, which have already been published for OB stars (Berghöfer et al. 1996) and late-type giants and supergiants (Hünsch et al. 1998; hereafter HSV98). 


\section{RASS data and detection of late-type stars}

\subsection{The ROSAT all-sky survey (RASS)}

During it's first half year of operations, the ROSAT observatory carried out the first all-sky survey with an imaging X-ray telescope between July 1990 and January 1991. Further survey observations were carried out in February 1991 (2 days) and August 1991 (10 days). The whole sky was scanned along great circles perpendicular to the direction to the Sun. Because of the Earth's motion around the Sun, the plane of these circles slowly rotated around an axis through the ecliptic poles, thus covering the whole sphere within 6 months. Each point of the sky was observed several times as the scan paths of 2 degrees width (i.e., the field of view of the PSPC detector) progressed along the ecliptic. Therefore, the data of any particular source consist of a number of "snapshots" of up to $30 \mathrm{~s}$ duration, separated by the orbital period of the satellite $(\approx 90 \mathrm{~min})$ and distributed over an interval of at least 2 days. Towards the ecliptic poles, the cumulative exposure time increases due to the larger number of scans covering a particular position. Depending on the ecliptic latitude (and down-time due to radiation belts of the Earth), the effective exposure time varies between $\sim 100 \mathrm{~s}$ and $\sim 40000 \mathrm{~s}$ (at the poles), with typical values of $\sim 400 \mathrm{~s}$ on the ecliptic. Given a typical energy-conversion factor for soft sources of $61^{-12} \mathrm{erg} \mathrm{cts}^{-1} \mathrm{~cm}^{-2}$ (cf. Sect. 2.4) the typical detection limit of RASS observations (i.e., $\approx 0.015 \mathrm{cts} \mathrm{s}^{-1}$ ) amounts to $f_{\mathrm{x}} \approx 10^{-13} \mathrm{erg} \mathrm{cm}^{-2} \mathrm{~s}^{-1}$. For a more detailed description of the RASS we refer to Voges (1992) and Belloni et al. (1994). Details of the ROSAT observatory in general can be found in Trümper (1983) and Trümper et al. (1991), the PSPC detector used during the RASS is described by Pfeffermann et al. (1986).

In February 1997 the remaining gaps left in the all sky survey were filled with a sequence of pointed, partially overlapping PSPC observations so that with the exception of a small region around the strong X-ray source Sco X-1 the whole sky has been imaged with the ROSAT PSPC. In the catalog presented in this paper we include sources detected in this "survey repair" pointed observations; they are marked with an asterisk.

The source detection was performed by means of a maximum likelihood algorithm (Cruddace et al. 1988) in the course of the standard analysis software system (SASS; Voges et al. 1992). The significance of an X-ray source is expressed by the likelihood $\mathrm{Li}=-\ln (1-P)$, where $P$ is the probability of existence; e.g., a likelihood of $\mathrm{Li}=7$ corresponds to a source existence probability of $99.9 \%$. The result of the SASS is a comprehensive list of several $10^{4}$ sources, each source described by the sky position in right ascension and declination, its source detection likelihood, count rate, hardness ratio, extent, and corresponding errors. The data for the brighter X-ray sources have recently been released as the ROSAT All-sky Survey Bright Source
Catalogue (Voges et al. 1996b), which contains sources with Likelihood $\geq 15$, count rate larger than $0.05 \mathrm{~s}^{-1}$, and with at least 15 detected photons.

\subsection{Selection of stars}

We used the Bright Star Catalogue (BSC; Hoffleit \& Warren 1991) as input sample for our search of X-ray bright late-type stars. In particular, we extracted all stars of spectral types A, F, G, and K and luminosity classes IV and V (including subtypes like IV-V, IVa, but not III-IV). Note that there are no M-type stars in this sample except for the MV star HR 1703, which is obviously a misclassified giant, according to its Hipparcos parallax. We also included those stars lacking an MK classification but with a suffix "d" or "sg", and the composite-spectrum stars that do not have one or both companions classified as a giant (these are treated in HSV98). Finally, we included stars of the above mentioned spectral types but without any indication of luminosity (no MK type, no suffix). This also holds for the many Ap-, Am-, Fp- and Fm-stars. Thus in total, our input sample consists of 3054 stars.

One has to keep in mind that because of the large spread in absolute magnitude of main-sequence stars, very different space volumes are covered by our magnitudelimited sample. While A-type stars are almost completely covered by the BSC up to a distance of $\approx 50 \mathrm{pc}, \mathrm{K}$-types stars are only listed in the BSC if they are within a few pc from the Sun. This selection effect obviously introduces a strong bias to any derived X-ray luminosity function.

About 1 percent of the sky was not included in the original RASS, and we list in Table 1 those stars with less than $50 \mathrm{~s}$ exposure time. Note that the stars HR 813 ( $\mu$ Cet), HR 997, HR 5234, HR 5325, and HR 5568 were detected in the pointed survey repair observations and are included in Table 2.

\subsection{Matches between input stars and RASS-sources}

The procedure whereby the positions of RASS sources were matched with the stars of our input sample has been extensively described in HSV98. Here, we only report that we have accepted sources with a likelihood greater than 7 within 90 arcsec distance from the input stars. The choice of this cut-off radius is justified by means of a Monte Carlo simulation of 10000 random positions, i.e., approximately the same number as our BSC input catalog; see HSV98 for details. That means, at 90 arcseconds offset between optical and X-ray position the probability that the $\mathrm{X}$-ray source can be attributed to the star (and not to a background object) is $50 \%$. This differential probability increases very rapidly for smaller values of positional offset (see Fig. 2 in HSV98).

For about $400 \mathrm{X}$-ray sources extracted in this way and not included in the Bright Source Catalogue (Voges et al. 
Table 1. Stars of the input sample with less than $50 \mathrm{~s}$ exposure time or which are located in the region of the X-ray bright Vela supernova remnant. Stars marked with ${ }^{*}$ in the last column were detected in the survey repair

\begin{tabular}{|c|c|c|c|c|}
\hline HR & Name & Sp. type & $V$ & \\
\hline 479 & & F5IV-V & 6.84 & \\
\hline 486 & & K0V & 5.86 & \\
\hline 487 & & $\mathrm{~K} 5 \mathrm{~V}$ & 5.82 & \\
\hline 506 & & $\mathrm{~F} 8 \mathrm{~V}$ & 5.52 & \\
\hline 563 & $\iota$ Ari & $\mathrm{K} 1 \mathrm{Vp}$ & 5.10 & \\
\hline 729 & 26 Ari & A9V & 6.15 & \\
\hline 797 & & $\mathrm{~A} 2 \mathrm{~V}$ & 6.30 & \\
\hline 813 & $\mu$ Cet & FOIV & 4.27 & $*$ \\
\hline 892 & & A2IV & 5.23 & \\
\hline 925 & $\rho^{3}$ Eri & A $8 \mathrm{~V}$ & 5.26 & \\
\hline 997 & & FOIV & 5.71 & $*$ \\
\hline 1053 & & A $3-5 V+G 0-5$ & 6.15 & \\
\hline 1064 & & F3-5IV & 5.96 & \\
\hline 1082 & & A3V & 6.38 & \\
\hline 1114 & $\tau$ For & AOV & 6.01 & \\
\hline 2820 & $1 \mathrm{CMi}$ & A5IV & 5.30 & \\
\hline 2836 & & A2IV & 6.37 & \\
\hline 2893 & & A1V & 6.28 & \\
\hline 2952 & & A0 & 6.59 & \\
\hline 2953 & & K0 & 6.24 & \\
\hline 2987 & & K0 & 6.43 & \\
\hline 3008 & $11 \mathrm{CMi}$ & A1Vnn & 5.30 & \\
\hline 3030 & & K0 & 6.04 & \\
\hline 3064 & 9 Pup & G0V: & 5.17 & \\
\hline 3104 & & Ko & 5.99 & \\
\hline 3394 & & FOIV & 6.32 & \\
\hline 3510 & $54 \mathrm{Cnc}$ & G1V & 6.38 & \\
\hline 3530 & & $\mathrm{~A} 1 \mathrm{Vn}$ & 6.55 & \\
\hline 3551 & & A9IV-V & 5.33 & \\
\hline 3588 & & $\mathrm{Am}$ & 5.18 & \\
\hline 4028 & & A9IV & 6.55 & \\
\hline 4043 & & A $5 \mathrm{~V}$ & 6.22 & \\
\hline 4054 & 40 Leo & F6IV & 4.79 & \\
\hline 4070 & 42 Leo & A1V & 6.12 & \\
\hline 4148 & 49 Leo & $\mathrm{A} 2 \mathrm{~V}$ & 5.67 & \\
\hline 4579 & & A0-1V & 6.43 & \\
\hline 5234 & & $\mathrm{~A} 1 \mathrm{~V}$ & 6.14 & * \\
\hline 5325 & & F9V & 6.31 & * \\
\hline 5474 & & $\mathrm{ApSi}$ & 6.37 & \\
\hline 5568 & & $\mathrm{~K} 4 \mathrm{~V}$ & 5.74 & * \\
\hline 5639 & & dG6 & 6.10 & \\
\hline 7067 & & K5 & 6.17 & \\
\hline 7086 & & $\mathrm{~A} 1 \mathrm{~V}$ & 5.88 & \\
\hline 7205 & & F5IV-V & 6.37 & \\
\hline 7393 & $\mu \mathrm{Tel}$ & F5V & 6.30 & \\
\hline 7546 & $\zeta$ Sge & A3V & 5.00 & \\
\hline 7579 & & $\mathrm{Am}$ & 5.75 & \\
\hline 7833 & & $\mathrm{~A} 3 \mathrm{~m}$ & 6.55 & \\
\hline 7839 & & $\mathrm{~A} 1 \mathrm{~m}$ & 6.18 & \\
\hline 7981 & & A1Vs & 6.52 & \\
\hline 8372 & & $\mathrm{~K} 5 \mathrm{~V}$ & 6.38 & \\
\hline 8586 & $39 \mathrm{Peg}$ & F1V & 6.42 & \\
\hline 8624 & $41 \mathrm{Peg}$ & $\mathrm{A} 2 \mathrm{~V}$ & 6.21 & \\
\hline 8756 & & A5V & 6.28 & \\
\hline 8959 & & $\mathrm{~A} 2 \mathrm{~V}$ & 4.74 & \\
\hline 8960 & $74 \mathrm{Peg}$ & $\mathrm{A} 1 \mathrm{~V}$ & 6.26 & \\
\hline 8963 & $75 \mathrm{Peg}$ & $\mathrm{A} 1 \mathrm{Vn}$ & 5.40 & \\
\hline
\end{tabular}

1996b), we checked the X-ray images by eye for reality. Specifically, we rejected photon distributions that are significantly contaminated by nearby strong sources or that are obviously extended. In questionable cases, we ran the standard source detection algorithm of EXSAS on the source images in different passbands and decided on the basis of the results which sources to retain in our final catalog.
Confining now attention to the 3054 BSC positions identified with late-type main-sequence stars and subgiant stars, we detected X-ray emission from 980 stars, i.e., the average detection rate is $32 \%$. Since the total search area around these 3054 stars is $3054 \cdot \pi \cdot\left(1.5^{\prime}\right)^{2}=6.00 \square^{\circ}=$ $0.0145 \%$ of the sphere, and the total number of RASS sources amounts to $\sim 150000$, we would expect 21.8 chance coincidences of late-type main-sequence stars or subgiants with background (or foreground) X-ray sources (i.e., $2.2 \%$ of our detected sources).

\subsection{Determination of X-ray fluxes and luminosities}

The procedure of determining X-ray fluxes has also been described in HSV98. In this paper, we followed the same procedure, except using a slightly different formula for the calculation of individual energy-conversion factors

$\mathrm{ECF}=(5.30 \cdot h r+8.31) \quad 10^{-12} \mathrm{erg} \mathrm{cm}^{-2} \mathrm{cts}^{-1}$

which was derived by Schmitt et al. (1995) from an X-ray study of a complete sample of the nearby main-sequence stars; here $h r$ denotes the hardness ratio defined through $h r=\frac{\mathrm{H}-\mathrm{S}}{\mathrm{H}+\mathrm{S}}$,

where $\mathrm{H}$ and $\mathrm{S}$ denote the source counts in the hard $(0.5-$ $2.0 \mathrm{keV})$ and soft $(0.1-0.4 \mathrm{keV})$ passbands of ROSAT. The hardness ratio is an "X-ray colour" that is influenced by both the plasma temperature and the hydrogen column density.

Since the SASS source detection was separately performed in both passbands, and since most of our X-ray sources were detected in both bands, the hardness ratios can be estimated for many stars, although in some cases with quite substantial errors. In a few cases, when the sources were not detected in either the soft or the hard passband, we set $h r=+1.0$ or -1.0 by definition, respectively. We refrain from estimating individual errors for $f_{\mathrm{x}}$ since the error in ECF is very difficult to quantify. In general, we estimate this error to be within a factor of two for the weaker sources and less for the brighter sources. The $\mathrm{X}$-ray luminosities are calculated using the recently available Hipparcos parallaxes. We only accepted those parallaxes which exceed their corresponding error by at least a factor of 3. Fortunately, this is the case for almost all of the input stars. Where this criterion is not fulfilled, the distance and X-ray luminosity column entries in Table 2 are left open. The X-ray luminosities are then calculated by the relation

$L_{\mathrm{x}}=4 \pi d^{2} \times f_{\mathrm{x}}$,

where $d$ is the distance to the star.

\section{The catalogue}

Table 2 contains optical and X-ray data of all 980 detected late-type main-sequence stars and subgiant stars as contained in the BSC and specified in Sect. 2.2. 
The columns of the table contain the following information:

Col. 1: HR number (BSC). An asterisk indicates objects detected in the survey repair observations (see Sect. 2.2). Col. 2: HD number.

Col. 3: the star's name (Bayer or Flamsteed designation). Col. 4: $V$ magnitude (from BSC).

Col. 5: $B-V$ colour index (from BSC).

Col. 6: MK spectral classification (from BSC).

Col. 7: Distance to the star as given by the Hipparcos parallaxes.

Col: 8: binary flag; S: single star, VB: visual binary (if a companion within 90 arcseconds distance is known), SB: spectroscopic binary (as given in the BSC), B: other binary (composite spectrum).

Col. 9: effective exposure time in seconds.

Col. 10: mean PSPC count rate in counts per second.

Col. 11: error of PSPC count rate.

Col. 12: likelihood of existence (cf. Sect. 2.1).

Col. 13: offset in arcseconds between optical and X-ray position.

Col. 14: hardness ratio $h r=(\mathrm{H}-\mathrm{S}) /(\mathrm{H}+\mathrm{S})$ (cf. Sect. 2.4).

Col. 15: error of hardness ratio.

Col. 16: apparent X-ray flux $(0.1-2.4 \mathrm{keV})$ in $10^{-14} \mathrm{erg} \mathrm{cm}^{-2} \mathrm{~s}^{-1}$ (see Sect. 2.4).

Col. 17: X-ray luminosity derived from the distance as given in Col. 7. The values are given in units of $10^{27} \mathrm{erg} \mathrm{s}^{-1}$.

Acknowledgements. The ROSAT project is supported by the Bundesministerium für Bildung, Forschung und Technologie (BMBF/DARA) and the Max-Planck-Gesellschaft (MPG). We would like to thank our colleagues from the MPE ROSAT group, especially T. Berghöfer, for their support.

\section{References}

Acton L.W., 1996, in: Pallavicini R., Dupree A.K. (eds.) Cool Stars, Stellar Systems, and the Sun, Proc. 9th Cambridge Workshop, ASP Conf. Ser. 109, 45

Belloni T., Hasinger G., Izzo, C., 1994, A\&A 283, 1037

Berghöfer T.W., Schmitt J.H.M.M., Cassinelli J.P., 1996, A\&AS 118, 481

Cruddace R.G., Hasinger G.R., Schmitt J.H.M.M., 1988, The application of a maximum likelihood analysis to detection of sources in the ROSAT data, in: Murtagh F., Heck A. (eds.) Astronomy from Large Databases, ESO Conf. and Workshop Proc. 28, p. 177

Haisch B.M., Schmitt J.H.M.M., 1996, PASP 108, 113

Hoffleit D,E., Warren W.H.jr., 1991, The Bright Star Catalogue, 5th Rev. (ed.), Yale Univ. Obs., New Haven

Hünsch M., Schmitt J.H.M.M., Voges W., 1998, A\&AS 127, 251 (HSV98)

Maggio A., Sciortino S., Vaiana G.S., et al., 1987, ApJ 315, 687

Pfeffermann E., Briel U.G., Hippmann U., et al., 1986, Proc. SPIE 733, 519

Schmitt J.H.M.M., 1997, A\&A 318, 215

Schmitt J.H.M.M., Fleming T.A., Giampapa M.S., 1995, ApJ 450,392

Trümper J., 1983, Adv. Space Res. 2, No. 4, 241

Trümper J., et al., 1991, Nat 349, 579

Vaiana G.S., Cassinelli J.P., Fabbiano G., et al., 1981, ApJ 244,163

Voges W., 1992, in: Proc. of the ISY Conf. "Space Science", ESA ISY-3, ESA Publ., p. 9

Voges W., et al., 1992, in: Proc. of the ISY Conf. "Space Science", ESA ISY-3, ESA Publ., p. 223

Voges W., et al., 1996a, MPE Report 263, 637

Voges W., et al., 1996b, IAUC 6420 
Table 2. (See Sect. 3)

\begin{tabular}{|c|c|c|c|c|c|c|c|c|c|c|c|c|c|c|c|c|}
\hline HR & HD & name & $V$ & $B-V$ & MK type & dist & binary & Exp. & $\mathrm{CR}$ & $\pm \mathrm{CR}$ & $\mathrm{Li}$ & $\Delta$ & $h r$ & $\Delta h r$ & $f_{\mathrm{x} 14}$ & $L_{\mathrm{x} 27}$ \\
\hline 5 & 123 & & 5.96 & 0.67 & G5V & 20.3 & VB & 508 & 0.323 & 0.026 & 409 & 4 & -0.33 & 0.08 & 212.0 & 104.4 \\
\hline 8 & 166 & & 6.13 & 0.75 & $\mathrm{KOV}$ & 13.7 & $\mathrm{~S}$ & 327 & 0.687 & 0.048 & 624 & 8 & -0.21 & 0.06 & 494.3 & 111.0 \\
\hline 9 & 203 & & 6.18 & 0.38 & A7V & 39.1 & $\mathrm{~S}$ & 313 & 0.044 & 0.015 & 13 & 24 & 0.32 & 0.31 & 44.1 & 80.6 \\
\hline 32 & 661 & & 6.64 & 0.37 & $\mathrm{~F} 2 \mathrm{~V}+\mathrm{F} 6 \mathrm{~V}$ & 66.4 & VB & 568 & 0.023 & 0.009 & 9 & 38 & -0.42 & 0.30 & 14.1 & 74.1 \\
\hline 41 & 905 & 23 And & 5.72 & 0.31 & F0IV & 35.0 & $\mathrm{~S}$ & 367 & 0.042 & 0.012 & 26 & 13 & 0.11 & 0.28 & 37.4 & 54.7 \\
\hline 50 & 1061 & $35 \mathrm{Psc}$ & 5.79 & 0.31 & F0IV & 80.2 & SB & 634 & 0.039 & 0.009 & 36 & 9 & -0.22 & 0.21 & 28.1 & 216.0 \\
\hline 56 & 1185 & & 6.15 & 0.05 & $\mathrm{~A} 2 \mathrm{VpSi}$ & 93.0 & VB & 422 & 0.015 & 0.007 & 8 & 20 & -1.00 & 0.87 & 20.3 & 210.0 \\
\hline 66 & 1343 & & 6.45 & 0.37 & F4IV-V & 48.9 & $\mathrm{~S}$ & 326 & 0.019 & 0.009 & 9 & 44 & -1.00 & 0.43 & 26.1 & 74.6 \\
\hline 88 & 1835 & 9 Cet & 6.39 & 0.66 & G2V & 20.4 & $\mathrm{~S}$ & 321 & 0.289 & 0.033 & 209 & 12 & -0.29 & 0.10 & 195.7 & 97.3 \\
\hline 98 & 2151 & $\beta$ Hyi & 2.80 & 0.62 & G2IV & 7.5 & $\mathrm{~S}$ & 195 & 0.070 & 0.023 & 15 & 19 & -1.00 & 0.25 & 95.1 & 6.4 \\
\hline 104 & 2421 & & 5.17 & 0.03 & $\mathrm{~A} 2 \mathrm{~V} \mathrm{~s}$ & 81.2 & SB & 421 & 0.032 & 0.011 & 14 & 34 & 0.52 & 0.30 & 35.7 & 281.7 \\
\hline 108 & 2475 & & 6.43 & 0.59 & G0V & 32.2 & VB & 341 & 0.176 & 0.025 & 98 & 3 & -0.39 & 0.13 & 110.0 & 136.9 \\
\hline 120 & 2726 & & 5.69 & 0.35 & $\mathrm{~F} 2 \mathrm{~V}$ & 45.1 & $\mathrm{~S}$ & 193 & 2.241 & 0.110 & 999 & 8 & 0.13 & 0.04 & 2016.7 & 4900.2 \\
\hline 140 & 3158 & & 5.57 & 0.47 & F3IV-V & 25.6 & $\mathrm{~S}$ & 216 & 0.037 & 0.015 & 12 & 38 & -1.00 & 0.31 & 50.4 & 39.6 \\
\hline 142 & 3196 & $13 \mathrm{Cet}$ & 5.20 & 0.56 & F8V & 21.0 & $\mathrm{SB}, \mathrm{VB}$ & 421 & 1.116 & 0.076 & 588 & 3 & -0.20 & 0.06 & 809.1 & 428.9 \\
\hline 143 & 3229 & $14 \mathrm{Cet}$ & 5.93 & 0.44 & F5IV & 55.6 & $\mathrm{~S}$ & 553 & 0.119 & 0.017 & 115 & 7 & -0.13 & 0.13 & 90.8 & 336.6 \\
\hline 147 & 3302 & $\lambda^{2} \mathrm{Phe}$ & 5.51 & 0.44 & F6V & 36.2 & $\mathrm{~S}$ & 184 & 0.224 & 0.039 & 61 & 17 & -0.22 & 0.16 & 160.2 & 251.5 \\
\hline 172 & 3794 & & 6.49 & 0.92 & G5 & 154.3 & $\mathrm{~S}$ & 223 & 0.199 & 0.033 & 81 & 12 & 0.29 & 0.15 & 196.4 & 5597.4 \\
\hline 187 & 4089 & $\rho$ Tuc & 5.39 & 0.50 & F6V & 40.7 & SB & 106 & 0.118 & 0.038 & 27 & 4 & 0.14 & 0.35 & 106.9 & 211.5 \\
\hline 191 & 4150 & $\eta$ Phe & 4.36 & 0.00 & A0IV & 73.7 & VB & 189 & 0.060 & 0.022 & 12 & 23 & -0.27 & 0.32 & 41.5 & 270.0 \\
\hline 196 & 4222 & & 5.42 & 0.04 & $\mathrm{~A} 2 \mathrm{~V} \mathrm{~s}$ & 107.8 & $\mathrm{~S}$ & 469 & 0.027 & 0.010 & 10 & 37 & -0.06 & 0.34 & 21.8 & 303.1 \\
\hline 200 & 4295 & & 6.33 & 0.00 & F3V & 40.6 & $\mathrm{~S}$ & 595 & 0.024 & 0.008 & 15 & 22 & -0.17 & 0.29 & 17.8 & 35.0 \\
\hline 209 & 4391 & & 5.80 & 0.64 & G1V & 14.9 & VB & 215 & 0.335 & 0.044 & 130 & 7 & -0.39 & 0.11 & 208.9 & 55.8 \\
\hline 219 & 4614 & $\eta$ Cas & 3.44 & 0.57 & $\mathrm{~F} 9 \mathrm{~V}+\mathrm{dM} 0$ & 6.0 & $\mathrm{SB}, \mathrm{VB}$ & 381 & 0.138 & 0.022 & 71 & 17 & -0.72 & 0.11 & 62.0 & 2.6 \\
\hline 222 & 4628 & & 5.75 & 0.88 & $\mathrm{~K} 2 \mathrm{~V}$ & 7.5 & $\mathrm{~S}$ & 353 & 0.043 & 0.013 & 17 & 30 & -1.00 & 0.16 & 58.7 & 3.9 \\
\hline 225 & 4676 & $64 \mathrm{Psc}$ & 5.07 & 0.51 & F8V & 23.9 & SB & 418 & 0.024 & 0.009 & 11 & 9 & -0.72 & 0.28 & 10.7 & 7.4 \\
\hline 235 & 4813 & $\varphi^{2}$ Cet & 5.19 & 0.50 & F7IV-V & 15.5 & $\mathrm{~S}$ & 565 & 0.073 & 0.013 & 54 & 8 & -0.71 & 0.11 & 33.1 & 9.5 \\
\hline 244 & 5015 & & 4.82 & 0.53 & F8V & 18.6 & SB & 326 & 0.044 & 0.014 & 12 & 28 & -0.71 & 0.24 & 19.9 & 8.2 \\
\hline 251 & 5156 & & 6.46 & 0.44 & F6IV-V & 74.5 & VB & 328 & 0.038 & 0.013 & 12 & 38 & 0.17 & 0.31 & 35.1 & 232.8 \\
\hline 252 & 5190 & $\lambda^{1}$ Tuc & 6.22 & 0.56 & F7IV-V & 61.2 & $\mathrm{VB}$ & 288 & 0.020 & 0.011 & 8 & 35 & -1.00 & 0.75 & 27.9 & 125.2 \\
\hline 297 & 6210 & & 5.84 & 0.54 & F6V & 80.2 & $\mathrm{~S}$ & 428 & 0.052 & 0.013 & 29 & 15 & 0.36 & 0.24 & 53.0 & 408.0 \\
\hline 301 & 6288 & 26 Cet & 6.04 & 0.27 & F1V & 56.6 & VB & 418 & 0.034 & 0.012 & 13 & 6 & 0.17 & 0.31 & 31.4 & 120.5 \\
\hline 303 & 6301 & & 6.19 & 0.43 & F7IV-V & 42.0 & $\mathrm{~S}$ & 447 & 0.073 & 0.014 & 50 & 7 & 0.12 & 0.19 & 65.4 & 137.9 \\
\hline 305 & 6314 & & 6.72 & 0.31 & FoVn & 84.4 & $\mathrm{~S}$ & 452 & 0.049 & 0.012 & 29 & 7 & -0.19 & 0.23 & 36.1 & 307.4 \\
\hline 313 & 6479 & 77 Psc & 6.35 & 0.38 & F3V & 45.7 & VB & 325 & 0.055 & 0.016 & 21 & 17 & -0.58 & 0.21 & 29.0 & 72.6 \\
\hline 314 & 6480 & $77 \mathrm{Psc}$ & 7.25 & 0.49 & F6V & 44.1 & VB & 325 & 0.055 & 0.016 & 21 & 24 & -0.58 & 0.21 & 29.0 & 67.4 \\
\hline 325 & 6668 & & 6.37 & 0.23 & A7V & 71.6 & $\mathrm{~S}$ & 335 & 0.038 & 0.013 & 15 & 26 & -0.39 & 0.28 & 24.0 & 147.2 \\
\hline 327 & 6680 & $78 \mathrm{Psc}$ & 6.25 & 0.40 & F5IV & 41.9 & $\mathrm{~S}$ & 453 & 0.080 & 0.015 & 48 & 5 & -0.21 & 0.19 & 57.4 & 120.6 \\
\hline 328 & 6695 & $\psi^{2} \mathrm{Psc}$ & 5.55 & 0.13 & A3V & 49.4 & $\mathrm{~S}$ & 241 & 0.069 & 0.019 & 26 & 5 & -0.22 & 0.27 & 49.2 & 143.9 \\
\hline 329 & 6706 & 30 Cet & 5.82 & 0.43 & F7IV & 47.7 & $\mathrm{~S}$ & 247 & 0.047 & 0.016 & 16 & 20 & 0.22 & 0.31 & 44.5 & 121.2 \\
\hline 340 & 6920 & 44 And & 5.65 & 0.60 & F8V & 52.7 & $\mathrm{~S}$ & 384 & 0.107 & 0.018 & 50 & 26 & 0.04 & 0.18 & 91.0 & 302.3 \\
\hline 357 & 7238 & & 6.26 & 0.42 & F5V s & 72.3 & $\mathrm{~S}$ & 734 & 0.094 & 0.013 & 93 & 15 & 0.06 & 0.13 & 81.2 & 507.1 \\
\hline 358 & 7259 & & 6.52 & 0.48 & F7IV & 71.2 & $\mathrm{~S}$ & 331 & 0.048 & 0.014 & 25 & 19 & 0.01 & 0.29 & 39.9 & 242.1 \\
\hline 361 & 7344 & $\zeta$ Psc & 5.24 & 0.32 & A7IV & 45.3 & $\mathrm{SB}, \mathrm{VB}$ & 421 & 0.093 & 0.016 & 73 & 32 & -0.26 & 0.15 & 64.5 & 158.2 \\
\hline 362 & 7345 & $\zeta \mathrm{Psc}$ & 6.30 & 0.49 & F7V & 45.3 & $\mathrm{SB}, \mathrm{VB}$ & 421 & 0.093 & 0.016 & 73 & 10 & -0.26 & 0.15 & 64.5 & 158.2 \\
\hline 366 & 7439 & $37 \mathrm{Cet}$ & 5.13 & 0.46 & F5V & 24.4 & VB & 335 & 0.126 & 0.021 & 84 & 13 & -0.01 & 0.16 & 104.0 & 74.0 \\
\hline 370 & 7570 & $\nu \mathrm{Phe}$ & 4.96 & 0.58 & F8V & 15.1 & $\mathrm{~S}$ & 324 & 0.039 & 0.013 & 16 & 10 & -1.00 & 0.47 & 52.7 & 14.3 \\
\hline 377 & 7788 & $\kappa$ Tuc & 4.86 & 0.47 & F6IV & 20.4 & VB & 110 & 0.522 & 0.072 & 116 & 14 & -0.40 & 0.12 & 322.9 & 161.3 \\
\hline 378 & 7804 & $89 \mathrm{Psc}$ & 5.16 & 0.07 & A3V & 67.4 & SB & 421 & 0.017 & 0.008 & 7 & 61 & 0.02 & 0.45 & 14.4 & 78.3 \\
\hline 391 & 8272 & & 6.45 & 0.00 & F4V & 57.7 & VB & 379 & 0.076 & 0.017 & 42 & 3 & -0.24 & 0.20 & 53.8 & 214.2 \\
\hline 396 & 8375 & & 6.29 & 0.83 & G8IV & 58.9 & $\mathrm{~S}$ & 354 & 0.065 & 0.018 & 22 & 48 & 0.36 & 0.25 & 66.7 & 276.9 \\
\hline 398 & 8424 & & 6.49 & 0.00 & A0Vnn & 157.7 & $\mathrm{~S}$ & 695 & 0.012 & 0.005 & 9 & 26 & -0.05 & 0.44 & 9.8 & 292.1 \\
\hline 404 & 8556 & & 5.91 & 0.41 & F3V & 44.8 & $\mathrm{SB}, \mathrm{VB}$ & 445 & 0.041 & 0.012 & 16 & 13 & -0.63 & 0.23 & 20.3 & 48.8 \\
\hline 405 & 8589 & & 6.14 & 0.91 & G5 & 102.5 & $\mathrm{~S}$ & 350 & 0.062 & 0.016 & 22 & 87 & -0.52 & 0.21 & 34.7 & 435.3 \\
\hline 410 & 8673 & & 6.31 & 0.47 & F7V & 38.3 & $\mathrm{~S}$ & 359 & 0.062 & 0.016 & 24 & 13 & -0.60 & 0.20 & 32.0 & 56.1 \\
\hline 413 & 8723 & $\rho \mathrm{Psc}$ & 5.38 & 0.39 & F2V: & 26.1 & $\mathrm{~S}$ & 380 & 0.195 & 0.025 & 130 & 10 & -0.17 & 0.12 & 144.5 & 117.6 \\
\hline 415 & 8774 & & 6.27 & 0.46 & F7IVb vs & 42.6 & $\mathrm{~S}$ & 369 & 0.078 & 0.017 & 44 & 8 & -0.42 & 0.18 & 47.4 & 103.1 \\
\hline 417 & 8799 & $\omega$ And & 4.83 & 0.42 & F5IV & 28.3 & $\mathrm{~S}$ & 254 & 0.254 & 0.034 & 104 & 10 & -0.30 & 0.12 & 170.6 & 163.5 \\
\hline 419 & 8803 & & 6.58 & -0.04 & $\mathrm{~B} 9 \mathrm{~V}+\mathrm{A} 8 \mathrm{~V}$ & 160.0 & VB & 426 & 0.035 & 0.010 & 18 & 18 & 0.34 & 0.27 & 35.0 & 1071.6 \\
\hline 427 & 9021 & $38 \mathrm{Cas}$ & 5.81 & 0.47 & F6V & 29.2 & $\mathrm{SB}$ & 637 & 0.077 & 0.013 & 91 & 7 & -0.22 & 0.15 & 55.3 & 56.6 \\
\hline 428 & 9030 & & 6.14 & 0.08 & $\mathrm{~A} 2 \mathrm{~V} \mathrm{~s}$ & 86.7 & SB & 570 & 0.027 & 0.008 & 19 & 14 & -0.33 & 0.27 & 17.5 & 157.4 \\
\hline 433 & 9132 & $48 \mathrm{Cet}$ & 5.12 & 0.02 & A1V & 67.9 & VB & 237 & 0.078 & 0.023 & 17 & 31 & -0.02 & 0.26 & 64.0 & 353.3 \\
\hline 447 & 9544 & & 6.28 & 0.46 & $\mathrm{~F} 4 \mathrm{~V}$ & 80.6 & $\mathrm{~S}$ & 285 & 0.067 & 0.018 & 28 & 13 & -0.23 & 0.25 & 47.5 & 369.1 \\
\hline 458 & 9826 & $v$ And & 4.09 & 0.54 & F8V & 13.5 & SB & 354 & 0.135 & 0.023 & 66 & 31 & -0.70 & 0.11 & 62.1 & 13.5 \\
\hline 462 & 9906 & $\tau \mathrm{Scl}$ & 5.69 & 0.33 & $\mathrm{~F} 2 \mathrm{~V}$ & 62.0 & VB & 512 & 0.037 & 0.011 & 21 & 6 & -0.22 & 0.27 & 26.4 & 121.2 \\
\hline 463 & 9919 & $\pi \mathrm{Psc}$ & 5.57 & 0.35 & FoV & 33.8 & $\mathrm{~S}$ & 416 & 0.042 & 0.012 & 19 & 18 & -0.56 & 0.20 & 22.7 & 31.0 \\
\hline 492 & 10453 & & 5.75 & 0.44 & $\mathrm{~F} 5 \mathrm{~V}+\mathrm{F} 7 \mathrm{~V}$ & 37.3 & VB & 425 & 0.037 & 0.011 & 21 & 18 & -0.38 & 0.25 & 23.2 & 38.5 \\
\hline 494 & 10481 & & 6.17 & 0.42 & $\mathrm{~F} 2 \mathrm{~V}$ & 41.9 & $\mathrm{~S}$ & 380 & 0.100 & 0.019 & 51 & 19 & -0.34 & 0.16 & 65.1 & 136.8 \\
\hline 499 & 10543 & & 6.21 & 0.10 & A3V & 79.2 & VB & 250 & 0.244 & 0.034 & 120 & 9 & 0.17 & 0.13 & 224.7 & 1685.7 \\
\hline 508 & 10697 & 109 Psc & 6.27 & 0.75 & G3Va & 32.6 & $\mathrm{~S}$ & 390 & 0.020 & 0.008 & 10 & 39 & -1.00 & 0.33 & 27.5 & 34.9 \\
\hline 509 & 10700 & $\tau$ Cet & 3.50 & 0.72 & G8V & 3.6 & $\mathrm{~S}$ & 445 & 0.052 & 0.013 & 24 & 11 & -1.00 & 0.08 & 70.4 & 1.1 \\
\hline 511 & 10780 & & 5.63 & 0.81 & K0V & 10.0 & $\mathrm{~S}$ & 572 & 0.360 & 0.026 & 505 & 7 & -0.60 & 0.05 & 184.8 & 22.0 \\
\hline 512 & 10800 & & 5.87 & 0.61 & G2V & 27.1 & $\mathrm{~S}$ & 291 & 0.260 & 0.032 & 156 & 7 & -0.08 & 0.12 & 204.7 & 180.3 \\
\hline 514 & 10830 & $\epsilon \mathrm{Scl}$ & 5.31 & 0.39 & F1V & 27.4 & VB & 431 & 0.260 & 0.026 & 251 & 6 & -0.32 & 0.09 & 172.2 & 155.0 \\
\hline 518 & 10874 & & 6.32 & 0.43 & F6V & 58.3 & $\mathrm{~S}$ & 305 & 0.031 & 0.013 & 7 & 17 & -0.37 & 0.40 & 19.4 & 79.0 \\
\hline 526 & 11031 & & 5.82 & 0.29 & A3V & 101.6 & $\mathrm{SB}, \mathrm{VB}$ & 326 & 0.031 & 0.012 & 9 & 30 & -0.20 & 0.37 & 22.3 & 275.9 \\
\hline 529 & 11151 & & 5.90 & 0.43 & F5IV & 44.6 & $\mathrm{~S}$ & 326 & 0.019 & 0.010 & 8 & 82 & 0.26 & 0.65 & 18.8 & 44.7 \\
\hline 534 & 11257 & & 5.94 & 0.30 & $\mathrm{~F} 2 \mathrm{~V}$ w & 42.6 & VB & 397 & 0.016 & 0.008 & 7 & 2 & -1.00 & 0.87 & 22.5 & 48.8 \\
\hline 535 & 11262 & & 6.37 & 0.52 & $\mathrm{~F} 8 \mathrm{~V}$ & 27.1 & VB & 521 & 0.025 & 0.009 & 12 & 13 & -0.29 & 0.31 & 16.9 & 14.8 \\
\hline 544 & 11443 & $\alpha$ Tri & 3.41 & 0.49 & F6IV & 19.7 & SB & 284 & 1.005 & 0.062 & 875 & 7 & -0.04 & 0.06 & 813.8 & 376.3 \\
\hline
\end{tabular}


Table 2. continued

\begin{tabular}{|c|c|c|c|c|c|c|c|c|c|c|c|c|c|c|c|c|}
\hline $\mathrm{HR}$ & HD & name & V & $B-V$ & MK type & dist & binary & Exp. & $\mathrm{CR}$ & $\pm \mathrm{CR}$ & $\mathrm{Li}$ & $\Delta$ & $h r$ & $\Delta h r$ & $f_{\mathrm{x} 14}$ & $L_{\mathrm{x} 27}$ \\
\hline 569 & 11973 & $\lambda$ Ari & 4.79 & 0.28 & F0V & 40.8 & $\mathrm{SB}, \mathrm{VB}$ & 254 & 0.066 & 0.018 & 27 & 45 & -0.50 & 0.20 & 37.2 & 74.2 \\
\hline 575 & 12111 & 48 Cas & 4.54 & 0.16 & A3IV & 35.8 & $\mathrm{SB}, \mathrm{VB}$ & 678 & 0.383 & 0.025 & 677 & 8 & -0.12 & 0.06 & 293.7 & 451.1 \\
\hline 581 & 12230 & 47 Cas & 5.38 & 0.31 & FoVn & 33.6 & $\mathrm{~S}$ & 763 & 2.178 & 0.055 & 999 & 2 & -0.05 & 0.02 & 1752.2 & 2360.7 \\
\hline 582 & 12235 & $112 \mathrm{Psc}$ & 5.88 & 0.62 & G2IV & 31.1 & $\mathrm{~S}$ & 366 & 0.028 & 0.012 & 7 & $1 \overline{5}$ & 0.11 & 0.39 & 24.9 & 28.8 \\
\hline 591 & 12311 & $\alpha \mathrm{Hyi}$ & 2.86 & 0.28 & F0V & 21.9 & $\mathrm{~S}$ & 378 & 0.055 & 0.015 & 21 & 3 & -1.00 & 0.08 & 75.0 & 42.9 \\
\hline 593 & 12363 & $\sigma$ Hyi & 6.16 & 0.44 & F5-6IV-V & 41.3 & $\mathrm{~S}$ & 209 & 0.094 & 0.026 & 21 & 12 & -0.18 & 0.25 & 69.5 & 142.0 \\
\hline 637 & 13445 & & 6.12 & 0.82 & K1V & 10.9 & $\mathrm{~S}$ & 375 & 0.058 & 0.016 & 24 & 27 & -0.63 & 0.18 & 28.9 & 4.1 \\
\hline 638 & 13456 & & 6.01 & 0.39 & F5V & 50.0 & $\mathrm{~S}$ & 373 & 0.035 & 0.012 & 10 & 10 & -0.32 & 0.36 & 22.9 & 68.4 \\
\hline 647 & 13594 & & 6.06 & 0.40 & F4V & 41.5 & VB & 109 & 0.149 & 0.043 & 18 & 44 & -0.17 & 0.27 & 110.1 & 227.4 \\
\hline 650 & 13612 & 66 Cet & 5.54 & 0.57 & F8V & 46.1 & VB & 345 & 0.031 & 0.012 & 8 & 29 & -0.46 & 0.31 & 18.1 & 45.9 \\
\hline 656 & 13871 & $20 \mathrm{Ari}$ & 5.79 & 0.44 & F6IV-V & 40.1 & $\mathrm{~S}$ & 218 & 0.030 & 0.013 & 11 & 7 & -1.00 & 0.34 & 40.6 & 78.1 \\
\hline 657 & 13872 & $21 \mathrm{Ari}$ & 5.58 & 0.49 & F6V & 48.1 & VB & 239 & 0.076 & 0.022 & 21 & 10 & -0.18 & 0.25 & 56.1 & 155.7 \\
\hline 660 & 13974 & $\delta \operatorname{Tri}$ & 4.87 & 0.61 & G0.5V & 10.8 & $\mathrm{SB}$ & 299 & 0.423 & 0.039 & 285 & 5 & -0.38 & 0.09 & 266.6 & 37.5 \\
\hline 692 & 14691 & & 5.46 & 0.35 & FOV & 29.9 & $\mathrm{~S}$ & 323 & 0.036 & 0.013 & 12 & 12 & -0.52 & 0.27 & 19.8 & 21.2 \\
\hline 695 & 14802 & $\kappa$ For & 5.20 & 0.60 & GOVa & 21.9 & $\mathrm{~S}$ & 266 & 0.869 & 0.060 & 726 & 9 & -0.07 & 0.06 & 690.1 & 397.1 \\
\hline 707 & 15089 & $\iota$ Cas & 4.52 & 0.12 & A5pSr & 43.4 & VB & 626 & 0.228 & 0.020 & 338 & 6 & -0.20 & 0.08 & 165.1 & 372.1 \\
\hline 710 & 15144 & & 5.83 & 0.15 & $\mathrm{~A} 6 \mathrm{VpSrCr}$ & 65.6 & $\mathrm{SB}, \mathrm{VB}$ & 358 & 0.092 & 0.020 & 29 & 18 & 0.12 & 0.19 & 82.0 & 422.6 \\
\hline 728 & 15524 & & 5.92 & 0.41 & F6IV & 51.0 & $\mathrm{~S}$ & 228 & 0.142 & 0.028 & 62 & 8 & 0.53 & 0.18 & 157.8 & 491.9 \\
\hline 740 & 15798 & $\sigma$ Cet & 4.75 & 0.45 & F4IV & 25.8 & $\mathrm{~S}$ & 352 & 0.025 & 0.011 & 9 & 34 & -1.00 & 0.24 & 33.9 & 27.0 \\
\hline 741 & 15814 & 29 Ari & 6.04 & 0.54 & $\mathrm{~F} 8 \mathrm{~V}$ & 28.7 & SB & 122 & 0.306 & 0.055 & 58 & 15 & -0.39 & 0.16 & 191.3 & 188.6 \\
\hline 753 & 16160 & & 5.82 & 0.98 & K3V & 7.2 & $\mathrm{SB}, \mathrm{VB}$ & 232 & 0.058 & 0.019 & 19 & 14 & -0.65 & 0.20 & 28.4 & 1.8 \\
\hline 755 & 16170 & & 6.24 & 0.52 & F6V & 53.4 & $\mathrm{~S}$ & 577 & 0.033 & 0.010 & 13 & 9 & -0.31 & 0.25 & 22.1 & 75.6 \\
\hline 756 & 16176 & & 5.90 & 0.48 & F5V & 54.3 & $\mathrm{~S}$ & 304 & 0.032 & 0.012 & 12 & 21 & -0.26 & 0.35 & 22.5 & 79.2 \\
\hline 764 & 16232 & 30 Ari & 7.09 & 0.50 & $\mathrm{~F} 4 \mathrm{~V}$ & 39.4 & VB & 243 & 0.472 & 0.046 & 292 & 8 & -0.02 & 0.09 & 387.6 & 721.0 \\
\hline 772 & 16417 & $\lambda^{2}$ For & 5.79 & 0.66 & G5IV & 25.5 & $\mathrm{~S}$ & 448 & 0.028 & 0.010 & 12 & 5 & -1.00 & 0.20 & 37.8 & 29.5 \\
\hline 780 & 16589 & & 6.49 & 0.52 & G0IV & 53.4 & SB & 522 & 0.081 & 0.015 & 57 & 4 & -0.05 & 0.18 & 64.8 & 221.6 \\
\hline 781 & 16620 & $\epsilon$ Cet & 4.84 & 0.45 & $\mathrm{~F} 5 \mathrm{~V}+\mathrm{F} 6 \mathrm{~V}$ & 27.0 & $\mathrm{SB}, \mathrm{VB}$ & 256 & 0.053 & 0.018 & 13 & 9 & -0.44 & 0.28 & 31.4 & 27.5 \\
\hline 784 & 16673 & & 5.78 & 0.52 & F6V & 21.5 & $\mathrm{~S}$ & 225 & 0.156 & 0.030 & 54 & 13 & -0.80 & 0.10 & 63.5 & 35.2 \\
\hline 788 & 16739 & 12 Per & 4.91 & 0.59 & F9V & 24.7 & $\mathrm{SB}, \mathrm{VB}$ & 558 & 0.034 & 0.010 & 15 & 22 & -0.57 & 0.26 & 17.8 & 13.0 \\
\hline 789 & 16754 & & 4.75 & 0.06 & $\mathrm{~A} 2 \mathrm{~V}$ & 39.8 & $\mathrm{VB}$ & 421 & 0.473 & 0.035 & 450 & 3 & -0.16 & 0.07 & 353.0 & 667.8 \\
\hline 790 & 16765 & $84 \mathrm{Cet}$ & 5.71 & 0.52 & F7IV & 21.6 & VB & 149 & 0.467 & 0.059 & 196 & 2 & -0.12 & 0.12 & 358.3 & 200.5 \\
\hline 799 & 16895 & $\theta$ Per & 4.12 & 0.49 & $\mathrm{~F} 8 \mathrm{~V}$ & 11.2 & VB & 527 & 0.152 & 0.019 & 140 & 4 & -0.69 & 0.08 & 70.9 & 10.7 \\
\hline 802 & 16920 & $\zeta$ Hor & 5.21 & 0.40 & F4IV & 48.8 & $\mathrm{SB}$ & 404 & 0.130 & 0.020 & 76 & 9 & 0.03 & 0.15 & 109.7 & 312.5 \\
\hline 803 & 16955 & & 6.35 & 0.08 & A2Vp: & 107.9 & VB & 424 & 0.021 & 0.010 & 9 & 17 & 0.11 & 0.46 & 18.9 & 262.5 \\
\hline 804 & 16970 & $\gamma$ Cet & 3.47 & 0.09 & $\mathrm{~A} 3 \mathrm{~V}$ & 25.1 & VB & 224 & 0.376 & 0.044 & 159 & 14 & -0.11 & 0.11 & 290.8 & 219.9 \\
\hline 810 & 17051 & $\iota$ Hor & 5.41 & 0.56 & G0V & 17.2 & $\mathrm{~S}$ & 825 & 0.281 & 0.045 & 70 & 7 & -0.28 & 0.14 & 192.0 & 68.3 \\
\hline$* 813$ & 17094 & $\mu$ Cet & 4.27 & 0.31 & FoIV & 25.8 & $\mathrm{SB}, \mathrm{VB}$ & 1263 & 0.101 & 0.009 & 295 & 22 & -0.64 & 0.07 & 49.7 & 39.7 \\
\hline 815 & 17138 & & 6.18 & 0.18 & $\mathrm{~A} 3 \mathrm{~V}$ & 62.5 & $\mathrm{SB}$ & 461 & 0.670 & 0.040 & 910 & 4 & 0.10 & 0.05 & 592.3 & 2771.5 \\
\hline 817 & 17168 & & 6.22 & 0.04 & $\mathrm{~A} 1 \mathrm{~V}$ & 107.1 & SB & 410 & 0.044 & 0.013 & 20 & 11 & 0.17 & 0.28 & 40.4 & 554.6 \\
\hline 818 & 17206 & $\tau^{1}$ Eri & 4.47 & 0.48 & F6V & 14.0 & $\mathrm{SB}$ & 288 & 0.906 & 0.059 & 559 & 11 & -0.22 & 0.06 & 647.5 & 151.3 \\
\hline 820 & 17240 & & 6.30 & 0.00 & A9V & 47.1 & VB & 565 & 0.035 & 0.009 & 24 & 24 & -0.05 & 0.26 & 28.0 & 74.2 \\
\hline 826 & 17390 & & 6.49 & 0.38 & F3IV & 45.1 & $\mathrm{~S}$ & 253 & 0.063 & 0.019 & 22 & 15 & -0.01 & 0.27 & 51.8 & 125.9 \\
\hline 827 & 17438 & & 6.47 & 0.39 & $\mathrm{~F} 2$ & 38.2 & $\mathrm{~S}$ & 283 & 0.293 & 0.035 & 115 & 19 & -0.26 & 0.11 & 202.9 & 354.2 \\
\hline 853 & 17864 & & 6.36 & 0.05 & $\mathrm{~A} 0 \mathrm{~V}$ & 99.8 & $\mathrm{~S}$ & 490 & 0.130 & 0.020 & 88 & 13 & 0.00 & 0.13 & 107.7 & 1283.4 \\
\hline 855 & 17904 & 20 Per & 5.33 & 0.41 & F4IV & 72.1 & $\mathrm{SB}, \mathrm{VB}$ & 488 & 0.028 & 0.009 & 13 & 21 & -1.00 & 0.25 & 38.0 & 236.2 \\
\hline 857 & 17925 & & 6.04 & 0.87 & $\mathrm{~K} 2 \mathrm{~V}$ & 10.4 & $\mathrm{~S}$ & 220 & 1.200 & 0.077 & 883 & 8 & -0.11 & 0.06 & 927.2 & 119.6 \\
\hline 860 & 17948 & & 5.59 & 0.45 & F4V & 26.5 & $\mathrm{~S}$ & 550 & 0.028 & 0.009 & 18 & 30 & -1.00 & 0.18 & 38.5 & 32.3 \\
\hline 863 & 18149 & $\psi$ For & 5.92 & 0.44 & F6IV & 59.4 & $\mathrm{~S}$ & 286 & 0.139 & 0.025 & 57 & 13 & -0.23 & 0.16 & 98.6 & 416.6 \\
\hline 869 & 18256 & $\rho^{3}$ Ari & 5.63 & 0.43 & F6V & 35.4 & $\mathrm{SB}$ & 439 & 0.052 & 0.013 & 25 & 14 & -0.36 & 0.23 & 33.0 & 49.5 \\
\hline 878 & 18404 & 47 Ari & 5.80 & 0.41 & F5IV & 31.8 & $\mathrm{~S}$ & 213 & 0.119 & 0.027 & 32 & 12 & -0.72 & 0.16 & 53.6 & 65.0 \\
\hline 884 & 18466 & & 6.29 & 0.47 & A2-3V:+G: & 144.5 & $\mathrm{SB}$ & 405 & 0.125 & 0.020 & 102 & 5 & 0.37 & 0.14 & 128.5 & 3210.3 \\
\hline 901 & 18692 & $\zeta$ For & 5.71 & 0.40 & $\mathrm{~F} 2 \mathrm{~V}$ & 32.3 & $\mathrm{~S}$ & 288 & 0.192 & 0.031 & 40 & 71 & -0.65 & 0.14 & 93.5 & 117.0 \\
\hline 913 & 18894 & & 6.19 & 0.60 & G0IV- & 32.5 & SB & 81 & 0.141 & 0.051 & 9 & 63 & -0.26 & 0.31 & 97.6 & 123.0 \\
\hline 916 & 18928 & & 6.36 & 0.00 & FOV & 50.0 & $\mathrm{~S}$ & 540 & 0.017 & 0.007 & 9 & 4 & -0.70 & 0.21 & 7.9 & 23.5 \\
\hline 937 & 19373 & $\iota$ Per & 4.05 & 0.59 & G0V & 10.5 & $\mathrm{~S}$ & 523 & 0.021 & 0.007 & 13 & 55 & -1.00 & 0.48 & 29.1 & 3.9 \\
\hline 943 & 19545 & & 6.19 & 0.16 & A5V & 57.6 & $\mathrm{~S}$ & 231 & 0.042 & 0.017 & 13 & 20 & -0.02 & 0.40 & 34.7 & 137.9 \\
\hline 963 & 20010 & $\alpha$ For & 3.8 & 0.52 & F8 & 14.1 & $\mathrm{~V}$ & 212 & 2.792 & 0.168 & 916 & 5 & -0.08 & 0.05 & 2201.8 & 524.6 \\
\hline 971 & 20149 & & 5.52 & 0.01 & $\mathrm{~A} 1 \mathrm{~V} \mathrm{~s}$ & 133.0 & $\mathrm{SI}$ & 466 & 0.037 & 0.010 & 38 & 13 & 0.74 & 0.23 & 45.1 & 954.9 \\
\hline 975 & 20193 & & 6.31 & 0.37 & F4V vw & 62.0 & VB & 460 & 0.035 & 0.010 & 22 & 8 & -0.27 & 0.29 & 23.9 & 110.1 \\
\hline 976 & 20210 & & 6.2 & 0.28 & $\mathrm{~A} 1 \mathrm{~m}$ & 66.8 & $\mathrm{SB}$, & 45 & 0.050 & 0.012 & 27 & 6 & -0.06 & 0.23 & 40.4 & 215.8 \\
\hline 978 & 20277 & & 6.06 & 0.99 & G8IV & 168.9 & $\mathrm{~S}$ & 464 & 0.588 & 0.036 & 845 & 12 & 0.23 & 0.06 & 560.1 & 19121.5 \\
\hline 988 & 20395 & 14 Eri & 6.14 & 0.40 & F1V & 34.7 & $\mathrm{~S}$ & 195 & 0.063 & 0.022 & 15 & 5 & -0.55 & 0.25 & 33.8 & 48.7 \\
\hline 996 & 20630 & $\kappa^{1}$ Cet & 4.83 & 0.68 & G5V & 9.2 & $\mathrm{~S}$ & 286 & 1.201 & 0.095 & 472 & 11 & -0.36 & 0.07 & 768.9 & 77.2 \\
\hline$* 997$ & 20631 & & 5.71 & 0.37 & FOIV & 36.6 & $\mathrm{SB}, \mathrm{VB}$ & 1802 & 0.094 & 0.008 & 392 & 11 & -0.39 & 0.07 & 58.9 & 94.3 \\
\hline 1001 & 20675 & & 5.93 & 0.43 & F6V & 45.7 & $\mathrm{~S}$ & 507 & 0.046 & 0.011 & 36 & 3 & -0.26 & 0.24 & 32.0 & 80.0 \\
\hline 1006 & 20766 & $\zeta^{1} \operatorname{Ret}$ & 5.54 & 0.64 & $\mathrm{G} 2.5 \mathrm{VFe} 1 \mathrm{H} \delta$ & 12.1 & $\mathrm{~S}$ & 328 & 0.095 & 0.020 & 35 & 16 & -0.91 & 0.09 & 33.1 & 5.8 \\
\hline 1008 & 20794 & & 4.27 & 0.71 & G8V & 6.1 & $\mathrm{~S}$ & 519 & 0.026 & 0 & 10 & 16 & -0.76 & 0.30 & 11.1 & 0.5 \\
\hline 1019 & 20995 & & 5.61 & -0.03 & AOV & 161.6 & $\mathrm{~V}$ & 47 & 0.028 & 0.009 & 17 & 26 & 0.19 & 0.33 & 26.0 & 811.7 \\
\hline 1039 & 21379 & & 6.28 & -0.02 & $\mathrm{~A} 0 \mathrm{~V} \mathrm{~s}$ & 104.4 & SB & 419 & 0.030 & 0.011 & 13 & 4 & -0.14 & 0.32 & 23.0 & 299.9 \\
\hline 1042 & 21423 & ${ }^{1}$ For & 6.39 & 0.08 & A1IV & 99.9 & $\mathrm{~S}$ & 134 & 0.175 & 0.039 & 38 & 81 & 0.65 & 0.17 & 205.4 & 2452.1 \\
\hline 1077 & 21903 & & 6.46 & 0.39 & F3V & 56.0 & VB & 576 & 0.061 & 0.012 & 63 & 13 & -0.23 & 0.17 & 43.5 & 163.3 \\
\hline 1083 & 22001 & $\kappa$ Ret & 4.72 & 0.40 & F5IV- & 21.4 & VB & 356 & 0.071 & 0.017 & 32 & 16 & -0.50 & 0.19 & 40.0 & 22.0 \\
\hline 1084 & 22049 & $\epsilon$ Eri & 3.7 & 0.88 & $\mathrm{~K} 2 \mathrm{~V}$ & 3.2 & $\mathrm{VB}$ & 37 & 2.822 & 0.233 & 327 & 3 & -0.44 & 0.07 & 1687.0 & 20.9 \\
\hline 1089 & 22211 & & 6.49 & 0.63 & G0 & 134.6 & $\mathrm{~S}$ & 343 & 0.096 & 0.018 & 68 & 14 & 0.78 & 0.15 & 119.5 & 2589.1 \\
\hline 109 & 22468 & & 5.7 & 0. & G9V & 29.0 & $\mathrm{SB}, \mathrm{VB}$ & 422 & 18.970 & 0.965 & 999 & 2 & -0.07 & 0.05 & 15060.3 & 15121.0 \\
\hline 1107 & 22701 & & 5. & 0.37 & F5IV & 40.9 & $\mathrm{~S}$ & 596 & 0.107 & 0.015 & 122 & 2 & 0.24 & 0.13 & 103.0 & 206.3 \\
\hline 1120 & 22905 & & 6.33 & 0.88 & G5 & 197.6 & $\mathrm{~S}$ & 341 & 0.037 & 0.013 & 13 & 12 & -0.21 & 0.29 & 26.9 & 1257.8 \\
\hline 1136 & 23249 & $\delta$ Eri & 3.54 & 0.9 & $\mathrm{~K} 0+\mathrm{IV}$ & 9.0 & $\mathrm{~S}$ & 532 & 0.019 & 0.008 & 9 & 24 & -0.66 & 0.29 & 9.2 & 0.9 \\
\hline 1174 & 23793 & $30 \mathrm{Tau}$ & 5.07 & -0.13 & B3V+ & 173.3 & $\mathrm{Vl}$ & 366 & 0.048 & 0.013 & 34 & 14 & 0.42 & 0.26 & 50.4 & 1809.8 \\
\hline 1189 & 24071 & & 5.40 & 0.01 & $\mathrm{~A} 1 \mathrm{~V}$ & 49.4 & VB & 70 & 0.099 & 0.044 & 10 & 90 & -0.69 & 0.65 & 46.1 & 134.7 \\
\hline
\end{tabular}


Table 2. continued

\begin{tabular}{|c|c|c|c|c|c|c|c|c|c|c|c|c|c|c|c|c|}
\hline $\mathrm{HR}$ & HD & name & $V$ & $B-V$ & MK type & dist & binary & Exp. & $\mathrm{CR}$ & $\pm \mathrm{CR}$ & $\mathrm{Li}$ & $\Delta$ & $h r$ & $\Delta h r$ & $f_{\mathrm{x} 14}$ & $L_{\mathrm{x} 27}$ \\
\hline 1196 & 24164 & & 6.34 & 0.30 & A $5 \mathrm{~m}$ & 60.1 & $\mathrm{~S}$ & 495 & 0.041 & 0.011 & 21 & 10 & -0.07 & 0.26 & 32.6 & 141.0 \\
\hline 1201 & 24357 & & 5.97 & 0.34 & $\mathrm{~F} 4 \mathrm{~V}$ & 41.4 & $\mathrm{~S}$ & 328 & 0.056 & 0.015 & 30 & 7 & -0.25 & 0.23 & 39.2 & 80.5 \\
\hline 1210 & 24546 & 43 Per & 5.28 & 0.41 & F5IV & 40.8 & SB & 616 & 0.025 & 0.008 & 13 & 12 & -1.00 & 0.18 & 34.7 & 69.1 \\
\hline 1211 & 24554 & 32 Eri & 6.14 & 0.09 & $\mathrm{~A} 2 \mathrm{~V}$ & 105.7 & VB & 352 & 0.207 & 0.025 & 169 & 1 & 0.10 & 0.12 & 183.2 & 2448.8 \\
\hline 1218 & 24740 & $32 \mathrm{Tau}$ & 5.63 & 0.30 & F2IV & 44.8 & $\mathrm{~S}$ & 325 & 0.045 & 0.013 & 23 & 10 & -0.27 & 0.28 & 31.2 & 75.1 \\
\hline 1220 & 24760 & $\epsilon$ Per & 2.89 & -0.18 & $\mathrm{~B} 0.5 \mathrm{~V}+\mathrm{A} 2 \mathrm{~V}$ & 165.0 & $\mathrm{SB}, \mathrm{VB}$ & 514 & 0.069 & 0.013 & 46 & 17 & -0.10 & 0.18 & 53.4 & 1741.3 \\
\hline 1233 & 25102 & & 6.37 & 0.42 & F5V & 39.3 & $\mathrm{~S}$ & 323 & 0.111 & 0.021 & 48 & 5 & -0.30 & 0.17 & 74.8 & 138.5 \\
\hline 1238 & 25202 & & 5.89 & 0.32 & $\mathrm{~F} 4 \mathrm{~V}$ & 45.5 & SB & 307 & 0.023 & 0.010 & 7 & 34 & -1.00 & 0.59 & 31.0 & 76.8 \\
\hline 1245 & 25346 & & 6.05 & 0.44 & F2IV & 61.7 & $\mathrm{~S}$ & 815 & 0.101 & 0.013 & 128 & 9 & -0.02 & 0.11 & 82.9 & 377.8 \\
\hline 1249 & 25457 & & 5.38 & 0.50 & F5V & 19.2 & $\mathrm{~S}$ & 320 & 1.524 & 0.100 & 774 & 10 & -0.03 & 0.06 & 1242.2 & 549.6 \\
\hline 1254 & 25570 & & 5.46 & 0.37 & $\mathrm{~F} 2 \mathrm{~V}$ & 36.0 & $\mathrm{~S}$ & 312 & 0.155 & 0.024 & 90 & 8 & -0.23 & 0.14 & 110.3 & 170.7 \\
\hline 1257 & 25621 & & 5.36 & 0.50 & F6IV & 34.6 & $\mathrm{~S}$ & 323 & 0.239 & 0.030 & 197 & 12 & 0.26 & 0.11 & 231.7 & 332.7 \\
\hline 1262 & 25680 & 39 Tau & 5.90 & 0.62 & G5V & 16.7 & $\mathrm{~S}$ & 178 & 0.424 & 0.051 & 174 & 8 & -0.26 & 0.11 & 293.8 & 98.3 \\
\hline 1269 & 25867 & $\psi \mathrm{Tau}$ & 5.23 & 0.34 & F1V & 27.6 & $\mathrm{~S}$ & 421 & 0.041 & 0.012 & 22 & 6 & -0.17 & 0.27 & 30.2 & 27.5 \\
\hline 1278 & 25998 & 50 Per & 5.51 & 0.46 & $\mathrm{~F} 7 \mathrm{~V}$ & 21.3 & $\mathrm{~S}$ & 473 & 0.843 & 0.044 & 999 & 11 & -0.14 & 0.05 & 638.4 & 347.7 \\
\hline 1279 & 26015 & & 6.01 & 0.40 & F3V & 47.0 & $\mathrm{SB}, \mathrm{VB}$ & 252 & 0.033 & 0.014 & 8 & 16 & -0.17 & 0.40 & 24.6 & 65.1 \\
\hline 1282 & 26101 & & 6.32 & 1.18 & K0 & 118.6 & $\mathrm{~S}$ & 517 & 0.015 & 0.007 & 7 & 53 & -1.00 & 0.56 & 20.7 & 348.3 \\
\hline 1287 & 26322 & $44 \mathrm{Tau}$ & 5.41 & 0.34 & F2IV-V & 59.8 & $\mathrm{~S}$ & 430 & 0.053 & 0.013 & 24 & 21 & 0.14 & 0.24 & 47.9 & 204.9 \\
\hline 1309 & 26690 & $46 \mathrm{Tau}$ & 5.29 & 0.36 & $\mathrm{~F} 2 \mathrm{~V}+\mathrm{F} 5 \mathrm{~V}$ & 37.0 & $\mathrm{SB}, \mathrm{VB}$ & 259 & 0.102 & 0.023 & 55 & 11 & -0.60 & 0.15 & 52.5 & 86.0 \\
\hline 1314 & 26764 & & 5.19 & 0.05 & $\mathrm{~A} 2 \mathrm{Vn}$ & 108.2 & SB & 527 & 0.032 & 0.009 & 21 & 6 & 0.51 & 0.34 & 35.6 & 498.5 \\
\hline 1319 & 26911 & $48 \mathrm{Tau}$ & 6.32 & 0.40 & F5V & 44.4 & $\mathrm{~S}$ & 322 & 0.045 & 0.013 & 19 & 28 & -0.52 & 0.25 & 24.8 & 58.6 \\
\hline 1321 & 26913 & & 6.93 & 0.70 & G5IV & 20.9 & $\mathrm{~S}$ & 271 & 0.502 & 0.044 & 304 & 32 & -0.43 & 0.08 & 302.9 & 158.2 \\
\hline 1322 & 26923 & & 6.31 & 0.59 & G0IV & 21.2 & $\mathrm{~S}$ & 271 & 0.502 & 0.044 & 304 & 36 & -0.43 & 0.08 & 302.9 & 162.7 \\
\hline 1324 & 26961 & & 4.61 & 0.04 & $\mathrm{~A} 2 \mathrm{~V}$ & 97.6 & SB & 516 & 0.469 & 0.031 & 716 & 4 & 0.19 & 0.06 & 437.2 & 4978.2 \\
\hline 1325 & 26965 & $o^{2}$ Eri & 4.43 & 0.82 & $\mathrm{~K} 1-\mathrm{V}$ & 5.0 & VB & 341 & 0.179 & 0.025 & 93 & 43 & -0.14 & 0.13 & 135.8 & 4.1 \\
\hline 1330 & 27084 & & 5.45 & 0.22 & A7V & 79.4 & $\mathrm{~S}$ & 515 & 0.302 & 0.025 & 364 & 5 & -0.02 & 0.08 & 248.0 & 1872.0 \\
\hline 1331 & 27176 & 51 Tau & 5.65 & 0.28 & F0V & 54.8 & $\mathrm{SB}, \mathrm{VB}$ & 422 & 0.072 & 0.015 & 46 & 3 & 0.11 & 0.20 & 64.0 & 230.0 \\
\hline 1341 & 27309 & $56 \mathrm{Tau}$ & 5.38 & -0.14 & A0pSi & 96.9 & $\mathrm{~S}$ & 414 & 0.161 & 0.022 & 100 & 15 & -0.22 & 0.12 & 114.7 & 1288.9 \\
\hline 1342 & 27322 & & 5.88 & 0.00 & A3V & 95.0 & $\mathrm{~S}$ & 523 & 0.023 & 0.008 & 11 & 16 & 0.94 & 0.42 & 3 & 332.7 \\
\hline 1351 & 27397 & $57 \mathrm{Tau}$ & 5.59 & 0.28 & F0IV & 44.8 & $\mathrm{SB}, \mathrm{VB}$ & 416 & 0.093 & 0.017 & 63 & 10 & -0.16 & 0 & 4 & 166.8 \\
\hline 1354 & 27429 & & 6.12 & 0.37 & F3:V & 47.3 & SB & 447 & 0.094 & 0.016 & 60 & 19 & -0.52 & 0 . & 52.4 & 140.6 \\
\hline 1355 & 27442 & $\epsilon$ Ret & 4.44 & 1.08 & K2IVa & 18.2 & VB & 1131 & 0.013 & 0.005 & 12 & 19 & -1.00 & 0.28 & 17.3 & 6.9 \\
\hline 1358 & 27483 & & 6.17 & 0.46 & F6V & 45.9 & SB & 412 & 0.131 & 0.020 & 87 & 4 & -0.50 & 0.12 & 73.9 & 186.1 \\
\hline 1362 & 27536 & & 6.27 & 0.91 & G8IV & 63.2 & $\mathrm{~S}$ & 295 & 0.273 & 0.033 & 164 & 3 & 0.16 & 0.11 & 249.6 & 1191.9 \\
\hline 1368 & 27628 & $60 \mathrm{Tau}$ & 5.72 & 0.32 & A $3 m$ & 45.7 & SB & 431 & 0.031 & 0.011 & 11 & 16 & -0.77 & 0.36 & 13.0 & 32.6 \\
\hline 1374 & 27710 & & 6.01 & 0.35 & F0IV-V & 54.3 & VB & 413 & 0.130 & 0.020 & 93 & 39 & -0.15 & 0.14 & 97.5 & 344.7 \\
\hline 1376 & 27749 & $63 \mathrm{Tau}$ & 5.64 & 0.30 & $\mathrm{~A} 1 \mathrm{~m}$ & 47.2 & SB & 457 & 0.022 & 0.009 & 7 & 9 & -0.34 & 0.35 & 14.4 & 38.6 \\
\hline 1379 & 27786 & 56 Per & 5.76 & 0.40 & $\mathrm{~F} 4 \mathrm{~V}$ & 41.7 & VB & 463 & 0.173 & 0.021 & 145 & 8 & -0.36 & 0.11 & 110.5 & 229.5 \\
\hline 1380 & 27819 & $\delta^{2} \mathrm{Tau}$ & 4.80 & 0.15 & A7V & 44.7 & $\mathrm{SB}$ & 506 & 0.031 & 0.009 & 26 & 80 & 0.97 & 0.26 & 42.2 & 101.1 \\
\hline 1385 & 27901 & & 5.98 & 0.37 & F4V & 49.0 & SB & 568 & 0.061 & 0.012 & 55 & 2 & -0.19 & 0.17 & 44.3 & 127.4 \\
\hline 1389 & 27962 & $\delta^{3} \mathrm{Tau}$ & 4.29 & 0.05 & A2IV & 45.4 & $\mathrm{SB}, \mathrm{VB}$ & 545 & 0.044 & 0.011 & 27 & 31 & -0.66 & 0.16 & 21.0 & 51.7 \\
\hline 1391 & 27991 & $70 \mathrm{Tau}$ & 6.46 & 0.49 & F7V & 46.6 & $\mathrm{SB}$, & 534 & 0.215 & 0.022 & 247 & 13 & -0.34 & 0.09 & 139.8 & 362.8 \\
\hline 1394 & 28052 & $71 \mathrm{Tau}$ & 4.49 & 0.25 & F0V & 47.9 & $\mathrm{SB}, \mathrm{VB}$ & 559 & 0.925 & 0.041 & 999 & 20 & -0.05 & 0.04 & 744.0 & 2045.7 \\
\hline 1404 & 28246 & & 6.39 & 0.44 & F6V & 37.0 & $\mathrm{~S}$ & 826 & 0.123 & 0.014 & 115 & 30 & -0.17 & 0.10 & 91.1 & 149.4 \\
\hline 1405 & 28255 & & 6.29 & 0.66 & $\mathrm{G} 4 \mathrm{~V}$ & 27.1 & VB & 544 & 0.051 & 0.012 & 23 & 18 & -0.88 & 0.20 & 18.7 & 16.4 \\
\hline 1406 & 28271 & & 6.40 & 0.52 & F7V & 54.3 & $\mathrm{SB}$, & 451 & 0.347 & 0.029 & 372 & 4 & 0.17 & 0.08 & 320.0 & 128.4 \\
\hline 1408 & 28294 & $76 \mathrm{Tau}$ & 5.90 & 0.32 & F0IV & 54.3 & $\mathrm{~S}$ & 584 & 0.022 & 0.007 & 13 & 30 & -0.66 & 0.25 & 10.5 & 37.0 \\
\hline 1422 & 28485 & 80 Tau & 5.58 & 0.32 & F0V & 43.6 & $\mathrm{SB}, \mathrm{VB}$ & 541 & 0.056 & 0.012 & 41 & 18 & -0.39 & 0.18 & 35.1 & 79.8 \\
\hline 1430 & 28556 & $83 \mathrm{Tau}$ & 5.40 & 0.26 & F0V & 45.8 & $\mathrm{~S}$ & 589 & 0.069 & 0.012 & 62 & 8 & -0.20 & 0.16 & 49.9 & 125.1 \\
\hline 1432 & 28677 & $85 \mathrm{Tau}$ & 6.02 & 0.34 & $\mathrm{~F} 4 \mathrm{~V}$ & 44.9 & SB & 453 & 0.033 & 0.010 & 18 & 33 & -0.46 & 0.24 & 19.2 & 46.4 \\
\hline 1436 & 28736 & & 6.39 & 0.42 & F5V & 43.2 & $\mathrm{~S}$ & 530 & 0.064 & 0.013 & 39 & 21 & -0.19 & 0. & 46.6 & 104.2 \\
\hline 1438 & 28763 & & 6.21 & 0.12 & A3V & 122.5 & VB & 169 & 0.055 & 0.022 & 11 & 17 & -0.55 & 0.30 & 29.6 & 532.2 \\
\hline 1458 & 29140 & $88 \mathrm{Tau}$ & 4.25 & 0.18 & $\mathrm{~A} 5 \mathrm{~m}$ & 46.1 & SB & 439 & 0.333 & 0.030 & 310 & 10 & -0.16 & 0.08 & 248.5 & 632.5 \\
\hline 1459 & 29169 & & 6.02 & 0.38 & F5IV & 44.2 & $\mathrm{~S}$ & 455 & 0.035 & 0.012 & 10 & 17 & -0.70 & 0.21 & 16.0 & 37.4 \\
\hline 1466 & 29316 & 2 Cam & 5.35 & 0.32 & A8V & 85.0 & VB & 500 & 0.077 & 0.014 & 56 & 13 & -0.08 & 0.19 & 60.6 & 524.6 \\
\hline 1468 & 29329 & & 6.49 & 0.51 & F7V & 32.2 & $\mathrm{~S}$ & 477 & 0.729 & 0.040 & 999 & 4 & -0.01 & 0.05 & 602.3 & 748.4 \\
\hline 1470 & 29364 & & 6.47 & 0.00 & F2V: & 70.0 & $\mathrm{SB}, \mathrm{VB}$ & 457 & 0.054 & 0.013 & 28 & 9 & 0.00 & 0.22 & 45.3 & 265.4 \\
\hline 1472 & 29375 & 89 Tau & 5.79 & 0.31 & FoV & 45.5 & $\mathrm{~S}$ & 424 & 0.018 & 0.008 & 9 & 12 & -0.45 & 0.34 & 10.4 & 25.9 \\
\hline 1473 & 29388 & 90 Tau & 4.27 & 0.12 & A6V & 45.9 & SB & 385 & 0.025 & 0.010 & 11 & 6 & -0.20 & 0.36 & 18.3 & 46.0 \\
\hline 1474 & 29391 & 51 Eri & 5.23 & 0.28 & F0V & 29.8 & $\mathrm{~S}$ & 509 & 1.594 & 0.155 & 275 & 77 & -0.09 & 0.09 & 1248.6 & 1323.2 \\
\hline 1490 & 29646 & & 5.78 & -0.03 & $\mathrm{~A} 2 \mathrm{~V}$ & 102.7 & VB & 460 & 0.099 & 0.016 & 88 & 52 & -0.09 & 0.15 & 77.3 & 975.0 \\
\hline 1501 & 29867 & & 6.45 & 0.00 & A8V & 72.0 & $\mathrm{~S}$ & 457 & 0.030 & 0.010 & 16 & 6 & -0.08 & 0.30 & 23.3 & 144.8 \\
\hline 1502 & 29875 & $\alpha \mathrm{Cae}$ & 4.45 & 0.34 & $\mathrm{~F} 2 \mathrm{~V}$ & 20.1 & VB & 329 & 0.521 & 0.042 & 393 & 7 & -0.32 & 0.07 & 344.3 & 167.0 \\
\hline 1503 & 29992 & $\beta \mathrm{Cae}$ & 5.05 & 0.37 & F1V & 27.7 & $\mathrm{~S}$ & 268 & 0.099 & 0.022 & 39 & 8 & -0.16 & 0.19 & 73.7 & 67.5 \\
\hline 1511 & 30121 & 4 Cam & 5.30 & 0.25 & A3m & 49.7 & $\mathrm{~S}$ & 479 & 0.024 & 0.009 & 10 & 13 & -0.50 & 0.29 & 13.8 & 40.6 \\
\hline 1532 & 30495 & 58 Eri & 5.51 & 0.63 & $\mathrm{G} 2.5 \mathrm{~V}$ & 13.3 & $\mathrm{~S}$ & 539 & 0.490 & 0.046 & 272 & 3 & -0.33 & 0.08 & 321.6 & 68.2 \\
\hline 1543 & 30652 & $\pi^{3}$ Ori & 3.19 & 0.45 & F6V & 8.0 & SB & 392 & 1.957 & 0.147 & 481 & 9 & -0.23 & 0.07 & 1387.7 & 106.9 \\
\hline 1557 & 30985 & & 6.07 & 0.37 & F2-3V & 52.3 & VB & 252 & 0.035 & 0.014 & 11 & 29 & 0.59 & 0.34 & 39.6 & 129.6 \\
\hline 1563 & 31203 & $\iota \mathrm{Pic}$ & 5.61 & 0.33 & F0IV & 37.1 & VB & 937 & 0.107 & 0.019 & 50 & 21 & -0.16 & 0.15 & 79.8 & 131.3 \\
\hline 1564 & 31204 & $\iota \mathrm{Pic}$ & 6.42 & 0.00 & F4V: & 32.1 & VB & 937 & 0.107 & 0.019 & 50 & 20 & -0.16 & 0.15 & 79.8 & 98.3 \\
\hline 1568 & 31278 & 7 Cam & 4.47 & -0.02 & A1V & 115.2 & $\mathrm{SB}, \mathrm{V}$ & 450 & 0.143 & 0.020 & 125 & 36 & 0.17 & 0.13 & 131.5 & 2088.7 \\
\hline 1575 & 31362 & & 6.37 & 0.33 & F0 & 43.2 & $\mathrm{~S}$ & 445 & 0.071 & 0.014 & 48 & 9 & 0.07 & 0.20 & 61.9 & 138.2 \\
\hline 1591 & 31623 & & 6.23 & 0.42 & F2 & 84.0 & $\mathrm{~S}$ & 390 & 0.017 & 0.009 & 7 & 33 & -0.51 & 0.33 & 9.6 & 81.3 \\
\hline 1592 & 31647 & $\omega$ Aur & 4.94 & 0.04 & A1V & 48.8 & VB & 468 & 0.658 & 0.039 & 999 & 5 & 0.10 & 0.05 & 581.8 & 1656.2 \\
\hline 1593 & 31662 & & 6.03 & 0.41 & F4V & 36.9 & VB & 439 & 0.091 & 0.016 & 70 & 3 & -0.24 & 0.17 & 64.3 & 104.7 \\
\hline 1594 & 31675 & & 6.19 & 0.48 & dF6 & 27.9 & $\mathrm{~S}$ & 428 & 0.289 & 0.027 & 296 & 6 & -0.21 & 0.09 & 208.2 & 194.6 \\
\hline 1597 & 31746 & & 6.12 & 0.44 & F3V & 30.9 & $\mathrm{~S}$ & 326 & 0.141 & 0.023 & 83 & 2 & -0.10 & 0.16 & 109.7 & 125.2 \\
\hline 1599 & 31761 & 5 Aur & 5.95 & 0.41 & F5V & 53.0 & VB & 466 & 0.027 & 0.009 & 14 & 38 & -0.26 & 0.32 & 18.9 & 63.7 \\
\hline 1608 & 32008 & 63 Eri & 5.38 & 0.80 & G4V & 54.7 & S & 401 & 0.531 & 0.038 & 513 & 3 & -0.35 & 0.06 & 342.8 & 1225.9 \\
\hline 1614 & 32147 & & 6.22 & 1.06 & K3V & 8.8 & SB & 387 & 0.032 & 0.012 & 9 & 13 & -0.63 & 0.38 & 16.0 & 1.5 \\
\hline
\end{tabular}


Table 2. continued

\begin{tabular}{|c|c|c|c|c|c|c|c|c|c|c|c|c|c|c|c|c|}
\hline $\mathrm{HR}$ & HD & name & $V$ & $B-V$ & MK type & dist & binary & Exp. & $\mathrm{CR}$ & $\pm \mathrm{CR}$ & $\mathrm{Li}$ & $\Delta$ & $h r$ & $\Delta h r$ & $f_{\mathrm{x} 14}$ & $L_{\mathrm{x} 27}$ \\
\hline 1637 & 32537 & 9 Aur & 5.00 & 0.33 & FoV & 26.2 & SB & 450 & 0.051 & 0.013 & 22 & 20 & -0.83 & 0.16 & 19.8 & $\overline{16.3}$ \\
\hline 1639 & 32608 & & 6.52 & 0.16 & A5V & 82.4 & $\mathrm{~S}$ & 465 & 0.028 & 0.009 & 17 & 18 & -0.16 & 0.30 & 21.0 & 170.2 \\
\hline 1647 & 32715 & & 6.41 & 0.40 & F6V & 39.6 & $\mathrm{~S}$ & 415 & 0.070 & 0.015 & 50 & 8 & -0.35 & 0.18 & 45.2 & 84.7 \\
\hline 1649 & 32743 & $\eta^{1} \mathrm{Pic}$ & 5.38 & 0.42 & $\mathrm{~F} 2 \mathrm{~V}$ & 26.2 & VB & 244 & 0.179 & 0.030 & 60 & 7 & -0.22 & 0.15 & 127.9 & 104.9 \\
\hline 1657 & 32964 & 66 Eri & 5.12 & -0.06 & $\mathrm{~B} 9 \mathrm{~V}+\mathrm{A} 1 \mathrm{~V}$ & 85.8 & $\mathrm{SB}, \mathrm{VB}$ & 416 & 0.085 & 0.016 & 65 & 3 & 0.25 & 0.19 & 82.3 & 725.3 \\
\hline 1664 & 33054 & 14 Ori & 5.34 & 0.33 & $\mathrm{Am}$ & 59.4 & VB & 424 & 0.038 & 0.012 & 15 & 15 & -0.71 & 0.17 & 17.4 & 73.5 \\
\hline 1668 & 33167 & & 5.68 & 0.42 & F5V & 46.2 & $\mathrm{~S}$ & 434 & 0.118 & 0.017 & 107 & 7 & 0.09 & 0.14 & 103.5 & 264.5 \\
\hline 1670 & 33204 & & 6.01 & 0.27 & $\mathrm{~A} 5 \mathrm{~m}$ & 54.7 & VB & 452 & 0.033 & 0.010 & 24 & 20 & 0.18 & 0.29 & 30.7 & 109.8 \\
\hline 1673 & 33256 & 68 Eri & 5.12 & 0.44 & $\mathrm{~F} 2 \mathrm{~V}$ & 25.0 & $\mathrm{~S}$ & 406 & 0.021 & 0.009 & 8 & 47 & -1.00 & 0.25 & 29.0 & 21.7 \\
\hline 1674 & 33262 & $\zeta$ Dor & 4.72 & 0.52 & F7V & 11.7 & $\mathrm{~S}$ & 1236 & 0.484 & 0.110 & 26 & 14 & -0.32 & 0.18 & 320.4 & 52.0 \\
\hline 1686 & 33564 & & 5.05 & 0.47 & F6V & 21.0 & VB & 519 & 0.020 & 0.007 & 11 & 10 & -1.00 & 0.40 & 26.8 & 14.1 \\
\hline 1687 & 33608 & & 5.90 & 0.46 & F5V & 38.4 & $\mathrm{~S}$ & 406 & 0.105 & 0.018 & 70 & 12 & -0.14 & 0.16 & 79.8 & 140.9 \\
\hline 1700 & 33875 & & 6.27 & -0.01 & A1V & 129.9 & $\mathrm{~S}$ & 594 & 0.020 & 0.007 & 13 & 9 & 0.14 & 0.37 & 17.7 & 358.0 \\
\hline 1706 & 33959 & 14 Aur & 5.02 & 0.23 & A9IV $\delta$ Del & 82.4 & $\mathrm{SB}, \mathrm{VB}$ & 461 & 5.051 & 0.107 & 999 & 10 & -0.96 & 0.00 & 627.4 & 13211.6 \\
\hline 1717 & 34180 & & 6.15 & 0.39 & FOIV & 45.9 & $\mathrm{~S}$ & 412 & 0.056 & 0.014 & 28 & 52 & -0.11 & 0.23 & 43.2 & 109.0 \\
\hline 1734 & 34499 & 18 Aur & 6.49 & 0.24 & $\mathrm{~A} 7 \mathrm{~V}$ & 72.2 & VB & 456 & 0.032 & 0.010 & 22 & 17 & -0.53 & 0.24 & 17.6 & 109.8 \\
\hline 1747 & 34721 & & 5.96 & 0.58 & G0V & 24.9 & $\mathrm{SB}, \mathrm{VB}$ & 417 & 0.028 & 0.010 & 14 & 47 & -0.03 & 0.37 & 22.9 & 17.0 \\
\hline 1780 & 35296 & $111 \mathrm{Tau}$ & 4.99 & 0.53 & F8V & 14.7 & SB & 443 & 1.395 & 0.057 & 999 & 7 & -0.12 & 0.04 & 070.5 & 275.5 \\
\hline 1782 & 35317 & & 6.11 & 0.50 & F7V & 58.0 & $\mathrm{SB}$, & 424 & 0.058 & 0.014 & 23 & 24 & -0.37 & 0.21 & 37.1 & 149.4 \\
\hline 1785 & 35386 & & 6.49 & 0.50 & $\mathrm{~F} 6 \mathrm{~V}$ & 52.4 & $\mathrm{~S}$ & 444 & 0.101 & 0.019 & 56 & 7 & -0.16 & 0.16 & 75.4 & 247.7 \\
\hline 1817 & 35850 & & 6.35 & 0.51 & $\mathrm{dF} 7$ & 26.8 & $\mathrm{~S}$ & 416 & 2.732 & 0.195 & 675 & 3 & 0.24 & 0.06 & 617.8 & 2256.0 \\
\hline 1857 & 36570 & $19 \mathrm{Cam}$ & 6.15 & 0.01 & A0V & 114.0 & VB & 400 & 0.049 & 0.013 & 26 & 28 & 0.08 & 0.25 & 42.9 & 667.1 \\
\hline 1872 & 36777 & 38 Ori & 5.36 & 0.05 & $\mathrm{~A} 2 \mathrm{~V}$ & 105.8 & VB & 460 & 0.032 & 0.010 & 17 & 23 & 0.06 & 0.31 & 27.2 & 364.1 \\
\hline 1925 & 37394 & & 6.23 & 0.84 & $\mathrm{~K} 1 \mathrm{~V}$ & 12.2 & $\mathrm{~S}$ & 367 & 0.369 & 0.033 & 331 & 16 & -0.55 & 0.07 & 199.0 & 35.7 \\
\hline 1926 & 37430 & $\nu^{1} \mathrm{Col}$ & 6.16 & 0.34 & FOIV & 44.6 & $\mathrm{~S}$ & 520 & 0.115 & 0.017 & 98 & 20 & -0.21 & 0.13 & 83.0 & 197.2 \\
\hline 1935 & 37495 & $\nu^{2} \mathrm{Col}$ & 5.31 & 0.46 & $\mathrm{~F} 4 \mathrm{~V}$ & 42.5 & $\mathrm{~S}$ & 526 & 0.125 & 0.017 & 109 & 9 & -0.14 & 0.13 & 94.3 & 203.6 \\
\hline 1936 & 37501 & & 6.32 & 0.85 & G5IV & 106.7 & $\mathrm{~S}$ & 1090 & 0.036 & 0.007 & 39 & 11 & 1.00 & 0.53 & 48.6 & 662.1 \\
\hline 1940 & 37594 & & 6.00 & 0.27 & $\mathrm{~A} 8 \mathrm{~V} \mathrm{~s}$ & 41.4 & $\mathrm{~S}$ & 466 & 0.017 & 0.008 & 7 & 12 & -0.51 & 0.33 & 9.4 & 19.2 \\
\hline 1967 & 38089 & & 6.02 & 0.40 & F3V & 50.2 & $\mathrm{SB}, \mathrm{VB}$ & 456 & 0.052 & 0.012 & 31 & 9 & -0.66 & 0.19 & 24.8 & 74.7 \\
\hline 1981 & 38385 & & 6.25 & 0.37 & F3V & 53.4 & $\mathrm{~S}$ & 627 & 0.014 & 0.006 & 10 & 14 & -0.86 & 0.15 & 5.1 & 17.4 \\
\hline 1982 & 38392 & & 6.15 & 0.94 & $\mathrm{~K} 2 \mathrm{~V}$ & 9.0 & $\mathrm{C}$ & 488 & 0.343 & 0.029 & 330 & 9 & -0.52 & 0.06 & 190.5 & 18.5 \\
\hline 1988 & 38529 & & 5.95 & 0.78 & $\mathrm{G} 4 \mathrm{~V}$ & 42.4 & 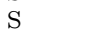 & 481 & 0.069 & 0.013 & 55 & 9 & -0.23 & 0.18 & 48.6 & 104.8 \\
\hline 2007 & 38858 & & 5.97 & 0.64 & $\mathrm{G} 4 \mathrm{~V}$ & 15.6 & $\mathrm{~S}$ & 500 & 0.023 & 0.008 & 10 & 45 & -0.77 & 0.22 & 9.6 & 2.8 \\
\hline 2047 & 39587 & $\chi^{1}$ Ori & 4.41 & 0.59 & G0V & 8.7 & & 431 & 1.942 & 0.068 & 999 & 8 & -0.27 & 0.03 & 1335.9 & 120.0 \\
\hline 2072 & 39937 & & 5.94 & 0.66 & F7IV & 127.2 & $\mathrm{~S}$ & 1659 & 0.655 & 0.020 & 999 & 2 & 0.15 & 0.03 & 596.2 & 546.0 \\
\hline 2094 & 40292 & & 5.29 & 0.31 & F0V $\delta$ Del & 34.9 & $\mathrm{~S}$ & 1218 & 0.009 & 0.004 & 8 & 49 & -0.94 & 0.15 & 3.0 & 4.3 \\
\hline 2118 & 40745 & & 6.22 & 0.36 & F0 & 59.9 & $\mathrm{~S}$ & 528 & 0.018 & 0.008 & 9 & 34 & -0.62 & 0.29 & 9.2 & 39.7 \\
\hline 2124 & 40932 & $\mu$ Ori & 4.12 & 0.16 & $\mathrm{~A} 2 \mathrm{~V}$ & 46.5 & $\mathrm{SB}, \mathrm{VB}$ & 449 & 0.091 & 0.016 & 72 & 4 & -0.13 & 0.15 & 69.6 & 180.3 \\
\hline 2150 & 41547 & & 5.87 & 0.37 & $\mathrm{dF} 4$ & 59.0 & $\mathrm{SB}$ & 542 & 0.034 & 0.009 & 21 & 18 & -0.54 & 0.22 & 18.4 & 76.6 \\
\hline 2157 & 41700 & & 6.35 & 0.52 & G0IV-V & 26.7 & $\mathrm{~S}$ & 933 & 0.636 & 0.028 & 889 & 76 & -0.08 & 0.05 & 501.5 & 427.6 \\
\hline 2162 & 41824 & & 6.58 & 0.72 & G6V & 29.7 & VB & 1042 & 1.902 & 0. & 183 & 5 & 0.13 & 0.10 & 1711.6 & 1809.6 \\
\hline 2174 & 42111 & & 5.73 & 0.07 & $\mathrm{~A} 3 \mathrm{Vn}$ & 186.9 & VB & 478 & 0.083 & 0.015 & 73 & 12 & 0.25 & 0.17 & 80.2 & 3350.9 \\
\hline 2179 & 42278 & & 6.17 & 0.34 & F3IV w & 61.0 & $\mathrm{~S}$ & 515 & 0.025 & 09 & 14 & 8 & -0.08 & 0.31 & 20.0 & 89.2 \\
\hline 2180 & 42301 & & 5.50 & -0.01 & A0IV & 78.3 & $\mathrm{~S}$ & 590 & 0.023 & 0.008 & 14 & 17 & 0.50 & 0.32 & 25.3 & 185.8 \\
\hline 2181 & 42303 & $\pi^{2} \mathrm{Col}$ & 5.50 & 0.00 & $\mathrm{~A} 0 \mathrm{~V}$ & 80.1 & VB & 871 & 0.024 & 0.007 & 12 & 57 & 0.28 & 0.27 & 24.0 & 184.3 \\
\hline 2186 & 42443 & & 5.71 & 0.44 & $\mathrm{~F} 6 \mathrm{~V}$ & 54.1 & $\mathrm{VB}$ & 591 & 0.073 & & 55 & 8 & -0.20 & 0.16 & 52.8 & 184.9 \\
\hline 2208 & 42807 & & 6.45 & 0.67 & $\mathrm{G} 2 \mathrm{~V}$ & 18.1 & $\mathrm{~S}$ & 450 & 0.193 & 0.023 & 172 & 2 & -0.36 & 0.10 & 123.6 & 48.5 \\
\hline 2209 & 42818 & & 4.80 & 0.03 & $\mathrm{~A} 0 \mathrm{Vn}$ & 53.9 & $\mathrm{SB}$ & 359 & 0.047 & 0.014 & 23 & 17 & -0.19 & 0.28 & 34.6 & 120.4 \\
\hline 2217 & 43017 & & 6.92 & 0.45 & F4IV & 48.3 & $\mathrm{VB}$ & 336 & 0.021 & 0.009 & 9 & 78 & -0.65 & 0.42 & 10.2 & 28.5 \\
\hline 2220 & 43042 & 71 Ori & 5.20 & 0.44 & F6V & 21.1 & VB & 403 & 0.140 & 0.020 & 95 & 13 & -0.17 & 0.13 & 103.6 & 55.3 \\
\hline 2225 & 43162 & & 6.39 & 0.72 & G5 & 16.7 & $\mathrm{~S}$ & 651 & 0.655 & 71 & 165 & 21 & -0.39 & 0.09 & 408.7 & 136.3 \\
\hline 2236 & 43358 & & 6 & 0.46 & F5IV: & 92.9 & SB & 479 & 0.021 & 99 & 8 & 12 & -0.12 & 0 & 15.8 & 163.4 \\
\hline 2238 & 43378 & 2 Lyn & 4.48 & 0.01 & $\mathrm{~A} 2 \mathrm{~V} \mathrm{~s}$ & 45.7 & $\mathrm{~S}$ & 366 & 0.019 & & 9 & 22 & -0.16 & 0.44 & 14.0 & 35.1 \\
\hline 2241 & 43386 & 74 Ori & 5.04 & 0.42 & F5IV-V & 19.6 & $\mathrm{~S}$ & 454 & 0.352 & 0.029 & 380 & 3 & -0.23 & 0.07 & 249.5 & 114.8 \\
\hline 2253 & 43683 & & 6.16 & 0.01 & A3V & 195.3 & VB & 443 & 0.021 & 0.009 & 8 & 83 & -1.00 & 0.35 & 28.3 & 1292.0 \\
\hline 2259 & 43821 & & 6.24 & 0.87 & Ko & 106.2 & SB & 476 & 0.042 & 0.012 & 21 & 10 & 0.41 & 0.28 & 44.4 & 599.3 \\
\hline 2261 & 43834 & $\alpha$ Men & 5.09 & 0.72 & G6V & 10.1 & $\mathrm{~S}$ & 1166 & 0.017 & 0.005 & 21 & 16 & -1.00 & 0.23 & 23.1 & 2.9 \\
\hline 2265 & 43940 & & 5.87 & 0.14 & A3V & 62.1 & $\mathrm{~S}$ & 790 & 0.500 & 0.027 & 788 & 5 & -0.05 & 0.05 & 402.6 & 1858.1 \\
\hline 2272 & 44092 & & 6.43 & 0.06 & A1V & 103.7 & $\mathrm{C}$ & 346 & 0.017 & 0.008 & 8 & 23 & 0.28 & 0.59 & 16.9 & 218.1 \\
\hline 2280 & 44333 & & 6.31 & 0.24 & $\mathrm{~A} 4.5 \mathrm{~V}$ & .5 & $\mathrm{VI}$ & 484 & 0.037 & 10 & 20 & 14 & 0.12 & 0 . & 32.7 & 411.2 \\
\hline 2298 & 44769 & $\epsilon$ Mon & 4.44 & 0.18 & A5IV & 39.4 & $\mathrm{SB}, \mathrm{V}$ & 462 & 0.080 & 0.0 & 58 & 10 & -0.48 & 4 & 46.2 & 85.7 \\
\hline 2299 & 44770 & & 6.72 & 0.45 & F5V & 24.3 & VB & 462 & 0.080 & 0.0 & 58 & 4 & -0.48 & 0.14 & 46.2 & 32.7 \\
\hline 2320 & 45229 & $\nu \mathrm{Pic}$ & 5.61 & 0.24 & $\mathrm{Am}$ & 50.3 & $\mathrm{~S}$ & 1432 & 0.018 & 0.004 & 31 & 9 & -0.10 & 0.23 & 14.2 & 42.9 \\
\hline 2328 & 45380 & & 6.27 & -0.04 & AoVn & 125.5 & VB & 523 & 0.137 & 0.018 & 156 & 21 & 0.36 & 0.12 & 140.2 & 2640.5 \\
\hline 2351 & 45638 & & 6.59 & 0.00 & A9IV & 60.8 & $\mathrm{~S}$ & 451 & 0.133 & 0.019 & 97 & 6 & -0.28 & 0.13 & 91.0 & 402.3 \\
\hline 2353 & 45680 & & 6.48 & 0.39 & F3V & 49.8 & VB & 716 & 0.147 & 0.016 & 181 & 5 & -0.11 & 0.10 & 113.6 & 336.7 \\
\hline 2365 & 45947 & & 6.24 & 0.38 & $\mathrm{~F} 2$ & 35.6 & $\mathrm{~S}$ & 466 & 0.055 & 0.012 & 39 & 10 & -0.04 & 0.21 & 44.2 & 66.9 \\
\hline 2384 & 46273 & & 5.27 & 0.41 & $\mathrm{~F} 2 \mathrm{~V}$ & 51.7 & $\mathrm{SB}$ & 870 & 0.070 & 0.010 & 73 & 9 & -0.56 & 0.11 & 37.4 & 119.7 \\
\hline 2386 & 46304 & & 5.60 & 0.25 & FOVn & 43.2 & $\mathrm{VB}$ & 500 & 0.141 & 0.018 & 132 & 9 & -0.39 & 0.11 & 87.8 & 196.3 \\
\hline 2401 & 46588 & & 5.45 & 0.50 & $\mathrm{~F} 8 \mathrm{~V}$ & 17.9 & $\mathrm{~S}$ & 52 & & & 9 & 18 & -1.00 & 0 . & 22.7 & 8.7 \\
\hline 2421 & 47105 & $\gamma$ Gem & 1.93 & & AOIV & 32.1 & SB & 447 & 0.219 & & 182 & 1 & -0.03 & 0.10 & 178.5 & 220.5 \\
\hline 2424 & 47144 & & 5.59 & -0.14 & AOV & 177.9 & VB & 413 & 0.068 & 0.015 & 32 & 13 & 0.06 & 0.22 & 58.3 & 2209.4 \\
\hline 2439 & 47415 & & 6.38 & 0.53 & F8IV & 35.2 & SB & 422 & 0.161 & 0.022 & 133 & 11 & -0.22 & 0.12 & 114.7 & 169.7 \\
\hline 2466 & 48097 & $26 \mathrm{Gem}$ & 5.21 & 0.06 & $\mathrm{~A} 2 \mathrm{~V}$ & 42.7 & SB & 443 & 0.093 & 0.018 & 51 & 9 & -0.40 & 0.16 & 57.3 & 125.0 \\
\hline 2468 & 48189 & & 6.18 & 0.62 & G1-2V & 21.7 & VB & 966 & 2.325 & 0.050 & 999 & 4 & -0.15 & 0.02 & 1747.2 & 981.5 \\
\hline 2481 & 48501 & & 6.13 & 0.34 & F0 & 48.9 & VB & 285 & 0.043 & 0.015 & 11 & 50 & -0.36 & 0.30 & 27.8 & 79.7 \\
\hline 2482 & 48543 & & 6.29 & 0.34 & $\mathrm{~A} 3 \mathrm{~V}+\mathrm{F} / \mathrm{G}$ & 91.3 & VB & 611 & 0.047 & 0.011 & 35 & 2 & -0.53 & 0.18 & 26.0 & 259.1 \\
\hline 2483 & 48682 & $\psi^{5}$ Aur & 5.25 & 0.56 & G0V & 16.5 & VB & 337 & 0.048 & 0.014 & 17 & 43 & -0.18 & 0.29 & 35.1 & 11.4 \\
\hline 2485 & 48767 & & 6.33 & 0.00 & $\mathrm{dF} 6$ & 47.0 & $\mathrm{SB}, \mathrm{V}$ & 350 & 0.116 & 0.020 & 79 & 7 & -0.23 & 0.16 & 82.0 & 217.0 \\
\hline 2486 & 48766 & & 6.28 & 0.47 & dF5 & 47.0 & $\mathrm{VB}$ & 350 & 0.116 & 0.020 & 79 & 12 & -0.23 & 0.16 & 82.0 & 217.0 \\
\hline
\end{tabular}


Table 2. continued

\begin{tabular}{|c|c|c|c|c|c|c|c|c|c|c|c|c|c|c|c|c|}
\hline $\mathrm{HR}$ & $\mathrm{HD}$ & name & V & $B-V$ & MK type & dist & binary & Exp. & $\mathrm{CR}$ & $\pm \mathrm{CR}$ & $\mathrm{Li}$ & $\Delta$ & $h r$ & $\Delta h r$ & $f_{\mathrm{x} 14}$ & $L_{\times 27}$ \\
\hline 2491 & 48915 & $\alpha \mathrm{CMa}$ & -1.46 & 0.00 & $\mathrm{~A} 1 \mathrm{Vm}$ & 2.6 & $\mathrm{SB}, \mathrm{VB}$ & 272 & 12.730 & 0.219 & 999 & 7 & -0.99 & 0.00 & 3899.2 & 32.4 \\
\hline 2500 & 49095 & & 5.92 & 0.48 & F6V & 24.3 & $\mathrm{~S}$ & 577 & 0.015 & 0.006 & 9 & 30 & -0.65 & 0.31 & 7.1 & 5.0 \\
\hline 2514 & 49434 & & 5.75 & 0.28 & F1V & 40.1 & $\mathrm{~S}$ & 367 & 0.019 & 0.009 & 9 & 39 & -1.00 & 0.30 & 26.4 & 50.7 \\
\hline 2530 & 49933 & & 5.77 & 0.39 & $\mathrm{~F} 2 \mathrm{~V}$ & 29.9 & VB & 224 & 0.366 & 0.043 & 163 & 6 & -0.04 & 0.11 & 296.5 & 317.0 \\
\hline 2539 & 50018 & 59 Aur & 6.12 & 0.37 & $\mathrm{~F} 2 \mathrm{~V} \delta \mathrm{Del}$ & 162.6 & VB & 361 & 0.054 & 0.014 & 28 & 37 & -0.01 & 0.25 & 44.2 & 1397.4 \\
\hline 2548 & 50223 & & 5.14 & 0.45 & F5V & 25.2 & $\mathrm{~S}$ & 606 & 0.016 & 0.006 & 10 & 28 & -0.87 & 0.32 & 5.8 & 4.4 \\
\hline 2550 & 50241 & $\alpha \mathrm{Pic}$ & 3.27 & 0.21 & A7IV & 30.3 & $\mathrm{~S}$ & 1213 & 0.078 & 0.009 & 138 & 8 & -0.13 & 0.11 & 59.2 & 65.2 \\
\hline 2551 & 50277 & & 5.77 & 0.26 & FOVn & 62.6 & $\mathrm{~S}$ & 325 & 0.057 & 0.016 & 22 & 17 & -0.13 & 0.27 & 43.8 & 205.3 \\
\hline 2552 & 50282 & & 6.30 & 0.99 & K0 & 146.2 & $\mathrm{~S}$ & 291 & 0.088 & 0.019 & 41 & 28 & 0.61 & 0.17 & 101.3 & 2591.7 \\
\hline 2564 & 50635 & $38 \mathrm{Gem}$ & 4.65 & 0.30 & FoVp & 27.9 & $\mathrm{SB}, \mathrm{VB}$ & 344 & 0.263 & 0.031 & 196 & 3 & -0.22 & 0.10 & 188.2 & 175.8 \\
\hline 2607 & 51733 & & 5.46 & 0.36 & $\mathrm{dF0}$ & 38.6 & $\mathrm{VB}$ & 500 & 0.095 & 0.015 & 80 & 16 & -0.35 & 0.14 & 61.6 & 109.6 \\
\hline 2612 & 51825 & & 6.23 & 0.46 & F8IV-V & 43.2 & VB & 432 & 0.412 & 0.032 & 462 & 5 & -0.02 & 0.07 & 338.0 & 754.6 \\
\hline 2626 & 52362 & & 6.22 & 0.00 & $\mathrm{~A} 0 \mathrm{~V}$ & 130.2 & $\mathrm{SB}$ & 550 & 0.015 & 0.007 & 10 & 13 & -0.42 & 0.35 & 9.4 & 191.3 \\
\hline 2637 & 52619 & & 6.27 & 0.45 & F5V & 68.2 & $\mathrm{~S}$ & 427 & 0.027 & 0.010 & 10 & 2 & -0.37 & 0.33 & 17.5 & 97.1 \\
\hline 2658 & 53253 & & 6.43 & -0.04 & A0V & 125.2 & $\mathrm{~S}$ & 535 & 0.023 & 0.008 & 13 & 49 & -0.14 & 0.33 & 17.6 & 329.1 \\
\hline 2677 & 53952 & & 6.14 & 0.37 & $\mathrm{~F} 2 \mathrm{~V}$ & 55.7 & VB & 482 & 0.015 & 0.007 & 7 & 67 & -0.44 & 0.38 & 9.3 & 34.4 \\
\hline 2683 & 54118 & & 5.17 & -0.04 & $\mathrm{~A} 0 \mathrm{pSi}$ & 86.6 & $\mathrm{~S}$ & 541 & 0.023 & 0.009 & 10 & 86 & 0.14 & 0.34 & 20.8 & 186.7 \\
\hline 2705 & 54958 & & 6.23 & 0.40 & Fo & 49.8 & VB & 391 & 0.052 & 0.013 & 27 & 10 & -0.14 & 0.25 & 39.1 & 116.1 \\
\hline 2720 & 55568 & & 6.10 & 0.27 & $\mathrm{~A} 8 \mathrm{~V}$ & 47.6 & $\mathrm{~S}$ & 424 & 0.051 & 0.014 & 22 & 34 & -0.54 & 0.19 & 27.6 & 74.6 \\
\hline 2735 & 55864 & $\gamma^{1} \mathrm{Vol}$ & 5.69 & 0.40 & $\mathrm{~F} 2 \mathrm{~V}$ & 45.1 & $\mathrm{SB}, \mathrm{VB}$ & 777 & 0.079 & 0.012 & 46 & 34 & -0.28 & 0.13 & 54.1 & 131.7 \\
\hline 2740 & 55892 & & 4.49 & 0.32 & FoIV & 21.2 & $\mathrm{~S}$ & 176 & 0.106 & 0.027 & 34 & 22 & -0.58 & 0.18 & 55.6 & 29.8 \\
\hline 2776 & 56963 & & 5.77 & 0.32 & $\mathrm{~A} 7 \mathrm{~s}$ & 34.5 & $\mathrm{~S}$ & 377 & 0.035 & 0.012 & 15 & 22 & -0.63 & 0.20 & 17.4 & 24.8 \\
\hline 2777 & 56986 & $\delta \mathrm{Gem}$ & 3.53 & 0.34 & F2IV & 18.0 & $\mathrm{SB}, \mathrm{V}$ & 443 & 0.108 & 0.019 & 57 & 21 & -0.56 & 0.12 & 57.8 & 22.5 \\
\hline 2788 & 57167 & & 5.70 & 0.35 & F1V & 44.0 & SB & 395 & 0.443 & 0.034 & 554 & 2 & -0.01 & 0.07 & 366.1 & 849.3 \\
\hline 2813 & 57852 & & 6.05 & 0.42 & F0-2IV-V & 34.8 & VB & 309 & 0.354 & 0.035 & 252 & 12 & -0.14 & 0.10 & 267.7 & 387.5 \\
\hline 2814 & 57853 & & 6.60 & 0.59 & G0V:e & 34.8 & VB & 309 & 0.354 & 0.035 & 252 & 9 & -0.14 & 0.10 & 267.7 & 387.5 \\
\hline 2832 & 58461 & & 5.78 & 0.42 & FOV & 34.8 & $\mathrm{~S}$ & 184 & 0.074 & 0.022 & 27 & 11 & -0.46 & 0.25 & 43.6 & 63.3 \\
\hline 2846 & 58728 & $63 \mathrm{Gem}$ & 5.22 & 0.39 & $\mathrm{~F} 5 \mathrm{~V}+\mathrm{F} 5 \mathrm{~V}$ & 34.0 & SB & 442 & 0.485 & 0.035 & 418 & 7 & -0.35 & 0.06 & 313.1 & 434.0 \\
\hline 2848 & 58805 & & 6.47 & 0.42 & F3V & 39.1 & $\mathrm{~S}$ & 340 & 0.034 & 0.012 & 13 & 21 & -0.45 & 0.32 & 20.1 & 36.8 \\
\hline 2852 & 58946 & $\rho$ Gem & 4.18 & 0.32 & FOV & 18.5 & $\mathrm{SB}$ & 445 & 0.347 & 0.029 & 399 & 2 & -0.29 & 0.08 & 234.9 & 96.2 \\
\hline 2869 & 59466 & & 6.58 & 0.06 & A1IV & 106.0 & $\mathrm{~S}$ & 295 & 0.024 & 0.010 & 15 & 18 & -0.01 & 0.43 & 19.9 & 267.7 \\
\hline 2882 & 59967 & & 6.65 & 0.63 & G4V & 21.8 & $\mathrm{~S}$ & 271 & 0.320 & 0.037 & 195 & 15 & -0.36 & 0.10 & 205.1 & 116.3 \\
\hline 2890 & 60178 & $\alpha$ Gem & 2.88 & 0.04 & $\mathrm{~A} 2 \mathrm{Vm}$ & 15.8 & $\mathrm{SB}, \mathrm{VB}$ & 440 & 3.697 & 0.091 & 999 & 58 & -0.15 & 0.02 & 2778.3 & 830.4 \\
\hline 2891 & 60179 & $\alpha \mathrm{Gem}$ & 1.98 & 0.03 & A1V & 15.8 & $\mathrm{SB}, \mathrm{VB}$ & 440 & 3.697 & 0.091 & 999 & 58 & -0.15 & 0.02 & 2778.3 & 830.4 \\
\hline 2909 & 60584 & & 5.83 & 0.44 & $\mathrm{dF} 4$ & 29.1 & $\mathrm{SB}, \mathrm{VB}$ & 204 & 0.363 & 0.044 & 180 & 6 & -0.25 & 0.11 & 253.9 & 256.6 \\
\hline 2910 & 60585 & & 5.87 & 0.00 & $\mathrm{dF} 6$ & 29.1 & VB & 204 & 0.363 & 0.044 & 180 & 14 & -0.25 & 0.11 & 253.9 & 256.6 \\
\hline 2923 & 60951 & & 6.34 & 0.99 & G5 & 211.9 & SB & 246 & 0.019 & 0.010 & 9 & 4 & 1.00 & 0.80 & 25.3 & 1359.5 \\
\hline 2943 & 61421 & $\alpha \mathrm{CMi}$ & 0.38 & 0.42 & F5IV- & 3.5 & $\mathrm{SB}, \mathrm{V}$ & 400 & 3.655 & 0.098 & 999 & 7 & -0.90 & 0.01 & 1293.9 & 18.9 \\
\hline 2962 & 61859 & & 6.02 & 0.00 & F7V & 54.6 & SB & 434 & 0.028 & 0.011 & 9 & 39 & -0.24 & 0.32 & 19.9 & 71.1 \\
\hline 2997 & 62613 & & 6.56 & 0.73 & G8V & 17.0 & $\mathrm{~S}$ & 551 & 0.021 & 0.008 & 9 & 21 & -0.66 & 0.26 & 9.9 & 3.4 \\
\hline 3015 & 62952 & 4 Pup & 5. & & $\mathrm{~F}($ & 72.5 & $\mathrm{~S}$ & 349 & & & 10 & 56 & 0.19 & & 21.7 & 136.4 \\
\hline 3028 & 63332 & & 6.02 & 0.46 & F6V & 30.0 & $\mathrm{~S}$ & 342 & 0.019 & 0.009 & 9 & 35 & -1.00 & 0.70 & 26.4 & 28.5 \\
\hline 3029 & 63336 & 5 Pup & 5.48 & 0.48 & dF5 & 30.2 & VB & 234 & 0.054 & 0.017 & 16 & 33 & -1.00 & 0.33 & 72.8 & 79.7 \\
\hline 3070 & 64185 & & 5 & 0.42 & F1V & & VB & 711 & 0. & 0.013 & 88 & 20 & -0.35 & & 58.4 & 85.2 \\
\hline 3072 & 64235 & & 5.76 & 0.41 & F5IV & 40.9 & VB & 227 & 0.311 & 0.037 & 191 & 14 & 0.12 & 0.12 & 278.3 & 557.0 \\
\hline 3079 & 64379 & & 5.01 & 0.44 & F5V & 18.0 & VB & 154 & 0.589 & 0.063 & 226 & 5 & -0.20 & 0.10 & 426.9 & 165.9 \\
\hline 3087 & 64685 & & & & F2IV & & $\mathrm{S}$ & 363 & 0.0 & 0.014 & 15 & 15 & -0.25 & & 31.5 & 54.9 \\
\hline 3119 & 65626 & & 6.49 & 0.62 & $\mathrm{~F} 8 \mathrm{~V}$ & 101.6 & SB & 443 & 1.347 & 0.057 & 999 & 5 & 0.03 & 0.04 & 1140.8 & 4096.1 \\
\hline 3131 & 65810 & & 4.61 & & $\mathrm{~A} 2 \mathrm{Vn}$ & 73.0 & $\mathrm{~S}$ & 198 & 0.0 & 0.015 & 10 & 6 & -1.00 & 0.30 & 44.0 & 280.6 \\
\hline 3144 & 66011 & & & & & & $\mathrm{~S}$ & & & & 21 & 13 & -0.24 & & 87.3 & 371.6 \\
\hline 3173 & 67006 & 27 Lyn & 4.84 & 0. & A 2 Y & 66.8 & VB & 408 & 0.026 & 0.010 & 13 & 19 & -0.17 & 0.33 & 19.6 & 104.6 \\
\hline 3191 & 67767 & $\psi \mathrm{Cnc}$ & 5.73 & 0.81 & G7V & 42.0 & S & 366 & 0.181 & 0.023 & 152 & 11 & -0.10 & 0.12 & 141.1 & 297.4 \\
\hline 3208 & 68257 & $\zeta^{1} \mathrm{Cnc}$ & 5.63 & 0.54 & F8V & 25.6 & VB & 372 & 0.129 & 0.020 & 82 & 18 & -0.59 & 0.12 & 67.1 & 52.5 \\
\hline 3209 & 68256 & $\zeta^{1} \mathrm{Cnc}$ & 6.02 & 0.54 & F9V & 25.6 & VB & 372 & 0.129 & 0.020 & 82 & 18 & -0.59 & 0.12 & 67.1 & 52.5 \\
\hline 3210 & 68255 & $\zeta^{2} \mathrm{Cnc}$ & 6.20 & 0.60 & G5V & 25.6 & VB & 372 & 0.129 & 0.020 & 82 & 13 & -0.59 & 0.12 & 67.1 & 52.5 \\
\hline 3220 & 68456 & & 4.7 & & F5 & 21.4 & $\mathrm{~S}$ & 366 & 0.351 & 0.032 & 292 & 5 & -0.18 & 0.09 & 258.5 & 141.5 \\
\hline 3221 & 68457 & & 6.4 & 0. & $\mathrm{~A} 7 \mathrm{Vm}$ & 153.1 & VB & 420 & & 0.0 & 7 & 26 & -0.41 & 0.31 & 12.2 & 340.9 \\
\hline 3254 & 69548 & 30 Lyn & 5.8 & 0.3 & F4V & 31.7 & $\mathrm{~S}$ & 282 & 0.110 & 0.022 & 49 & 17 & -0.42 & 8 & 66.7 & 80.0 \\
\hline 3259 & 69830 & & 5.9 & & $\mathrm{G} 7.5 \mathrm{~V}$ & 12.6 & $\mathrm{~S}$ & 355 & & 0.011 & 13 & 1 & -0.65 & 0.29 & 15.7 & 3.0 \\
\hline 3260 & 69863 & & 5 & & A2 & 74 & $\mathrm{VI}$ & 398 & 0.0 & 0.0 & 75 & 16 & 0.04 & 0. & 81.2 & 535.5 \\
\hline 3274 & 70267 & & 6.4 & & F5 & 77.9 & $\mathrm{Vl}$ & 517 & & 0.011 & 24 & 12 & -0.33 & 0 . & 26.9 & 195.2 \\
\hline 3276 & 70302 & & 6.1 & & Ko & 218.3 & $\mathrm{VI}$ & 3 & 0.050 & 0.014 & 24 & 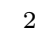 & 0.73 & 0. & 60.9 & 3473.3 \\
\hline 3297 & 70958 & $1 \mathrm{Hya}$ & 5.6 & 0 & F3V & & SB & 307 & 0.686 & 0.050 & 548 & 10 & 0.01 & 0.07 & 574.0 & 508.5 \\
\hline 3313 & 71153 & $24 \mathrm{Cnc}$ & 7.8 & & & & VB & & & & 74 & 22 & -0.10 & & .2 & 1321.3 \\
\hline 3316 & 71196 & & 6.0 & & F3 & 33 & $\mathrm{~s}$ & 3 & 0.1 & 0. & 93 & 5 & -0.07 & 0.14 & 31.7 & 181.6 \\
\hline 3337 & 71663 & & 6 & 0 & A5 & & SB & 295 & 0.1 & 0.0 & 89 & 14 & 0.03 & 0.16 & 134.8 & 1172.4 \\
\hline 3352 & 71973 & & & & & & SB, & & & & 39 & 4 & -0.18 & & 39.0 & 328.3 \\
\hline 3365 & 72291 & 32 Lyn & & & F5 Vb & 37 & $\mathrm{~S}$ & 30 & 0.0 & 9 & 34 & 15 & -0.55 & 0. & 46.5 & 77.5 \\
\hline 3385 & 72688 & & 6.36 & 0.95 & K0V & 130.7 & $\mathrm{~S}$ & 406 & 0.715 & 0.063 & 392 & 7 & 0.26 & 0.08 & 692.3 & 14153.5 \\
\hline 3391 & 72905 & $\pi^{1} \mathrm{UMa}$ & 5.64 & 0.62 & $\mathrm{G} 1.5 \mathrm{Vb}$ & 14.3 & $\mathrm{~S}$ & 392 & 0.797 & 0.047 & 820 & 4 & -0.32 & 0.05 & 526.9 & 128.4 \\
\hline 3395 & 72945 & & 5.9 & & F8 & & & 3 & & & 49 & 3 & & 0.13 & 65.1 & 54.9 \\
\hline 3396 & 72946 & & 7.25 & 0.7 & G5 & 23.4 & $\mathrm{VE}$ & 333 & 0.118 & 0.021 & 49 & 12 & -0.53 & 0.1 & 65.1 & 42.7 \\
\hline 3401 & 73029 & & 6.4 & & $\mathrm{~A} 2 \mathrm{~V}$ & 110.5 & $\mathrm{~S}$ & 467 & & 0.007 & 8 & 88 & -0.20 & 0.41 & 10.7 & 156.7 \\
\hline 3410 & 73262 & $\delta$ Hya & & & & 54 & $\mathrm{SI}$ & 32 & & 0.021 & 43 & 12 & -0.44 & 0.17 & 60.7 & 218.9 \\
\hline 3430 & 73752 & & 5.0 & 0.73 & G6IV & 19.9 & $\mathrm{SB}, \mathrm{VB}$ & 374 & 0.047 & 0.014 & 17 & 16 & -0.76 & 0.21 & 20.1 & 9.6 \\
\hline 343 & 739 & & & & F11 & & $\mathrm{SB}, \mathrm{VB}$ & & & & 86 & 2 & -0.18 & & 90.7 & 175.6 \\
\hline 3451 & 74243 & & & & F7 & 65 & $\mathrm{~S}$ & 25 & & & 20 & 5 & 0.24 & & 41.6 & 212.3 \\
\hline 3455 & 74341 & & 6.34 & 0.2 & A3V & 69.5 & VB & 491 & 0.531 & 0.050 & 305 & 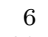 & 0.27 & 0.09 & 517.5 & 2994.4 \\
\hline 3460 & 74405 & $\theta \mathrm{Vol}$ & 5.2 & 0.0 & A0 & 73.3 & $\mathrm{VE}$ & 380 & 0.033 & 0.012 & 9 & 39 & 0.32 & 0.48 & 33.3 & 214.3 \\
\hline 3472 & 74688 & & 6.41 & 0.52 & F2IV & 87.0 & VB & 350 & 0.028 & 0.011 & 15 & 8 & 0.56 & 0.42 & 31.1 & 281.6 \\
\hline
\end{tabular}


Table 2. continued

\begin{tabular}{|c|c|c|c|c|c|c|c|c|c|c|c|c|c|c|c|c|}
\hline $\mathrm{HR}$ & HD & name & $V$ & $B-V$ & MK type & dist & binary & Exp. & $\mathrm{CR}$ & $\pm \mathrm{CR}$ & $\mathrm{Li}$ & $\Delta$ & $h r$ & $\Delta h r$ & $f_{\mathrm{x} 14}$ & $L_{\times 27}$ \\
\hline 3485 & 74956 & $\delta \mathrm{Vel}$ & 1.96 & 0.04 & A1V & 24.4 & VB & 553 & 264 & 0.034 & 109 & 8 & -0.23 & 0.12 & 187.3 & 134.0 \\
\hline 3499 & 75332 & & 6.25 & 0.49 & F7Vn & 28.7 & $\mathrm{~S}$ & 296 & 0.448 & 0.043 & 277 & 4 & 0.00 & 0.09 & 372.0 & 366.3 \\
\hline 3524 & 75747 & & 6.05 & 0.23 & $\mathrm{~A} 5 \mathrm{~V}+\mathrm{A} 7 \mathrm{~V}$ & 97.8 & SB & 675 & 0.314 & 0.033 & 188 & 31 & 0.00 & 0.10 & 261.1 & 2985.0 \\
\hline 3537 & 76143 & & 5.35 & 0.42 & F5IV & 52.5 & VB & 395 & 0.029 & 0.011 & 10 & 12 & -0.12 & 0.35 & 22.3 & 73.7 \\
\hline 3538 & 76151 & & 6.00 & 0.67 & $\mathrm{G} 2 \mathrm{~V}$ & 17.1 & $\mathrm{~S}$ & 307 & 0.118 & 0.023 & 49 & 8 & -0.58 & 0.13 & 61.8 & 21.6 \\
\hline 3569 & 76644 & $\iota \mathrm{UMa}$ & 3.14 & 0.19 & A7IV & 14.6 & $\mathrm{SB}, \mathrm{VB}$ & 285 & 0.169 & 0.027 & 81 & 5 & -0.34 & 0.14 & 110.2 & 28.2 \\
\hline 3570 & 76653 & & 5.71 & 0.48 & $\mathrm{~F} 6 \mathrm{~V}$ & 24.1 & $\mathrm{~S}$ & 440 & 0.461 & 0.034 & 403 & 2 & -0.30 & 0.07 & 309.5 & 215.9 \\
\hline 3579 & 76943 & & 3.97 & 0.44 & F5V & 16.4 & $\mathrm{SB}, \mathrm{VB}$ & 400 & 0.676 & 0.045 & 543 & 5 & -0.46 & 0.05 & 396.9 & 128.2 \\
\hline 3584 & 77084 & & 6.18 & 0.46 & F6V & 39.6 & $\mathrm{~S}$ & 317 & 0.080 & 0.018 & 42 & 12 & -0.09 & 0.21 & 62.4 & 116.7 \\
\hline 3598 & 77370 & & 5.16 & 0.42 & F3V & 26.2 & VB & 355 & 0.219 & 0.028 & 155 & 5 & -0.32 & 0.11 & 144.6 & 118.6 \\
\hline 3616 & 78154 & $\sigma^{2} \mathrm{UMa}$ & 4.80 & 0.49 & F6IV & 20.5 & VB & 438 & 0.059 & 0.014 & 23 & 21 & -0.78 & 0.15 & 24.5 & 12.3 \\
\hline 3625 & 78366 & & 5.93 & 0.60 & F9V & 19.1 & $\mathrm{~S}$ & 411 & 0.334 & 0.030 & 284 & 5 & -0.41 & 0.08 & 204.9 & 89.8 \\
\hline 3626 & 78418 & $75 \mathrm{Cnc}$ & 5.98 & 0.66 & G5IV-V & 31.3 & SB & 365 & 0.090 & 0.018 & 39 & 40 & -0.65 & 0.14 & 43.7 & 51.2 \\
\hline 3635 & 78661 & & 6.48 & 0.34 & $\mathrm{~F} 2 \mathrm{Vp}$ & 37.7 & $\mathrm{~S}$ & 342 & 0.032 & 0.012 & 11 & 34 & -0.72 & 0.24 & 14.6 & 24.8 \\
\hline 3649 & 79066 & & 6.35 & 0.32 & A9IV $\delta$ Del & 50.9 & $\mathrm{~S}$ & 348 & 0.034 & 0.012 & 12 & 8 & -0.36 & 0.32 & 21.7 & 67.2 \\
\hline 3650 & 79096 & $\pi^{1} \mathrm{Cnc}$ & 6.51 & 0.73 & G9V & 20.5 & VB & 189 & 0.061 & 0.022 & 14 & 19 & -1.00 & 0.44 & 83.4 & 41.9 \\
\hline 3690 & 80081 & 38 Lyn & 3.82 & 0.06 & $\mathrm{~A} 3 \mathrm{~V}$ & 37.4 & $\mathrm{SB}, \mathrm{V}$ & 461 & 0.210 & 0.024 & 171 & 6 & -0.11 & 0.10 & 162.2 & 271.2 \\
\hline 3701 & 80441 & & 6.12 & 0.38 & $\mathrm{~F} 2 \mathrm{~V}+\mathrm{F} 4 \mathrm{~V}$ & 47.8 & $\mathrm{VB}$ & 458 & 0.065 & 0.014 & 37 & 3 & -0.04 & 0.20 & 52.6 & 143.8 \\
\hline 3712 & 80671 & & 5.39 & 0.42 & $\mathrm{~F} 4 \mathrm{~V}$ & 33.5 & VB & 297 & 0.049 & 0.015 & 19 & 7 & -0.31 & 0.27 & 32.5 & 43.7 \\
\hline 3714 & 80719 & & 6.33 & 0.46 & $\mathrm{dF} 6$ & 48.3 & SB & 412 & 0.085 & 0.016 & 62 & 7 & 0.19 & 0.18 & 79.5 & 222.1 \\
\hline 3750 & 81809 & & 5.38 & 0.64 & $\mathrm{G} 2 \mathrm{~V}$ & 31.2 & $\mathrm{SB}, \mathrm{VB}$ & 395 & 0.030 & 0.012 & 9 & 22 & -0.89 & 0.26 & 10.7 & 12.5 \\
\hline 3756 & 81919 & & 6.65 & 0.20 & A3 & 114.7 & VB & 628 & 0.064 & 0.012 & 57 & 14 & 0.17 & 0.18 & 59.1 & 930.5 \\
\hline 3757 & 81937 & $23 \mathrm{UMa}$ & 3.67 & 0.33 & FoIV & 23.1 & VB & 346 & 0.042 & 0.013 & 14 & 7 & -1.00 & 0.30 & 57.6 & 36.9 \\
\hline 3759 & 81997 & $\tau^{1}$ Hya & 4.60 & 0.46 & F6V & 17.1 & $\mathrm{~S}$ & 365 & 0.574 & 0.041 & 507 & 18 & -0.25 & 0.06 & 401.0 & 140.3 \\
\hline 3761 & 82068 & & 6.05 & 0.15 & $\mathrm{~A} 3 \mathrm{Vn}$ & 68.3 & $\mathrm{~S}$ & 334 & 0.052 & 0.014 & 23 & 25 & 0.58 & 0.27 & 59.4 & 331.3 \\
\hline 3785 & 82428 & & 6.14 & 0.24 & FoVn & 54.3 & SB & 342 & 0.025 & 0.011 & 8 & 1 & -0.54 & 0.30 & 13.5 & 47.4 \\
\hline 3786 & 82434 & $\psi$ Vel & 3.60 & 0.36 & F3IV+F0IV & 18.6 & VB & 427 & 0.558 & 0.038 & 552 & 4 & -0.33 & 0.06 & 366.2 & 150.9 \\
\hline 3795 & 82554 & $\iota$ Cha & 5.36 & 0.45 & F3-5IV & 56.5 & $\mathrm{~S}$ & 429 & 0.018 & 0.008 & 8 & 55 & -0.47 & 0.41 & 10.6 & 40.5 \\
\hline 3806 & 82685 & & 6.42 & 0.00 & $\mathrm{~F} 2 \mathrm{~V}+\mathrm{F} 1 \mathrm{~V}$ & 69.6 & VB & 480 & 0.062 & 0.013 & 42 & 4 & -0.40 & 0.18 & 38.4 & 222.7 \\
\hline 3811 & 82780 & & 6.76 & 0.35 & $\mathrm{~F} 2 \mathrm{~V}$ & 84.7 & $\mathrm{SB}, \mathrm{VB}$ & 369 & 0.065 & 0.016 & 27 & 9 & -0.34 & 0.19 & 42.5 & 365.2 \\
\hline 3812 & 82785 & & 6.43 & 0.33 & F0IV & 72.7 & VB & 390 & 0.042 & 0.013 & 17 & 24 & -0.11 & 0.28 & 32.4 & 204.9 \\
\hline 3815 & 82885 & $11 \mathrm{LMi}$ & 5.41 & 0.77 & G8V & 11.2 & VB & 467 & 0.298 & 0.027 & 254 & 24 & -0.55 & 0.07 & 160.9 & 24.1 \\
\hline 3828 & 83261 & & 6.53 & 0.38 & $\mathrm{~F} 2$ & 68.3 & VB & 480 & 0.053 & 0.013 & 27 & 2 & -0.58 & 0.17 & 27.7 & 154.4 \\
\hline 3852 & 83808 & $o$ Leo & 3.52 & 0.49 & F6II+ & 41.5 & SB & 342 & 0.072 & 0.017 & 34 & 16 & -0.51 & 0.18 & 40.5 & 83.4 \\
\hline 3857 & 83951 & $13 \mathrm{LMi}$ & 6.14 & 0.36 & F3V & 53.3 & $\mathrm{~S}$ & 471 & 0.026 & 0.009 & 14 & 22 & -0.10 & 0.35 & 20.5 & 69.6 \\
\hline 3893 & 85217 & $4 \mathrm{Sex}$ & 6.24 & 0.48 & F7Vn & 45.9 & SB & 417 & 0.181 & 0.022 & 166 & 11 & -0.15 & 0.12 & 136.2 & 343.4 \\
\hline 3928 & 86146 & $19 \mathrm{LMi}$ & 5.14 & 0.46 & F6V s & 28.9 & SB & 366 & 0.087 & 0.019 & 31 & 6 & -0.66 & 0.15 & 42.0 & 41.9 \\
\hline 3954 & 87141 & & 5.74 & 0.48 & F5V & 47.3 & $\mathrm{~S}$ & 545 & 0.053 & 0.012 & 25 & 16 & -0.17 & 0.22 & 39.5 & 105.6 \\
\hline 3969 & 87500 & & 6.37 & 0.37 & $\mathrm{~F} 2 \mathrm{Vn}$ & 114.0 & $\mathrm{~S}$ & 237 & 0.033 & 0.014 & 9 & 21 & 1.00 & 0.37 & 44.4 & 690.2 \\
\hline 3979 & 87822 & & 6. & & $\mathrm{~F} 4 \mathrm{~V}$ & 63.3 & $\mathrm{VH}$ & & 0.041 & 12 & 16 & 26 & -0.11 & 0.26 & 31.8 & 152.6 \\
\hline 3991 & 88215 & & 5.31 & 0.36 & F5V & 27.3 & SB & 466 & 0.027 & 0.010 & 10 & 10 & -0.78 & 0.17 & 11.3 & 10.1 \\
\hline 4013 & 88742 & & 6.38 & 0.59 & G1V & 22.7 & $\mathrm{~S}$ & 281 & 0.080 & 0.021 & 23 & 35 & -0.14 & 0.24 & 60.8 & 37.6 \\
\hline 4040 & 89169 & & 6. & & F3V & 102.0 & VB & 394 & 0.039 & 0.012 & 17 & 19 & 0.59 & 0.35 & 44.3 & 551.4 \\
\hline 4059 & 89490 & & 6.37 & 0.90 & Ko & 89.8 & S & 429 & 0.136 & 0.020 & 87 & 22 & 0.20 & 0.14 & 127.3 & 1227.7 \\
\hline 4060 & 89565 & & 6.32 & 0.33 & F1IV & 53.3 & $\mathrm{~S}$ & 448 & 0.016 & 0.008 & 8 & 30 & -0.17 & 0.41 & 11.9 & 40.3 \\
\hline 4061 & 89569 & & 5. & 0. & $\mathrm{~F} 6 \mathrm{~V}$ & 35.8 & $\mathrm{~S}$ & 232 & 0.106 & 0.024 & 47 & 16 & 0.03 & 0. & 90.1 & 138.4 \\
\hline 4062 & 89571 & & 5.50 & 0.00 & FOIV & 40.8 & $\mathrm{~S}$ & 533 & 0.031 & 0.010 & 15 & 19 & -0.54 & 0.2 & 16.8 & 33.4 \\
\hline 4065 & 89715 & & 5 & 0.05 & A1V & 89.4 & VB & 251 & 0.067 & 0.018 & 23 & 28 & 0.11 & 0. & 60.0 & 574.6 \\
\hline 4079 & 89995 & & & & Fe & 55.7 & VB & 390 & 0.035 & $0 .($ & 14 & 20 & -0.22 & & 24.8 & 91.9 \\
\hline 4084 & 90089 & & 5.2 & 0.37 & $\mathrm{~F} 2 \mathrm{~V}$ & 21.5 & $\mathrm{~S}$ & 329 & 0.309 & 0.033 & 181 & 4 & -0.21 & 0.10 & 222.5 & 122.9 \\
\hline 4096 & 90470 & & 6 & 0 & $\mathrm{~A} 2 \mathrm{~V}$ & 66.3 & $\mathrm{~S}$ & 470 & 0.034 & 0.011 & 15 & 10 & -0.16 & 0. & 25.0 & 131.3 \\
\hline 4102 & 90589 & & & & F2 & 16.2 & SB & & 0.182 & 17 & 25 & 8 & -0.43 & 0. & 109.8 & 34.5 \\
\hline 4112 & 90839 & $36 \mathrm{UMa}$ & 4.8 & 0.52 & F8V & 12.9 & $\mathrm{~S}$ & 572 & 0.195 & 0.021 & 172 & 5 & -0.67 & 0.07 & 93.0 & 18.4 \\
\hline 4130 & 91280 & & 6.05 & 0. & F7 & 42.4 & $\mathrm{~S}$ & 314 & 0.068 & 0.017 & 29 & 6 & 0.20 & 0. & 63.3 & 136.2 \\
\hline 4141 & 91480 & $37 \mathrm{UMa}$ & 5.16 & & F1 & 26.5 & $\mathrm{~S}$ & & 0.104 & 0.016 & 72 & 13 & 0.11 & & 92.8 & 77.7 \\
\hline 4149 & 91706 & & 6.1 & 0.5 & F7I & 63.5 & $\mathrm{~S}$ & 351 & 0.040 & 0.013 & 16 & 12 & -0.19 & 0. & 29.2 & 140.7 \\
\hline 4155 & 91858 & & 6.57 & 0.2 & F0 & 57.6 & S & 395 & 0.034 & 0.012 & 11 & 62 & -0.28 & 0.35 & 23.0 & 91.2 \\
\hline 4167 & 92139 & & 3.8 & 0. & F4IV+ & 26.5 & $\mathrm{SB}$, & 338 & 0.203 & 0.028 & 138 & 3 & -0.35 & 0.11 & 130.9 & 110.1 \\
\hline 4182 & 92588 & $33 \mathrm{Sex}$ & 6.26 & 0.88 & K1IV & 34.4 & $\mathrm{~S}$ & 386 & 0.167 & 0.024 & 119 & 8 & -0.45 & 0.11 & 98.9 & 140.0 \\
\hline 4203 & 93152 & $42 \mathrm{LMi}$ & 5.24 & -0.0 & A1 & 115.7 & $\mathrm{SE}$ & 408 & 0.024 & 0.010 & 11 & 13 & -0.22 & & 17.4 & 278.2 \\
\hline 4229 & 93742 & $40 \mathrm{Sex}$ & 6.6 & 0.2 & A2IV & 95.9 & $\mathrm{VB}$ & 22 & 0.037 & 0.016 & 11 & 4 & -0.16 & & 27.3 & 300.4 \\
\hline 4251 & 94388 & & 5.24 & 0.47 & $\mathrm{~F} 6 \mathrm{~V}$ & 31.3 & $\mathrm{~S}$ & 289 & 0.070 & 0.018 & 37 & 13 & 0.13 & 0.24 & 63.4 & 74.4 \\
\hline 4263 & 94660 & & 6.11 & -0.0 & $\mathrm{~A} 0 \mathrm{pSi}$ & 152.0 & $\mathrm{~S}$ & & 0.143 & 0.025 & 68 & 1 & 0.14 & 0.17 & 129.4 & 3577.0 \\
\hline 4281 & 95216 & & 6.55 & 0. & F5 & 44.8 & $\mathrm{~S}$ & 272 & 0.063 & 0.018 & 20 & 17 & -0.28 & 0.24 & 42.9 & 102.9 \\
\hline 4314 & 96202 & $\chi^{1}$ Нуа & 4.94 & 0. & F3IV & 43.5 & VB & 289 & 0.032 & 0.013 & 12 & 9 & -1.00 & 0.24 & 43.3 & 98.1 \\
\hline 4345 & 97334 & & 6.41 & 0 & G0 & 21.7 & S & 207 & 0.320 & 0.045 & 114 & 8 & -0.26 & 0.12 & 222.0 & 125.3 \\
\hline 4350 & 97495 & & 5.36 & & & 50.0 & $\mathrm{SE}$ & & & & 25 & 36 & 0.08 & & 68.4 & 204.1 \\
\hline 4363 & 97855 & & 6.5 & & F6 & 39.1 & $\mathrm{~V}$ & 384 & 0.034 & 0.012 & 11 & 85 & -0.69 & 0. & 15.7 & 28.7 \\
\hline 4369 & 98088 & & 6.1 & 0.2 & A8 & 129.0 & $\mathrm{SB}, \mathrm{V}$ & 283 & 0.013 & 0.007 & 8 & 31 & 0.71 & 0.83 & 15.3 & 305.4 \\
\hline 4370 & 98096 & & 6.3 & & F3 & 55.4 & VB & 221 & 0.044 & 0.017 & 10 & 29 & -0.36 & 0.42 & 28.1 & 103.2 \\
\hline 4373 & 98221 & & & & F4I & 46.7 & $\mathrm{~S}$ & 351 & 0.150 & 0.024 & 80 & 19 & -0.33 & 0.13 & 98.4 & 256.6 \\
\hline 4374 & 98230 & $\xi \mathrm{UN}$ & 4.8 & $0 .($ & G0 & & & 192 & 4.539 & 0.157 & 999 & 4 & -0.21 & 0.03 & 3266.7 & \\
\hline 4375 & 98231 & $\xi \mathrm{UN}$ & 4.4 & 0.5 & G0V & & $\mathrm{SB}, \mathrm{VE}$ & 192 & 4.539 & 0.157 & 999 & 4 & -0.21 & 0.03 & 3266.7 & \\
\hline 4380 & 98353 & $55 \mathrm{UMa}$ & 4.7 & 0. & A1 & 56.1 & SB & 25 & 0.053 & 0.016 & 21 & 20 & -0.41 & 0.28 & 32.6 & 122.8 \\
\hline 4384 & 98560 & & 5.99 & 0.4 & F6 & 37.4 & $\mathrm{~S}$ & 429 & 0.051 & 0.013 & 30 & 7 & 0.51 & 0.24 & 56.1 & 93.9 \\
\hline 4399 & 99028 & $\iota$ Leo & 3.94 & 0.4 & F4IV & 24.2 & $\mathrm{SB}, \mathrm{V}$ & 384 & 0.185 & 0.024 & 108 & 14 & -0.65 & 0.09 & 90.0 & 63.3 \\
\hline 440 & 99211 & & 4.08 & 0.2 & A5 & 25.7 & $\mathrm{Vl}$ & 303 & 0.030 & 0.013 & 9 & 28 & -0.54 & 0.35 & 16.6 & 13.1 \\
\hline 4408 & 99285 & $81 \mathrm{Leo}$ & 5.57 & 0.36 & $\mathrm{~F} 2 \mathrm{~V}$ & 47.3 & $\mathrm{VI}$ & 366 & 0.069 & 0.016 & 37 & 12 & -0.15 & 0.22 & 52.2 & 139.7 \\
\hline 4414 & 99491 & 83 Leo & 6.50 & 0.79 & KOIV & 17.7 & VB & 386 & 0.030 & 0.012 & 7 & 44 & -0.98 & 0.21 & 9.3 & 3.5 \\
\hline 4422 & 99787 & $57 \mathrm{UMa}$ & 5.31 & 0.01 & $\mathrm{~A} 2 \mathrm{~V}$ & 64.1 & SB & 290 & 0.054 & 0.017 & 17 & 9 & -0.19 & 0.30 & 39.7 & 195.6 \\
\hline
\end{tabular}


Table 2. continued

\begin{tabular}{|c|c|c|c|c|c|c|c|c|c|c|c|c|c|c|c|c|}
\hline $\mathrm{HR}$ & HD & name & $V$ & $B-V$ & MK type & dist & binary & Exp. & $\mathrm{CR}$ & $\pm \mathrm{CR}$ & $\mathrm{Li}$ & $\Delta$ & $h r$ & $\Delta h r$ & $f_{\mathrm{x} 14}$ & $L_{\mathrm{x} 27}$ \\
\hline 4437 & 100180 & 88 Leo & 6.20 & 0.57 & G0V & 23.0 & VB & 398 & 0.031 & 0.010 & 16 & 12 & -1.00 & 0.46 & 41.6 & 26.4 \\
\hline 4443 & 100286 & & 5.81 & 0.53 & F8V & 26.7 & VB & 354 & 0.035 & 0.013 & 10 & 14 & -0.73 & 0.21 & 15.4 & 13.2 \\
\hline 4444 & 100287 & & 5.64 & 0.53 & F8V & 26.7 & VB & 354 & 0.035 & 0.013 & 10 & 5 & -0.73 & 0.21 & 15.4 & 13.2 \\
\hline 4454 & 100518 & & 6.55 & 0.18 & $\mathrm{~A} 2 \mathrm{~m}$ & 113.6 & S & 419 & 0.055 & 0.014 & 18 & 5 & -0.31 & 0.21 & 36.6 & 565.5 \\
\hline 4455 & 100563 & 89 Leo & 5.77 & 0.46 & F5V & 26.6 & S & 417 & 0.242 & 0.027 & 197 & 2 & -0.33 & 0.09 & 158.7 & 134.0 \\
\hline 4496 & 101501 & $61 \mathrm{UMa}$ & 5.33 & 0.72 & G8V & 9.5 & $\mathrm{~S}$ & 314 & 0.348 & 0.037 & 194 & 13 & -0.75 & 0.07 & 150.9 & 16.4 \\
\hline 4502 & 101615 & & 5.55 & 0.00 & $\mathrm{~A} 0 \mathrm{~V}$ & 64.2 & SB & 155 & 0.091 & 0.028 & 21 & 17 & -0.43 & 0.27 & 54.9 & 270.8 \\
\hline 4505 & 101688 & & 6.59 & 0.00 & F2IV-V & 61.3 & $\mathrm{~S}$ & 438 & 0.059 & 0.014 & 36 & 13 & -0.52 & 0.18 & 32.8 & 147.8 \\
\hline 4528 & 102510 & 4 Vir & 5.32 & 0.02 & $\mathrm{~A} 1$ & 58.9 & S & 434 & 0.019 & 0.008 & 10 & 15 & 0.44 & 0.42 & 20.4 & 84.9 \\
\hline 4533 & 102634 & & 6.15 & 0.52 & F7V & 34.2 & $\mathrm{~S}$ & 392 & 0.025 & 0.010 & 12 & 12 & -0.83 & 0.24 & 10.0 & 13.9 \\
\hline 4535 & 102660 & & 6.04 & 0.27 & A3m & 62.7 & SB & 320 & 0.027 & 0.011 & 15 & 6 & 0.21 & 0.39 & 25.5 & 119.9 \\
\hline 4536 & 102713 & & 5.70 & 0.46 & F5IV & 69.5 & SB & 367 & 0.067 & 0.017 & 29 & 10 & -0.32 & 0.21 & 44.0 & 254.5 \\
\hline 4539 & 102845 & & 6.13 & 0.95 & K0 & 206.2 & S & 323 & 0.130 & 0.022 & 80 & 7 & 0.54 & 0.18 & 145.3 & 7392.9 \\
\hline 4540 & 102870 & $\beta$ Vir & 3.61 & 0.55 & F9V & 10.9 & $\mathrm{~S}$ & 417 & 0.344 & 0.030 & 264 & 8 & -0.63 & 0.07 & 170.9 & 24.3 \\
\hline 4554 & 103287 & $\gamma \mathrm{UMa}$ & 2.44 & 0.00 & $\mathrm{~A} 0 \mathrm{Ve}$ & 25.6 & SB & 401 & 0.050 & 0.014 & 22 & 27 & -0.23 & 0.24 & 35.6 & 28.0 \\
\hline 4599 & 104671 & $\theta^{1} \mathrm{Cru}$ & 4.33 & 0.27 & $\mathrm{Am}$ & 70.5 & $\mathrm{SB}, \mathrm{VB}$ & 369 & 0.029 & 0.011 & 11 & 9 & -0.67 & 0.29 & 13.7 & 81.4 \\
\hline 4600 & 104731 & & 5.15 & 0.41 & F6V & 24.2 & $\mathrm{~S}$ & 173 & 0.067 & 0.023 & 14 & 13 & -0.31 & 0.31 & 44.7 & 31.3 \\
\hline 4606 & 104904 & & 6.27 & 0.59 & F6V & 76.3 & $\mathrm{~S}$ & 786 & 0.121 & 0.014 & 187 & 10 & 0.00 & 0.11 & 100.6 & 700.0 \\
\hline 4627 & 105678 & & 6.35 & 0.50 & F6IV & 72.2 & S & 675 & 0.034 & 0.008 & 30 & 6 & -0.19 & 0.22 & 24.5 & 153.0 \\
\hline 4634 & 105841 & & 6.08 & 0.39 & F2-3IV & 88.8 & $\mathrm{~S}$ & 365 & 0.023 & 0.009 & 9 & 9 & -0.85 & 0.21 & 8.8 & 83.3 \\
\hline 4646 & 106112 & & 5.14 & 0.33 & A5m & 33.7 & SB & 89 & 0.271 & 60 & 46 & 7 & -0.16 & 0.20 & 202.1 & 74.2 \\
\hline 4657 & 106516 & & 6.11 & 0.46 & F5V & 22.6 & S & 386 & 0.042 & 0.012 & 20 & 18 & -0.94 & 0.20 & 13.9 & 8.5 \\
\hline 4686 & 107192 & & 6.28 & 0.35 & F2V & 46.6 & $\mathrm{~S}$ & 909 & 0.125 & 0.013 & 201 & 7 & -0.18 & 0.10 & 91.7 & 237.9 \\
\hline 4698 & 107398 & & 7.13 & 0.36 & $\mathrm{~F} 3 \mathrm{~V}+$ & 92.3 & VB & 449 & 0.029 & 0.010 & 10 & 18 & -0.41 & 0.29 & 17.6 & 179.7 \\
\hline 4708 & 107705 & 17 Vir & 6.40 & 0.60 & $\mathrm{~F} 8 \mathrm{~V}$ & 29.8 & VB & 409 & 0.027 & 0.011 & 8 & 12 & -0.98 & 0.20 & 8.5 & 9.0 \\
\hline 4758 & 108799 & & 6.35 & 0.56 & G0V & 25.0 & VB & 257 & 0.620 & 0.050 & 432 & 3 & -0.18 & 0.08 & 456.1 & 340.6 \\
\hline 4767 & 108954 & & 6.21 & 0.54 & F8-G0V & 21.9 & S & 343 & 0.033 & 0.013 & 8 & 34 & -0.39 & 0.30 & 20.6 & 11.9 \\
\hline 4785 & 109358 & $\beta$ CVn & 4.26 & 0.59 & G0V & 8.4 & SB & 509 & 0.023 & 0.008 & 12 & 44 & -1.00 & 0.35 & 31.8 & 2.7 \\
\hline 4796 & 109573 & & 5.80 & 0.01 & $\mathrm{~A} 0 \mathrm{~V}$ & 67.1 & VB & 284 & 0.113 & 0.024 & 42 & 18 & -0.24 & 0.17 & 79.6 & 28.4 \\
\hline 4825 & 110379 & $\gamma \mathrm{Vir}$ & 3.65 & 0.36 & F0V & 11.8 & $\mathrm{SB}, \mathrm{V}$ & 146 & 2.039 & 0.121 & 888 & 9 & -0.10 & 0.05 & 586.3 & 65.6 \\
\hline 4826 & 110380 & $\gamma \mathrm{Vir}$ & 3.68 & 0.00 & F0V & 11.8 & VB & 146 & 2.039 & 0.121 & 888 & 16 & -0.10 & 0.05 & 1586.3 & 265.6 \\
\hline 4855 & 111164 & $34 \mathrm{Vir}$ & 6.07 & 0.12 & A3V & 74.6 & $\mathrm{~S}$ & 434 & 0.043 & 0.013 & 12 & 14 & 0.28 & 0.27 & 42.3 & 281.5 \\
\hline 4856 & 111199 & & 6.26 & 0.55 & F7V & 66.3 & VB & 394 & 0.039 & 0.014 & 13 & 6 & -0.34 & 0.28 & 25.7 & 135.1 \\
\hline 4864 & 111395 & & 6.31 & 0.70 & G7V & 17.2 & $\mathrm{~S}$ & 424 & 0.133 & 0.021 & 77 & 14 & -0.54 & 0.11 & 72.6 & 25.6 \\
\hline 4867 & 111456 & & 5.85 & 0.46 & F5V & 24.2 & $\mathrm{~S}$ & 479 & 0.503 & 0.034 & 565 & 2 & -0.19 & 0.06 & 367.7 & 256.8 \\
\hline 4891 & 111998 & 38 Vir & 6.11 & 0.50 & F5V & 32.5 & S & 341 & 0.135 & 0.023 & 61 & 15 & -0.40 & 0.14 & 83.8 & 106.0 \\
\hline 4892 & 112014 & & 5.85 & -0.06 & $\mathrm{~A} 0 \mathrm{~V}+\mathrm{A} 2 \mathrm{~V}$ & & $\mathrm{SB}, \mathrm{VB}$ & 713 & 0.013 & 0.005 & 10 & 17 & 0.14 & 0.42 & 11.5 & \\
\hline 4914 & 112412 & $\alpha^{1} \mathrm{CVn}$ & 5.60 & 0.34 & FoV & 33.8 & VB & 500 & 0.076 & 0.015 & 42 & 4 & -0.38 & 0.17 & 48.0 & 65.6 \\
\hline 4915 & 112413 & $\alpha^{2} \mathrm{CVn}$ & 2.90 & -0.12 & $\mathrm{~A} 0 \mathrm{pSi}$ & 33.8 & VB & 500 & 0.076 & 0.015 & 42 & 17 & -0.38 & 0.17 & 48.0 & 65.6 \\
\hline 4922 & 112935 & & 6.02 & 0.38 & F0V & 40.0 & $\mathrm{~S}$ & 262 & 0.127 & 0.025 & 54 & 4 & -0.02 & 0.19 & 103.9 & 199.1 \\
\hline 4926 & 113022 & & 6.20 & 42 & F6V s & 40.8 & $\mathrm{~S}$ & 500 & 0.070 & 0.014 & 40 & 5 & -0 & 0.16 & 6 & 86.9 \\
\hline 4931 & 113139 & $78 \mathrm{UMa}$ & 4.9 & 36 & F2V & 25.0 & VB & 545 & 0.226 & 22 & 206 & 13 & -0 & 0.07 & 11 & 88.1 \\
\hline 4934 & 113337 & & 6.00 & 0.41 & F6V & 37.4 & $\mathrm{~S}$ & 517 & 0.119 & 0.017 & 92 & 14 & -0.04 & 0.14 & 96.5 & 161.8 \\
\hline 4946 & 113848 & 39 Com & 5.99 & 0.39 & F4V & 49.9 & VB & 512 & 0.059 & 0.013 & 29 & 25 & -0.09 & 0.20 & 46.5 & 138.8 \\
\hline 4966 & 114371 & & 5.91 & 0.42 & F3IV-V & 44.1 & $\mathrm{SB}, \mathrm{VB}$ & 386 & 0.018 & 0.008 & 7 & 10 & -0.71 & 0.27 & 8.1 & 18.8 \\
\hline 4968 & 114378 & $\alpha$ Com & 5.22 & 0.45 & F5V & & VB & 244 & 0.876 & 0.063 & 538 & 8 & -0.38 & 0.06 & 551.7 & \\
\hline 4969 & 114379 & $\alpha$ Com & 5.22 & 0.00 & F5V & & VB & 244 & 0.876 & 0.063 & 538 & 8 & -0.38 & 0.06 & 551.7 & \\
\hline 4979 & 114613 & & 4.85 & 0.70 & G3V & 20.5 & $\mathrm{~S}$ & 282 & 0.100 & 0.022 & 34 & 8 & -0.70 & 0.15 & 45.9 & 23.0 \\
\hline 4980 & 114630 & & 6.16 & 60 & G0V & 39.8 & $\mathrm{SB}, \mathrm{V}$ & 141 & 0.757 & 0.075 & 283 & 8 & 0.00 & 0.10 & 628.8 & 1192.3 \\
\hline 4983 & 114710 & $\beta$ Com & 4.2 & 57 & F9.5V & 9.2 & SB & 504 & 55 & 0. & 78 & 10 & -0 . & 0 . & 11 & 11.5 \\
\hline 5000 & 115149 & & 6.07 & .44 & F5V & 38.9 & $\mathrm{~S}$ & 221 & 0.045 & 0.018 & 11 & 65 & -0.55 & 0.33 & 24.5 & 44.3 \\
\hline 5011 & 115383 & 59 Vir & 5.22 & 0.59 & G0V s & 18.0 & VB & 331 & 1.122 & 0.087 & 415 & 5 & -0.14 & 0.07 & 849.1 & 327.3 \\
\hline 5037 & 116160 & & 5.69 & 0.06 & $\mathrm{~A} 2 \mathrm{~V}$ & 65.3 & $\mathrm{~S}$ & 307 & 0.311 & 0.036 & 189 & 7 & -0.20 & 0.10 & 225.5 & 1149.8 \\
\hline 5040 & 116235 & 64 Vir & 5.87 & 0.12 & $\mathrm{~A} 2 \mathrm{~m}$ & 63.7 & S & 293 & 0.124 & 0.024 & 55 & 2 & -0.32 & 0.18 & 82.0 & 398.1 \\
\hline 5050 & 116568 & 66 Vir & 5.75 & 0.42 & F3-4V & 30.1 & SB & 280 & 0.139 & 0.025 & 61 & 10 & -0.40 & 0.15 & 86.3 & 93.5 \\
\hline 5054 & 116656 & $\zeta \mathrm{UMa}$ & 2.27 & 0.02 & $\mathrm{~A} 1 \mathrm{VpSrSi}$ & 24.0 & SB & 545 & 0.019 & 0.008 & 8 & 54 & -0.34 & 0.36 & 12.5 & 8.6 \\
\hline 5055 & 116657 & $\zeta \mathrm{UMa}$ & 3.95 & .13 & $\mathrm{~A} 1 \mathrm{~m}$ & 24.0 & SB & 545 & 0.019 & 0.008 & 8 & 40 & -0.34 & 0.36 & 12.5 & 8.6 \\
\hline 5062 & 116842 & $80 \mathrm{UMa}$ & 4.0 & 16 & A5V & 24.9 & SB & 552 & 26 & 0. & 13 & 6 & 0.24 & 0. & 25.4 & 18.8 \\
\hline 5069 & 117025 & & 6.11 & .11 & ApSrE & 88.3 & $\mathrm{~S}$ & 209 & 0.036 & 0.015 & 10 & 27 & -0.79 & 0.23 & 14.7 & 137.2 \\
\hline 5074 & 117200 & & 6.66 & 0.37 & F0 & 67.4 & VB & 507 & 0.027 & 0.010 & 16 & 4 & -0 . & 0.30 & 21.2 & 115.3 \\
\hline 5075 & 117201 & & 7.04 & 0.39 & F0 & 65.4 & VB & 507 & 0.027 & 0.010 & 16 & 72 & -0.07 & 0.30 & 21.2 & 108.3 \\
\hline 5094 & 117661 & $73 \mathrm{Vir}$ & 6.01 & 0.18 & F0IV-V & 71.7 & VB & 334 & 0.027 & 0.012 & 8 & 36 & 0.23 & 0.44 & 26.0 & 160.2 \\
\hline 5107 & 118098 & $\zeta$ Vir & 3.37 & 0.11 & A3V & 22.4 & $\mathrm{~S}$ & 252 & 0.025 & 0.012 & 10 & 20 & -0.25 & 0.44 & 17.7 & 10.7 \\
\hline 5110 & 118216 & & 4.98 & 0.40 & F2IV & 44.5 & SB & 527 & 2.768 & 0.075 & 999 & 4 & -0.07 & 0.02 & 2197.4 & 5211.8 \\
\hline 5113 & 118261 & & 5.63 & 0.50 & $\mathrm{~F} 6 \mathrm{~V}$ & 35.5 & $\mathrm{SB}$, & 240 & 0.036 & 0.015 & 13 & 17 & -0.55 & 0.26 & 19.5 & 29.4 \\
\hline 5128 & 118646 & & 5.83 & 0.40 & F5V & 49.0 & $\mathrm{~S}$ & 275 & 0.067 & 0.019 & 17 & 29 & -0.04 & 0.26 & 54.4 & 156.1 \\
\hline 5138 & 118889 & & 5.57 & .33 & F0V & 53.5 & $\mathrm{VI}$ & 289 & 0.023 & 0.012 & 8 & 78 & -1.00 & 0.71 & 31.6 & 108.3 \\
\hline 5144 & 119055 & $1 \mathrm{Boo}$ & 5.75 & 0.01 & A1V & 92.9 & VB & 376 & 0.101 & 0.020 & 40 & 13 & 0.01 & 0.18 & 84.3 & 869.5 \\
\hline 5148 & 119124 & & 6.32 & 0.54 & F7-9V & 25.2 & VB & 541 & 0.365 & 0.029 & 372 & 5 & -0.14 & 0.07 & 276.5 & 210.5 \\
\hline 5156 & 119288 & & 6.16 & 0.42 & F3Vp & 35.8 & $\mathrm{~S}$ & 343 & 0.030 & 0.012 & 8 & 17 & -0.51 & 0.42 & 17.1 & 26.2 \\
\hline 5168 & 119756 & $1 \mathrm{Cen}$ & 4.23 & 0.38 & F3IV & 19.3 & SB & 283 & 0.615 & 0.049 & 404 & 4 & 0.02 & 0.08 & 517.9 & 230.0 \\
\hline 5185 & 120136 & $\tau$ Boo & 4.50 & 0.48 & F6IV & 15.6 & VB & 376 & 0.563 & 0.061 & 179 & 11 & -0.53 & 0.08 & 309.5 & 90.1 \\
\hline 5205 & 120602 & & 6.01 & 0.90 & K0 & 123.6 & $\mathrm{~S}$ & 311 & 0.028 & 0.012 & 11 & 15 & 0.64 & 0.36 & 33.1 & 605.4 \\
\hline 5209 & 120690 & & 6.45 & 0.69 & G5V & 19.9 & $\mathrm{~S}$ & 217 & 0.063 & 0.020 & 16 & 7 & -0.73 & 0.25 & 27.9 & 13.3 \\
\hline 5212 & 120759 & & 6.12 & 0.48 & F7V & 53.6 & VB & 295 & 0.055 & 0.017 & 11 & 21 & -0.46 & 0.33 & 32.3 & 111.0 \\
\hline 5216 & 120874 & & 6.46 & 0.09 & A3V & 82.5 & SB & 589 & & 0.013 & 49 & 9 & -0.19 & 0.16 & 54.6 & 444.9 \\
\hline 5222 & 120987 & & 5.54 & 0.44 & F4V & 50.1 & $\mathrm{SB}, \mathrm{V}$ & 310 & 0.081 & 0.019 & 36 & 16 & -0.15 & 0.22 & 60.7 & 182.2 \\
\hline 5233 & 121325 & & 6.19 & 0.53 & $\mathrm{~F} 8 \mathrm{~V}+\mathrm{G} 0$ & 33.8 & VB & 358 & 0.633 & 0.043 & 573 & 4 & 0.03 & 0.07 & 536.0 & 733.4 \\
\hline *5234 & 121336 & & 6.14 & 0.07 & A1V & 142.9 & VB & 686 & 0.047 & 0.008 & 109 & 4 & 0.27 & 0.18 & 46.2 & 1127.4 \\
\hline 5235 & 121370 & $\eta$ Boo & 2.68 & 0.58 & G0IV & 11.3 & SB & 377 & 0.144 & 0.023 & 55 & 15 & -0.65 & 0.12 & 70.0 & 10.8 \\
\hline
\end{tabular}


Table 2. continued

\begin{tabular}{|c|c|c|c|c|c|c|c|c|c|c|c|c|c|c|c|c|}
\hline $\mathrm{HR}$ & $\mathrm{HD}$ & name & V & $B-V$ & MK type & dist & binary & Exp. & $\mathrm{CR}$ & $\pm \mathrm{CR}$ & $\mathrm{Li}$ & $\Delta$ & $h r$ & $\Delta h r$ & $f_{\mathrm{x} 14}$ & $L_{\mathrm{x} 27}$ \\
\hline 5256 & 122064 & & 6.37 & 0.00 & K3V & 10.1 & $\mathrm{~S}$ & 813 & 0.016 & 0.007 & 9 & 31 & -0.75 & 0.35 & 7.2 & 0.9 \\
\hline 5257 & 122066 & $48 \mathrm{Hya}$ & 5.77 & 0.48 & F7V & 57.4 & $\mathrm{~S}$ & 229 & 0.097 & 0.022 & 37 & 23 & 0.42 & 0.22 & 102.7 & 404.6 \\
\hline 5268 & 122510 & & 6.18 & 0.48 & F8V & 38.3 & VB & 284 & 0.089 & 0.021 & 33 & 7 & -0.40 & 0.19 & 55.2 & 96.6 \\
\hline 5307 & 124115 & & 6.43 & 0.48 & F7V & 45.9 & $\mathrm{~S}$ & 406 & 0.071 & 0.015 & 38 & 19 & -0.53 & 0.16 & 38.8 & 98.0 \\
\hline *5325 & 124580 & & 6.31 & 0.60 & F9V & 21.1 & $\mathrm{~S}$ & 522 & 0.205 & 0.020 & 156 & 5 & -0.38 & 0.10 & $\begin{array}{l}129.2 \\
129.2\end{array}$ & 68.5 \\
\hline 5317 & 124425 & & 5.91 & 0.47 & $\mathrm{~F} 7 \mathrm{~V} \mathrm{w}$ & 55.7 & SB & 403 & 0.116 & 0.019 & 62 & 3 & -0.10 & 0.15 & 90.1 & 334.9 \\
\hline 5346 & 125040 & & 6.25 & 0.49 & F8V & 32.3 & VB & 428 & 0.215 & 0.026 & 148 & 8 & -0.21 & 0.10 & 154.4 & 192.4 \\
\hline 5347 & 125111 & & 6.38 & 0.36 & F2IV & 48.3 & $\mathrm{~S}$ & 541 & 0.068 & 0.013 & 43 & 11 & -0.21 & 0.17 & $\begin{array}{r}49.2 \\
4.2\end{array}$ & 137.2 \\
\hline 5350 & 125161 & $\iota$ Boo & 4.75 & 0.20 & $\mathrm{~A} 9 \mathrm{~V}$ & 29.8 & $\mathrm{SB}, \mathrm{VB}$ & 686 & 0.058 & 0.012 & 41 & 43 & -0.47 & 0.15 & 33.9 & 36.0 \\
\hline 5357 & 125283 & & 5.94 & 0.08 & $\mathrm{~A} 2 \mathrm{Vn}$ & 68.5 & $\mathrm{~S}$ & 275 & 0.052 & 0.018 & 10 & 41 & 0.42 & 0.33 & 54.5 & 305.7 \\
\hline 5365 & 125451 & 18 Boo & 5.41 & 0.38 & F5IV & 26.1 & $\mathrm{~S}$ & 234 & 0.202 & 0.033 & 70 & 10 & -0.13 & 0.16 & 153.6 & 125.1 \\
\hline 5385 & 126128 & & 6.86 & 0.43 & $\mathrm{~F} O \mathrm{~V}+\mathrm{F} 2 \mathrm{~V}$ & 65.9 & $\mathrm{SB}, \mathrm{VB}$ & 385 & 0.082 & 0.018 & 35 & 7 & -0.35 & 0.18 & 52.6 & 273.5 \\
\hline 5386 & 126129 & & 5.12 & $\begin{array}{l}-0.02 \\
-0.02\end{array}$ & A0V & 65.9 & VB & 385 & 0.082 & 0.018 & 35 & 6 & -0.35 & 0.18 & 52.6 & 273.5 \\
\hline 5387 & 126141 & & 6.22 & 0.37 & F5V & 35.7 & $\mathrm{~S}$ & 365 & 0.055 & 0.015 & 25 & 9 & -0.47 & 0.21 & 32.1 & 49.0 \\
\hline 5396 & 126354 & $\tau^{2} \mathrm{Lup}$ & 4.35 & 0.43 & $\mathrm{~F} 4 \mathrm{IV}+\mathrm{A} 7:$ & 96.3 & $\mathrm{SB}, \mathrm{VB}$ & 303 & 0.283 & 0.033 & 195 & 21 & 0.39 & 0.10 & 294.1 & 3265.6 \\
\hline 5401 & 126504 & & 5.83 & 0.31 & $\mathrm{~A} 1 \mathrm{~mA} 5 / 7-\mathrm{F} 2$ & 58.3 & $\mathrm{VB}$ & 363 & 0.022 & 0.010 & 9 & 22 & -0.56 & 0.37 & 12.0 & 48.8 \\
\hline 5404 & 126660 & $\theta$ Boo & 4.05 & 0.50 & F7V & 14.6 & $\mathrm{~S}$ & 724 & 1.996 & 0.055 & 999 & 3 & 0.02 & 0.02 & 1679.8 & 426.7 \\
\hline 5406 & 126722 & 104 Vir & 6.17 & 0.09 & A2IV & 72.3 & $\mathrm{~S}$ & 215 & 0.076 & 0.020 & 36 & 9 & -0.04 & 0.26 & 61.9 & 387.0 \\
\hline 5409 & 126868 & $\varphi$ Vir & 4.81 & 0.70 & G2IV & 41.4 & $\mathrm{SB}, \mathrm{VB}$ & 247 & 1.211 & 0.071 & 999 & 3 & 0.07 & 0.06 & 1051.3 & 2156.6 \\
\hline 5411 & 126943 & & $\begin{array}{l}7.01 \\
6.63\end{array}$ & 0.37 & F1IV & $\begin{array}{l}1.4 \\
76.5\end{array}$ & $\mathrm{~S}$ & 647 & 0.036 & 0.009 & 33 & 7 & 0.45 & 0.27 & 38.6 & 270.4 \\
\hline 5413 & 126983 & & 5.37 & 0.05 & $\mathrm{~A} 1 \mathrm{~V}+\mathrm{B}$ & 68.2 & $\mathrm{SB}, \mathrm{VB}$ & 340 & 0.165 & 0.023 & 105 & 14 & -0.15 & 0.14 & 124.0 & 690.3 \\
\hline 5426 & 127486 & & 5.87 & 0.48 & F6IV-V & 87.6 & $\mathrm{~S}$ & 311 & 0.070 & 0.018 & 20 & 31 & -0.65 & 0.17 & 34.0 & 312.0 \\
\hline 5433 & 127726 & & 6.01 & 0.22 & $\mathrm{~A} 7 \mathrm{Vn}$ & 71.3 & VB & 523 & 0.049 & 0.012 & 40 & 3 & -0.02 & 0.22 & 40.4 & 245.3 \\
\hline 5434 & 127739 & $26 \mathrm{Boo}$ & 5.92 & 0.35 & F2IV & 51.4 & $\mathrm{~S}$ & 523 & 0.024 & 0.009 & 12 & 16 & -0.19 & 0.30 & 17.7 & 56.0 \\
\hline 5436 & 127821 & 20000 & 6.09 & 0.41 & F4IV & $\begin{array}{l}31.7 \\
31.7\end{array}$ & S & 176 & 0.085 & 0.029 & 11 & 25 & 0.28 & 0.29 & 83.3 & 100.4 \\
\hline 5445 & 128093 & & 6.33 & 0.40 & F5V & 42.2 & VB & 501 & 0.089 & 0.016 & 64 & 6 & -0.13 & 0.16 & 68.2 & 145.0 \\
\hline 5447 & 128167 & $\sigma$ Boo & 4.46 & 0.36 & $\mathrm{~F} 2 \mathrm{~V}$ & 15.5 & $\mathrm{~S}$ & 457 & 0.171 & 0.022 & 112 & 4 & -0.32 & 0.11 & 112.8 & 32.3 \\
\hline 5455 & 128429 & & 6.20 & 0.46 & F5V V & 33.4 & S & 169 & 0.238 & 0.041 & 78 & $\begin{array}{l}7 \\
9\end{array}$ & -0.21 & 0.16 & $\begin{array}{l}171.1 \\
0\end{array}$ & 227.9 \\
\hline 5459 & 128620 & $\alpha^{1} \mathrm{Cen}$ & -0.01 & 0.71 & G2V & 1.3 & $\mathrm{SB}, \mathrm{VB}$ & 420 & 3.177 & 0.212 & 589 & 6 & -0.98 & 0.01 & 990.0 & 2.2 \\
\hline 5465 & 128917 & & 6.22 & 0.45 & F4V & 44.3 & $\mathrm{~S}$ & 351 & 0.040 & 0.013 & 18 & 16 & -0.23 & 0.28 & 28.2 & 66.1 \\
\hline 5468 & 129002 & $33 \mathrm{Boo}$ & 5.39 & 0.00 & $\mathrm{~A} 1 \mathrm{~V}$ & 60.4 & SB & 462 & 0.342 & 0.030 & 337 & 3 & 0.13 & 0.08 & 307.6 & 1341.9 \\
\hline 5492 & 129798 & & 6.25 & 0.41 & $\mathrm{~F} 2 \mathrm{~V}$ & 42.6 & $\mathrm{SB}, \mathrm{V}$ & 539 & 0.048 & 0.012 & 24 & 9 & -0.37 & 0.18 & 30.5 & 66.2 \\
\hline 5511 & 130109 & $109 \mathrm{Vir}$ & 3.72 & -0.01 & A0V & 39.4 & $\mathrm{~S}$ & 352 & 0.045 & 0.013 & 24 & 17 & -0.14 & 0.27 & 34.0 & 63.3 \\
\hline 5524 & 130603 & & 6.14 & 0.48 & F2-6V & 80.9 & VB & 524 & 0.033 & 0.010 & 14 & 12 & 0.11 & 0.29 & 29.1 & 227.7 \\
\hline 5530 & 130819 & $\alpha^{1} \mathrm{Lib}$ & 5.15 & 0.41 & F4IV & 23.7 & $\mathrm{~S}$ & 379 & 0.283 & 0.031 & 189 & 11 & -0.29 & 0.09 & 191.5 & 128.3 \\
\hline 5533 & 130945 & $38 \mathrm{Boo}$ & 5.74 & 0.48 & F7IV w & 46.9 & $\mathrm{~S}$ & 673 & 0.049 & 0.011 & 38 & 12 & 0.00 & 0.20 & 40.5 & 106.3 \\
\hline 5534 & 130948 & & 5.85 & 0.56 & $\mathrm{G} 0-2 \mathrm{~V}$ & 17.9 & SB & 529 & 0.407 & 0.031 & 427 & 8 & -0.34 & 0.07 & 264.6 & 101.9 \\
\hline 5544 & 131156 & $\xi \mathrm{Boo}$ & 4.55 & 0.76 & $\mathrm{G} 8 \mathrm{Ve}+\mathrm{K} 4 \mathrm{Ve}$ & 6.7 & VB & 400 & 2.440 & 0.183 & 416 & 2 & -0.31 & 0.07 & 1626.7 & 87.4 \\
\hline 5553 & 131511 & & 6.01 & 0.83 & $\mathrm{~K} 2 \mathrm{~V}$ & 11.5 & SB & 398 & 0.363 & 0.034 & 245 & 10 & -0.48 & 0.07 & 209.2 & 33.3 \\
\hline 5566 & 131923 & & 6.35 & 0.71 & G3-5V & 24.5 & $\mathrm{~S}$ & 349 & 0.017 & 0.008 & 8 & 31 & -1.00 & 0.33 & 23.0 & 16.5 \\
\hline$* 5568$ & 131977 & & 5.74 & 1.11 & $\mathrm{~K} 4 \mathrm{~V}$ & 5.9 & VB & 702 & 0.156 & 0.015 & 267 & 8 & -0.71 & 0.05 & 84.5 & 3.5 \\
\hline 5581 & 132254 & & 5.63 & 0.50 & F7V & 24.8 & $\mathrm{~S}$ & 740 & 0.027 & 0.009 & 9 & 32 & -0.10 & 0.28 & 20.7 & 15.3 \\
\hline 5596 & 133002 & & 5.6 & 0.68 & F9 & 43.3 & $\mathrm{~S}$ & 753 & 0.115 & 0.014 & 146 & 9 & -0.19 & 0.11 & 84.3 & 189.5 \\
\hline 5610 & 133408 & & 6.50 & 0.31 & FOV & 79.1 & VB & 269 & 0.047 & 0.016 & 14 & 6 & -0.43 & 0.34 & 28.6 & 213.7 \\
\hline 5612 & 133484 & & 6.65 & 0.46 & F6IV & 57.3 & VB & 785 & 0.023 & 0.007 & 15 & 14 & -0.50 & 0.24 & 12.8 & 50.3 \\
\hline 5618 & 133640 & $44 \mathrm{Boo}$ & 4.76 & 0.65 & F9-G1Vn & 12.8 & $\mathrm{SB}, \mathrm{VB}$ & 612 & 3.414 & 0.076 & 999 & 11 & -0.16 & 0.02 & 2547.5 & 496.0 \\
\hline 5630 & 134044 & & 6.35 & 0.52 & F8V & 29.5 & $\mathrm{~S}$ & 667 & 0.018 & 0.007 & 11 & 21 & 0.28 & 0.35 & 17.1 & 17.8 \\
\hline 5634 & 134083 & 45 Boo & 4.93 & 0.43 & F5 V & 19.7 & $\mathrm{~S}$ & 380 & 0.208 & 0.026 & 149 & 2 & -0.47 & 0.10 & 121.3 & 56.4 \\
\hline 5663 & 135235 & & 5.95 & 0.21 & $\mathrm{~A} 3 \mathrm{~m}$ & 51.8 & VB & 376 & 0.019 & 0.008 & 9 & 35 & -0.59 & 0.43 & 9.6 & 30.9 \\
\hline 5697 & 136347 & & 6.48 & -0.06 & A0pSi & 123.5 & VB & 378 & 0.119 & 0.019 & 72 & 7 & 0.52 & 0.15 & 132.1 & 2409.4 \\
\hline 5698 & 136351 & $\nu^{1} \mathrm{Lup}$ & 5.00 & 0.50 & F8V & 34.2 & $\mathrm{~S}$ & 392 & 0.156 & 0.022 & 110 & 4 & -0.10 & 0.14 & 121.1 & 169.2 \\
\hline 5700 & 136359 & & 5.67 & 0.48 & F7V & 41.7 & $\mathrm{~S}$ & 336 & 0.141 & 0.024 & 42 & 35 & -0.07 & 0.20 & 112.1 & 232.8 \\
\hline 5716 & 136751 & & 6.19 & 0.39 & F3-4IV s & 53.8 & $\mathrm{~S}$ & 806 & 0.021 & 0.007 & 15 & 18 & 0.06 & 0.29 & 18.6 & 64.2 \\
\hline 5719 & 136933 & $v$ Lup & 5.37 & -0.11 & $\mathrm{~A} 0 \mathrm{pSi}$ & 117.9 & $\mathrm{VH}$ & 383 & 0.105 & 0.019 & 56 & 7 & 0.16 & 0.18 & 96.3 & 1601.4 \\
\hline 5723 & 137052 & $\epsilon \mathrm{Lib}$ & 4.94 & 0.44 & F5IV & 32.4 & SB & 374 & 0.030 & 0.012 & 13 & 11 & -0.32 & 0.34 & 20.2 & 25.3 \\
\hline 5727 & 137107 & $\eta \mathrm{CrB}$ & 5.5 & 0.5 & G0V & 18.6 & $\mathrm{SB}, \mathrm{VB}$ & 376 & 0.148 & 0.024 & 76 & 2 & -0.78 & 0.09 & 61.7 & 25.6 \\
\hline 5728 & 137108 & $\eta \mathrm{CrB}$ & 6.08 & 0.00 & G3 & 18.6 & VB & 376 & 0.148 & 0.024 & 76 & 2 & -0.78 & 0.09 & 61.7 & 25.6 \\
\hline 5729 & 137333 & $\rho$ Oct & 5.57 & 0.11 & $\mathrm{~A} 2 \mathrm{~V}$ & 66.6 & $\mathrm{~s}$ & 206 & 0.268 & 0.040 & 107 & 11 & -0.14 & 0.14 & 203.1 & 1077.2 \\
\hline 5734 & 137392 & $\mu^{2}$ Boo & 6.50 & 0.59 & G1V & 37.3 & $\mathrm{~S}$ & 414 & 0.195 & 0.025 & 130 & 9 & -0.37 & 0.10 & 123.6 & 205.6 \\
\hline 5756 & 138268 & & 6.2 & 0.22 & A8 & & SB & 340 & 0.029 & 0.012 & 11 & 13 & 0.70 & 0.30 & 35.3 & \\
\hline 5758 & 138290 & & 6.57 & 0.37 & $\mathrm{~F} 4 \mathrm{~V} \mathrm{w}$ & 50.7 & $\mathrm{~S}$ & 234 & 0.106 & 0.025 & 37 & 30 & 0.13 & 0.24 & 95.1 & 292.4 \\
\hline 5765 & 138488 & & 7.00 & 0.2 & $\mathrm{~A} 3+\mathrm{F} 0 \mathrm{~V}$ & 101.6 & VB & 383 & 0.070 & 0.014 & 38 & 11 & 0.96 & 0.15 & 93.9 & 1160.5 \\
\hline 5779 & 138763 & & 6.51 & 0.58 & F7V & 25.1 & $\mathrm{~S}$ & 381 & 0.240 & 0.027 & 201 & 10 & -0.15 & 0.10 & 180.3 & 136.3 \\
\hline 5793 & 139006 & $\alpha \mathrm{CrB}$ & 2.23 & -0.02 & $\mathrm{~A} 0 \mathrm{~V}+\mathrm{G} 5 \mathrm{~V}$ & 22.9 & $\mathrm{SB}$ & 252 & 0.118 & 0.024 & 47 & 6 & -0.26 & 0.19 & 82.1 & 51.5 \\
\hline 5813 & 139389 & & 6.52 & 0.00 & F5V: & 36.0 & $\mathrm{~S}$ & 214 & 0.101 & 0.028 & 18 & 10 & -0.49 & 0.22 & 58.0 & 90.0 \\
\hline 5815 & 139460 & & 6.50 & 0.53 & F6IV-V & 22.6 & VB & 260 & 0.050 & 0.018 & 11 & 60 & -0.65 & 0.21 & 24.2 & 14.8 \\
\hline 5816 & 139461 & & 6.48 & 0.52 & F6V & 24.9 & $\mathrm{SB}, \mathrm{VB}$ & 260 & 0.050 & 0.018 & 11 & 71 & -0.65 & 0.21 & 24.2 & 17.9 \\
\hline 5818 & 139493 & & 5.7 & 0.1 & $\mathrm{~A} 2 \mathrm{~V}$ & 75.5 & VB & 1178 & 0.087 & 0.010 & 110 & 4 & -0.16 & 0.10 & 65.0 & 443.6 \\
\hline 5825 & 139664 & & 4.64 & 0.40 & F5IV-V & 17.5 & $\mathrm{~s}$ & 420 & 0.523 & 0.037 & 476 & 8 & -0.28 & 0.06 & 357.1 & 131.1 \\
\hline 5829 & 139777 & & 6.58 & 0.67 & G0IV-V+G8IV-V & 22.1 & $\mathrm{SB}, \mathrm{VB}$ & 889 & 0.785 & 0.031 & 999 & 12 & -0.07 & 0.03 & 623.0 & 362.9 \\
\hline 5830 & 139798 & & 5.75 & 0.36 & $\mathrm{~F} 2 \mathrm{~V}$ & 35.7 & $\mathrm{~S}$ & 805 & 0.017 & 0.006 & 9 & 13 & -0.28 & 0.34 & 11.7 & 17.8 \\
\hline 5845 & 140232 & $\tau^{7}$ Ser & 5.81 & 0.20 & $\mathrm{~A} 2$ & 53.2 & $\mathrm{~S}$ & 274 & 0.067 & 0.021 & 15 & 6 & -0.07 & 0.28 & 53.4 & 181.0 \\
\hline 5846 & 140285 & & 5.9 & 0.0 c & A0 & 141.4 & Vl & 375 & 0.103 & 0.020 & 50 & 20 & -0.04 & 0.17 & 83.8 & 2006.2 \\
\hline 5853 & 140538 & $\psi$ Ser & 5.88 & 0.68 & $\mathrm{G} 2.5 \mathrm{~V}$ & 14.7 & $\mathrm{SB}$ & 492 & 0.129 & 0.018 & 96 & 6 & -0.64 & 0.10 & 63.4 & 16.3 \\
\hline 5856 & 140722 & & 6.5 & 0.3 & F2IV & 68.1 & $\mathrm{VI}$ & 429 & 0.069 & 0.015 & 42 & 62 & 0.18 & 0.20 & 64.3 & 356.5 \\
\hline 5864 & 140901 & & 6.01 & 0.7 & G6 & 15 & Vl & 394 & 0.147 & 0.023 & 67 & 7 & -0.73 & 0.11 & 65.2 & 18.1 \\
\hline 5868 & 141004 & $\lambda$ Ser & 4.43 & 0.60 & G0-V & 11.8 & $\mathrm{SB}$ & 503 & 0.062 & 0.014 & 30 & 18 & -0.71 & 0.13 & 28.2 & 4.7 \\
\hline 5870 & 141187 & $v$ Ser & 5.71 & 0.09 & A3V & 77.2 & $\mathrm{~S}$ & 540 & 0.037 & 0.012 & 14 & 7 & 0.18 & 0.29 & 34.7 & 247.8 \\
\hline 5887 & 141675 & & 5.86 & 0.25 & A3m & 79.6 & $\mathrm{SB}, \mathrm{VB}$ & 1277 & 0.016 & 0.005 & 14 & 21 & -0.38 & 0.21 & 10.3 & 78.3 \\
\hline 5895 & 141851 & $36 \mathrm{Ser}$ & 5.11 & 0.12 & A3Vnp & 48.9 & $\mathrm{VB}$ & 414 & 0.028 & 0.010 & 18 & 8 & -0.38 & 0.29 & 17.8 & 50.9 \\
\hline
\end{tabular}


Table 2. continued

\begin{tabular}{|c|c|c|c|c|c|c|c|c|c|c|c|c|c|c|c|c|}
\hline HR & $\mathrm{HD}$ & name & $V$ & $B-V$ & MK type & dist & binary & Exp. & $\mathrm{CR}$ & $\pm \mathrm{CR}$ & $\mathrm{Li}$ & $\Delta$ & $h r$ & $\Delta h r$ & $f_{\mathrm{x} 14}$ & $L_{\mathrm{x} 27}$ \\
\hline 5921 & 142529 & & 6.31 & 0.38 & F1IV & 49.5 & $\mathrm{~S}$ & 359 & 0.037 & 0.013 & 16 & 15 & -0.26 & 0.29 & 25.7 & 75.3 \\
\hline 5923 & 142542 & & 6.29 & 0.43 & F6V & 50.5 & $\mathrm{~S}$ & 446 & 0.035 & 0.012 & 13 & 20 & -0.35 & 0.31 & 22.5 & 68.5 \\
\hline 5933 & 142860 & $\gamma$ Ser & 3.85 & 0.48 & F6V & 11.1 & $\mathrm{~S}$ & 560 & 0.035 & 0.012 & 10 & 9 & -0.90 & 0.13 & 12.5 & 1.9 \\
\hline 5936 & 142908 & $\lambda \mathrm{CrB}$ & 5.45 & 0.33 & FoIV & 41.5 & $\mathrm{~S}$ & 269 & 0.036 & 0.014 & 10 & 24 & -0.80 & 0.45 & 14.8 & 30.4 \\
\hline 5954 & 143333 & $49 \mathrm{Lib}$ & 5.47 & 0.52 & F8V & 32.8 & $\mathrm{SB}$ & 450 & 0.087 & 0.017 & 49 & 13 & -0.26 & 0.17 & 60.1 & 77.3 \\
\hline 5961 & 143474 & $\iota^{1}$ Nor & 4.63 & 0.24 & A7IV & 43.0 & VB & 352 & 2.045 & 0.139 & 618 & 15 & -0.08 & 0.06 & 1612.7 & 3566.3 \\
\hline 5977 & 144069 & $\xi \mathrm{Sco}$ & 5.07 & 0.00 & F5IV & & VB & 458 & 0.253 & 0.025 & 233 & 13 & -0.32 & 0.09 & 167.7 & \\
\hline 5978 & 144070 & $\xi$ Sco & 4.77 & 0.47 & F5IV & & $\mathrm{SB}, \mathrm{VB}$ & 458 & 0.253 & 0.025 & 233 & 11 & -0.32 & 0.09 & 167.7 & \\
\hline 5986 & 144284 & $\theta$ Dra & 4.01 & 0.52 & F8IV & 20.9 & $\mathrm{SB}$ & 1603 & 0.750 & 0.023 & 999 & 2 & -0.09 & 0.03 & 587.5 & 307.8 \\
\hline 5989 & 144362 & & 6.53 & 0.48 & F2IV & 78.1 & $\mathrm{SB}, \mathrm{VB}$ & 456 & 0.070 & 0.014 & 43 & 10 & 0.48 & 0.19 & 76.4 & 557.1 \\
\hline 5991 & 144415 & & 5.73 & 0.29 & F1IV & 52.0 & $\mathrm{VB}$ & 300 & 0.018 & 0.010 & 8 & 8 & -0.13 & 0.51 & 13.7 & 44.4 \\
\hline 5999 & 144668 & & 7.05 & 0.36 & A7IVe & 207.9 & VB & 417 & 0.299 & 0.030 & 158 & 70 & 0.92 & 0.04 & 394.9 & 20422.5 \\
\hline 6052 & 145976 & & 6.50 & 0.39 & F3V & 73.1 & VB & 662 & 0.032 & 0.009 & 20 & 10 & -0.59 & 0.26 & 16.4 & 105.0 \\
\hline 6063 & 146361 & $\sigma \mathrm{CrB}$ & 5.64 & 0.51 & G0VCaIIe & 21.7 & $\mathrm{SB}, \mathrm{VB}$ & 764 & 9.487 & 0.115 & 999 & 12 & 0.06 & 0.01 & 8185.4 & 4606.1 \\
\hline 6064 & 146362 & $\sigma \mathrm{CrB}$ & 6.66 & 0.00 & G1V & 21.7 & VB & 764 & 9.487 & 0.115 & 999 & 7 & 0.06 & 0.01 & 8185.4 & 4606.1 \\
\hline 6091 & 147365 & & 5.46 & 0.40 & F3IV-V & 26.8 & VB & 818 & 0.640 & 0.030 & 999 & 8 & -0.16 & 0.04 & 477.5 & 410.8 \\
\hline 6093 & 147449 & $\sigma$ Ser & 4.82 & 0.34 & FoV & 27.4 & $\mathrm{~s}$ & 439 & 0.043 & 0.013 & 14 & 7 & -0.51 & 0.30 & 24.3 & 21.8 \\
\hline 6094 & 147513 & & 5.40 & 0.62 & G5V & 12.9 & $\mathrm{~S}$ & 349 & 0.654 & 0.045 & 646 & 8 & -0.25 & 0.06 & 457.1 & 90.6 \\
\hline 6097 & 147553 & & 6.47 & 0.01 & $\mathrm{~A} 0 \mathrm{~V}+\mathrm{A} 0 \mathrm{~V}$ & 116.8 & VB & 368 & 0.074 & 0.017 & 34 & 40 & 0.01 & 0.21 & 61.8 & 1009.1 \\
\hline 6098 & 147584 & $\zeta \operatorname{Tr} \mathrm{A}$ & 4.91 & 0.55 & F9V & 12.1 & $\mathrm{SB}$ & 166 & 1.027 & 0.081 & 493 & 3 & -0.22 & 0.07 & 733.6 & 128.6 \\
\hline 6109 & 147787 & $\iota \operatorname{Tr} \mathrm{A}$ & 5.27 & 0.36 & F4IV & 40.4 & $\mathrm{SB}$, & 211 & 0.079 & 0.023 & 18 & 31 & -0.08 & 0.28 & 62.0 & 121.1 \\
\hline 6111 & 147869 & $21 \mathrm{Her}$ & 5.85 & -0.01 & A2pSr: & 104.7 & SB & 425 & 0.026 & 0.010 & 12 & 1 & 1.00 & 0.31 & 36.1 & 473.1 \\
\hline 6116 & 148048 & $\eta \mathrm{UMi}$ & 4.95 & 0.37 & F5V V & 29.8 & $\mathrm{~S}$ & 912 & 0.030 & 0.007 & 19 & 60 & -0.27 & 0.22 & 20.6 & 22.0 \\
\hline 6123 & 148283 & 25 Her & 5.54 & 0.17 & A5V & 78.6 & VB & 808 & 0.014 & 0.005 & 12 & 13 & 0.68 & 0.50 & 16.7 & 123.1 \\
\hline 6129 & 148367 & $v \mathrm{Oph}$ & 4.63 & 0.17 & $\mathrm{~A} 3 \mathrm{~m}$ & 37.5 & $\mathrm{SB}, \mathrm{VB}$ & 375 & 0.288 & 0.029 & 226 & 5 & -0.12 & 0.10 & 221.2 & 372.0 \\
\hline 6137 & 148515 & & 6.48 & 0.40 & $\mathrm{~F} 2 \mathrm{~V}$ & 46.4 & $\mathrm{SB}, \mathrm{VB}$ & 354 & 0.030 & 0.011 & 10 & 14 & -0.19 & & 216 & 55.7 \\
\hline 6162 & 149303 & & 5.65 & 0.12 & $\mathrm{~A} 4 \mathrm{Vn}$ & 69.1 & VB & 901 & 0.041 & 0.009 & 26 & 27 & -0.31 & 0.16 & 27.2 & 155.2 \\
\hline 6171 & 149661 & $12 \mathrm{Oph}$ & 5.75 & 0.82 & $\mathrm{~K} 2 \mathrm{~V}$ & 9.8 & $\mathrm{~S}$ & 603 & 0.258 & 0.032 & 148 & 4 & -0.64 & 0.09 & 126.8 & 14.5 \\
\hline 6181 & 150012 & & 6.31 & 0.41 & F5IV & 69.5 & $\mathrm{~S}$ & 473 & 0.064 & 0.014 & 31 & 17 & 0.18 & 0. & 59.6 & 344.2 \\
\hline 6186 & 150118 & 17 Dra & 6.53 & -0.06 & A1Vnn & 121.7 & $\mathrm{~S}$ & 570 & 0.072 & 0.015 & 26 & 69 & -0.95 & 0.09 & 3.6 & 418.2 \\
\hline 6212 & 150680 & $\zeta \mathrm{Her}$ & 2.81 & 0.65 & GOIV & 10.8 & $\mathrm{SB}, \mathrm{VB}$ & 494 & 0.128 & 0.019 & 77 & 19 & -0.72 & 0. & 57.4 & 8.0 \\
\hline 6216 & 150768 & & 6.58 & .10 & $\mathrm{~A} 2 \mathrm{~V}$ & 91.7 & VB & 359 & 0.040 & 0.014 & 10 & 8 & -0.58 & & 7 & 208.8 \\
\hline 6218 & 150894 & & 6.02 & 0.09 & A3IV & 231.5 & $\mathrm{VE}$ & 340 & 0.019 & 0.009 & 8 & 68 & 0.66 & 0.57 & 22.4 & 1438.3 \\
\hline 6237 & 151613 & & 4.85 & 0.38 & $\mathrm{~F} 2 \mathrm{~V}$ & 26.7 & SB & 777 & 0.235 & 0.019 & 343 & 6 & -0.02 & 0.07 & 192.8 & 164.8 \\
\hline 6240 & 151676 & & 6.10 & 0.00 & A5V & 74.2 & SB & 395 & 0.202 & 0.024 & 204 & 5 & 0.34 & & 03.9 & 1344.3 \\
\hline 6243 & 151769 & $20 \mathrm{Oph}$ & 4.65 & 0.47 & F7IV & 37.0 & $\mathrm{SB}$ & 443 & 0.163 & 0.021 & 157 & 5 & 0.00 & 0.12 & 35.3 & 221.4 \\
\hline 6254 & 152107 & $52 \mathrm{Her}$ & 4.82 & 0.09 & $\mathrm{~A} 2 \mathrm{VpS}$ & 53.7 & VB & 787 & 0.046 & 0.009 & 36 & 12 & -0.49 & 0.16 & 26.3 & 90.7 \\
\hline 6267 & 152303 & & 5.98 & 0.42 & F4V & 36.0 & VB & 1248 & 0.067 & 0.009 & 110 & 10 & 0.02 & & 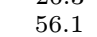 & 86.9 \\
\hline 6269 & 152311 & & 5.88 & 0.68 & G3V & 28.0 & $\mathrm{~S}$ & 344 & 0.028 & 0.011 & 9 & 19 & -0.59 & 0. & 14.4 & 13.5 \\
\hline 6279 & 152598 & $53 \mathrm{Her}$ & 5.32 & 0.29 & $\mathrm{~F} 0-2 \mathrm{~V}$ & 30.0 & $\mathrm{~S}$ & 634 & 0.065 & 0.011 & 48 & 4 & -0.08 & 0.18 & 51.0 & 55.1 \\
\hline 6302 & 153229 & & 6.59 & 0.40 & F3IV & 48.4 & $\mathrm{~S}$ & 386 & 0.029 & 0.012 & 7 & 24 & -0.20 & 0.39 & 21.2 & 59.3 \\
\hline 6310 & 153363 & $26 \mathrm{Oph}$ & 5.75 & 0.41 & F $4 \mathrm{~V}$ & 33.3 & $\mathrm{~S}$ & 274 & 0.098 & 0.022 & 44 & 6 & -0.51 & 0.18 & 54.7 & 72.5 \\
\hline 6314 & 153580 & $\epsilon^{2}$ Ara & 5.29 & 0.48 & F6V & 26.3 & VB & 243 & 0.255 & 0.036 & 100 & 6 & -0.26 & 0.14 & 176.7 & 146.6 \\
\hline 6315 & 153597 & 19 Dra & 4.8 & & & & SB & 2622 & 0.406 & & 999 & 4 & -0.42 & & & 67.2 \\
\hline 6317 & 153653 & & 6.59 & 0.23 & A7V & 73.0 & $\mathrm{SB}, \mathrm{VH}$ & 522 & 0.017 & 0.007 & 8 & 11 & -1.00 & 0.46 & 22.6 & 144.2 \\
\hline 6328 & 153897 & & 6.55 & 0.41 & F5V & 42.8 & $\mathrm{~S}$ & 632 & 0.036 & 0.009 & 27 & 19 & -0.20 & 0.24 & 26.0 & 57.2 \\
\hline 6332 & 154029 & $59 \mathrm{Her}$ & 5.25 & & & & $\mathrm{~S}$ & & 0.007 & & 7 & 24 & 0.71 & & & 86.4 \\
\hline 6349 & 154417 & & 6.01 & 0.58 & F8.5IV & 20.4 & $\mathrm{~S}$ & 496 & 0.230 & 0.023 & 193 & 2 & -0.48 & 0.08 & 132.6 & 65.9 \\
\hline 6369 & 154905 & $\mu$ Dra & 5.83 & 0 & F7V & 27.0 & VB & 1270 & 0.484 & 0.021 & 999 & 7 & -0.25 & 0.04 & 4 & 294.4 \\
\hline 6370 & 154906 & $\mu$ Dra & 5.8 & & $\mathrm{~F}$ & 27.0 & VB & 1 & 0.484 & 0.021 & 999 & 5 & -0.25 & & 4 & 294.4 \\
\hline 6375 & 155078 & & 5.56 & 0.52 & $\mathrm{~F}$ & 40.3 & $\mathrm{~S}$ & 387 & 0.075 & 0.015 & 64 & 3 & 0.46 & 0 . & 81.0 & 157.8 \\
\hline 6386 & 155379 & & 6.54 & -0.04 & A & 113.5 & $\mathrm{~S}$ & 321 & 0.035 & 0.013 & 14 & 25 & 1.00 & 0.32 & 48.0 & 740.6 \\
\hline 6400 & 155875 & & 6.53 & 0.60 & G0-1I & 38.8 & $\mathrm{~V}$ & 155 & 0.717 & 0.073 & 276 & 5 & -0.10 & 0.09 & 558.1 & 1004.8 \\
\hline 6401 & 155885 & $36 \mathrm{Oph}$ & 5.1 & & K1V & 6.0 & VI & 291 & 0.854 & 0.058 & 664 & 5 & -0.59 & 0 & .7 & 19.0 \\
\hline 6402 & 155886 & $36 \mathrm{Oph}$ & 5.0 & & $\mathrm{~K}$ & 6 & $\mathrm{SB}, \mathrm{V}$ & 291 & 0.854 & 0.058 & 664 & 6 & -0.59 & 0.05 & 442.7 & 19.0 \\
\hline 6416 & 156274 & & 5.4 & & & 0 & $\mathrm{VF}$ & 251 & 0.029 & & 8 & 43 & -0.25 & & & 1.9 \\
\hline 6426 & 156384 & & 5.9 & 1.04 & $\mathrm{~K}$ & 7.0 & $\mathrm{~V}$ & 30 & 0.206 & 0.029 & 120 & 10 & -0.60 & 0. & 105.5 & 6.1 \\
\hline 6435 & 156717 & & 6.0 & 0 & $\mathrm{~A} 2 \mathrm{Vn}$ & 114.4 & $\mathrm{VI}$ & 33 & 0.103 & 0.020 & 50 & 21 & 0.06 & 0. & 88.8 & 1390.6 \\
\hline 6436 & 156729 & $69 \mathrm{Her}$ & 4.6 & & A & & $\mathrm{S}$ & & 1 & & 28 & 14 & 0.00 & & & 92.5 \\
\hline 6469 & 157482 & & 5.51 & & & 64.4 & SE & 925 & 0.175 & 0.015 & 278 & & 0.08 & 8 & 152.8 & 757.8 \\
\hline 6489 & 157856 & & 6.44 & & $\mathrm{~F}:$ & 8 & S & 416 & 0.041 & 0.013 & 17 & 18 & 0.06 & 0. & 35.5 & 162.3 \\
\hline 6492 & 157919 & $45 \mathrm{Oph}$ & 4.2 & 0. & F5IV $\delta$ & 34.2 & $\mathrm{~S}$ & 2 & 0.037 & 0.014 & 10 & 18 & -0.61 & 0 . & 7 & 26.1 \\
\hline 6493 & 157950 & & 4.54 & & & 300 & $\mathrm{SE}$ & & 0.093 & & 62 & 7 & -0.10 & 0. & 1 & 77.9 \\
\hline 6496 & 157968 & & 6.2 & & $\mathrm{~F}^{\prime}$ & & S & 3 & 0.0 & & 48 & 21 & -0 & & 4 & 138.5 \\
\hline 6537 & 159217 & $\sigma$ Ara & 4.59 & -0.03 & $\mathrm{Ac}$ & 118.2 & $\mathrm{~S}$ & 19 & 0.041 & 19 & 10 & 10 & -0.29 & 0. & 27.6 & 461.9 \\
\hline 6538 & 159222 & & 6.5 & & $\mathrm{G}$ & 23.7 & & & 24 & 0 & 17 & 13 & -0.79 & 0 . & - & 6.8 \\
\hline 6539 & 159312 & & 6.4 & & $\mathrm{Ac}$ & 103.7 & $\mathrm{~S}$ & 297 & 0.080 & 0.019 & 37 & 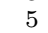 & 0.10 & 0. & 71.1 & 915.0 \\
\hline 6554 & 159541 & $\nu^{1}$ Dra & 4.88 & 0.26 & A6V & 30.3 & SB & 1674 & 0.014 & 0.004 & 15 & 64 & -0.41 & 0.23 & 8.8 & 9.7 \\
\hline 6555 & 159560 & $\nu^{2}$ Dra & 4.8 & & & & $\mathrm{SB}$ & 1674 & 0.0 & 0.004 & 15 & 2 & -0.41 & & 8.8 & 9.9 \\
\hline 6561 & 159876 & $\xi \mathrm{Se}$ & 3.5 & 0.26 & FoIV $\delta$ & 32 & $\mathrm{SB}$, & 331 & 0.8 & 0.052 & 702 & 3 & -0.01 & 0.06 & 673.0 & 841.7 \\
\hline 6569 & & $\lambda$ Ara & 4.7 & & & & $\mathrm{~S}$ & 10 & & & 32 & 10 & -0 & & & 48.7 \\
\hline 6573 & 16 & 26 Dra & 5. & & & & $\mathrm{~V}$ & 294 & 0.3 & 0.012 & 999 & 11 & -0 & 0 & 199.0 & 47.3 \\
\hline 6594 & 160910 & & 5.52 & 0.38 & F4 & 35. & VB & 609 & 0.0 & 0.013 & 90 & 0 & 0.08 & 0.16 & 76.8 & 115.9 \\
\hline 6596 & 160922 & $\omega$ Dra & 4.8 & & $\mathrm{~F}$ & 23 & $\mathrm{SB}$ & 484 & 0.465 & 0.010 & 999 & 5 & -0.28 & & 317.8 & 209.3 \\
\hline 6598 & 160933 & & 6. & & Fs & 42 & $\mathrm{~S}$ & 399 & 0.0 & 0.002 & 11 & 19 & -0.63 & 0. & 2.7 & 5.9 \\
\hline 6618 & 161693 & & 5.75 & 0.01 & $\mathrm{~A} 2 \mathrm{~V}$ & 126.6 & $\mathrm{~S}$ & 1720 & 0.0 & 0.003 & 14 & 1 & 0.54 & 0. & 10.8 & 07.7 \\
\hline 6623 & 161797 & $\mu$ Her & 3.42 & 0.75 & G5IV & 8.4 & VB & 718 & 0.197 & 0.019 & 232 & 5 & -0.86 & 0.05 & 73.9 & 6.2 \\
\hline 6636 & 162003 & $\psi^{1}$ Dra & 4.58 & 0.42 & F5IV- & 22.0 & VB & 3397 & 0.125 & 0.007 & 600 & - & -0.50 & 0.04 & 70.8 & 41.1 \\
\hline 6637 & 162004 & $\psi^{1}$ Dra & 5.79 & 0.5 & G0 & 22.3 & VB & 3397 & 0.125 & 0.007 & 600 & 23 & -0.50 & 0.04 & 70.8 & 42.2 \\
\hline 6638 & 162076 & & 5.69 & 0.94 & G5IV & 76.7 & a & 615 & 0.079 & 0.013 & 53 & 14 & 0.27 & 0.16 & 77.2 & 543.5 \\
\hline
\end{tabular}


Table 2. continued

\begin{tabular}{|c|c|c|c|c|c|c|c|c|c|c|c|c|c|c|c|c|}
\hline $\mathrm{HR}$ & $\mathrm{HD}$ & name & $V$ & $B-V$ & MK type & dist & binary & Exp. & $\mathrm{CR}$ & $\pm \mathrm{CR}$ & $\mathrm{Li}$ & $\Delta$ & $h r$ & $\Delta h r$ & $f_{\mathrm{x} 14}$ & $L_{\mathrm{x} 27}$ \\
\hline 6641 & 2132 & & 6.43 & 0.11 & $\mathrm{~A} 2 \mathrm{~V} \mathrm{~s}$ & 13 & $\mathrm{SB}, \mathrm{VB}$ & 1362 & 0.082 & 0.009 & 178 & 6 & 0.28 & 11 & 80.2 & 1661.5 \\
\hline 6656 & 162579 & 30 Dra & .02 & 0.02 & $\mathrm{~A} 2 \mathrm{~V}$ & 66.5 & VB & 1628 & 0.009 & 0.003 & 8 & 4 & -1.00 & 0.46 & 12.4 & 65.6 \\
\hline 6659 & 162596 & & 6.35 & 1.12 & KO & 136.4 & SB & 355 & 0.039 & 0.012 & 21 & 19 & 1.00 & 0.27 & 52.9 & 1178.9 \\
\hline 6670 & 162917 & & 5.77 & 0.42 & F3-5IV-V & 31.4 & SB & 339 & 0.101 & 0.020 & 49 & 2 & -0.50 & 0.17 & 57.0 & 67.1 \\
\hline 6681 & 163336 & & 5.89 & 0.05 & A1V & 76.3 & $\mathrm{VB}$ & 311 & 0.142 & 0.025 & 47 & 23 & 0.16 & 0.17 & 130.1 & 905.9 \\
\hline 6697 & 163840 & & 6.30 & 0.63 & $\mathrm{G} 2 \mathrm{~V}$ & 28.6 & $\mathrm{SB}, \mathrm{VB}$ & 651 & 0.020 & 0.007 & 14 & 30 & -1.00 & 0.33 & 27.5 & 26.8 \\
\hline 6710 & 164259 & $\zeta$ Ser & 4.62 & 0.38 & F2IV & 23.2 & SB & 343 & 0.197 & 0.025 & 133 & 2 & -0.04 & 0.13 & 159.3 & 102.5 \\
\hline 6733 & 164764 & $\tau \mathrm{Oph}$ & 5.94 & 0.00 & F5V & 52.0 & VB & 276 & 0.076 & 0.019 & 32 & 4 & -0.14 & 0.23 & 57.5 & 186.3 \\
\hline 6734 & 164765 & $\tau \mathrm{Oph}$ & 5.24 & 0.38 & $\mathrm{~F} 2 \mathrm{~V}$ & 52.0 & $\mathrm{SB}, \mathrm{VB}$ & 276 & 0.076 & 0.019 & 32 & 3 & -0.14 & 0.23 & 57.5 & 186.3 \\
\hline 6735 & 164780 & & 6.36 & 0.98 & Ko & 146.8 & $\mathrm{~S}$ & 2698 & 0.064 & 0.006 & 253 & 3 & 0.45 & 0.08 & 68.9 & 1776.9 \\
\hline 6748 & 165185 & & 5.95 & 0.62 & G5V & 17.4 & $\mathrm{~S}$ & 124 & 0.585 & 0.075 & 131 & 10 & -0.26 & 0.12 & 405.8 & 146.4 \\
\hline 6751 & 165259 & & 5.85 & 0.46 & F5V & 40.8 & VB & 139 & 0.195 & 0.041 & 47 & 16 & 0.09 & 0.21 & 171.4 & 342.3 \\
\hline 6752 & 165341 & $70 \mathrm{Oph}$ & 4.03 & 0.86 & KOV & 5.1 & $\mathrm{SB}, \mathrm{VB}$ & 347 & 1.649 & 0.127 & 410 & 7 & -0.51 & 0.06 & 924.6 & 28.6 \\
\hline 6764 & 165567 & & 6.52 & 0.46 & F7V & 50.2 & $\mathrm{~S}$ & 955 & 0.075 & 0.011 & 88 & 5 & -0.15 & 0.13 & 56.2 & 169.3 \\
\hline 6767 & 165645 & & 6.34 & 0.26 & FOV & 57.3 & $\mathrm{SB}, \mathrm{VB}$ & 1050 & 0.021 & 0.006 & 15 & 15 & -1.00 & 26 & 28.0 & 110.3 \\
\hline 6771 & 165777 & $72 \mathrm{Oph}$ & 3.73 & 0.12 & A4IV & 25.4 & $\mathrm{SB}, \mathrm{VB}$ & 401 & 0.021 & 0.010 & 7 & 27 & -0.58 & 0.30 & 11.2 & 8.6 \\
\hline 6775 & 165908 & $99 \mathrm{Her}$ & 5.04 & 0.52 & F7y & 15.7 & $\mathrm{SB}, \mathrm{VB}$ & 719 & 0.016 & 006 & 9 & 0 & -1.00 & 0.30 & 22.2 & 6.5 \\
\hline 6781 & 166045 & $100 \mathrm{Her}$ & 5.86 & 0.12 & A3V & 70.1 & VB & 633 & 0.487 & 0.029 & 754 & 7 & -0.09 & 05 & 381.2 & 2239.4 \\
\hline 6782 & 166046 & $100 \mathrm{Her}$ & 5.90 & 0.14 & A3V & 50.9 & VB & 633 & 0.487 & 0.029 & 754 & 8 & -0.09 & 0.05 & 381.2 & 1181.0 \\
\hline 6795 & 166233 & $73 \mathrm{Oph}$ & 5.73 & 0.37 & & 51.0 & v & 372 & 0.055 & & 20 & 8 & -0.39 & & 34.5 & 107.1 \\
\hline 6797 & 166285 & & 5.69 & 0.47 & F5V & 46.9 & SB & 360 & 0.047 & 0.014 & 15 & 27 & -0.28 & 5 & 32.2 & 85.0 \\
\hline 6809 & 166865 & 40 Dra & 6.04 & 0.51 & F7 & 50.9 & $\mathrm{SB}, \mathrm{VB}$ & 1564 & 0.079 & 0.008 & 158 & 4 & -0.23 & 09 & 55.9 & 173.5 \\
\hline 6810 & 166866 & 41 Dra & 5. & & $\mathrm{~F}$ & 53.1 & $\mathrm{SB}, \mathrm{VB}$ & 1564 & 0.079 & 08 & 158 & 17 & -0.23 & & & 188.6 \\
\hline 6828 & 167425 & & 6.18 & 0.58 & F & 22.9 & VB & 119 & 0.232 & 0.049 & 44 & 7 & -0.28 & 20 & 158.5 & 99.6 \\
\hline 6843 & 167833 & & 6.31 & 0.38 & $\mathrm{~A} 8 \mathrm{~V}$ & 131.9 & $\mathrm{~S}$ & 276 & 0.139 & 0.024 & 82 & 20 & 1.00 & 0.10 & 189.2 & 3939.3 \\
\hline 6844 & 167858 & & 6.63 & 0.31 & $\mathrm{~F} 2 \mathrm{~V}$ & 62.6 & $\mathrm{~S}$ & 337 & 0.029 & 11 & 10 & 11 & 0.02 & & 24.7 & 115.5 \\
\hline 6849 & 168092 & & 6.3 & 0.34 & F & 106.5 & SB & 2314 & 0.015 & 0.003 & 40 & 11 & 0.51 & & 16.5 & 224.2 \\
\hline 6850 & 168151 & 36 Dra & 5.03 & 0.38 & F5V & 23.5 & $\mathrm{~S}$ & 5748 & 0.242 & 0.007 & 999 & 4 & -0.17 & 0.02 & 179.5 & 118.6 \\
\hline 6876 & 168913 & $108 \mathrm{Her}$ & 5.63 & 0.21 & $\mathrm{~A} 5 \mathrm{~m}$ & 57.6 & SB & 687 & 0.049 & .010 & 39 & 15 & -0.17 & 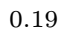 & 36.5 & 145.0 \\
\hline 6914 & 169938 & & 6.27 & 0.16 & $\mathrm{~A} 3 / 4 \mathrm{~V}$ & 82.6 & $\mathrm{~S}$ & 145 & 0.034 & 0.019 & 10 & 38 & 0.68 & t & 1.1 & 335.9 \\
\hline 6923 & 170073 & 39 Dra & 4.98 & 0.08 & $\mathrm{~A} 1 \mathrm{~V}$ & 57.8 & $\mathrm{SB}, \mathrm{VB}$ & 2349 & 0.127 & 0.009 & 400 & 83 & -0.18 & 06 & 93.4 & 373.0 \\
\hline 6927 & 170153 & $\chi$ Dra & 3.57 & 0.49 & F7V & 8.1 & $\mathrm{SB}, \mathrm{VB}$ & 2629 & 0.156 & 0.009 & 506 & 20 & -0.76 & 0.0 & 66.7 & 5.2 \\
\hline 6948 & 170773 & & 6.2 & 0. & F5 & 36.1 & $\mathrm{~S}$ & 122 & 0.138 & 0.039 & 22 & 24 & -0.25 & 7 & 96.3 & 150.0 \\
\hline 6950 & 170829 & & 6.50 & 0. & G8IV & 35.8 & SB & 553 & 0.024 & 0.008 & 14 & 21 & -0.57 & 6 & 12.6 & 19.4 \\
\hline 6956 & 170902 & & 6.37 & 0. & $\mathrm{~A} 4 \mathrm{~V}$ & 74.0 & $\mathrm{~S}$ & 305 & 0.045 & 6 & 14 & 41 & 0.02 & 0.40 & 38.0 & 248.8 \\
\hline 6981 & 171746 & & 6.2 & & G & 34.2 & $\mathrm{Vl}$ & 500 & 0.549 & 0.034 & 768 & 8 & -0.15 & 0.06 & 412.6 & 577.8 \\
\hline 6987 & 171834 & & 45 & & F3V & 31.7 & SB & 388 & 0.124 & 0.021 & 76 & 10 & -0.14 & 5 & 93.8 & 112.8 \\
\hline 6998 & 172051 & & 5. & & & 13.0 & $\mathrm{~S}$ & 283 & 0.045 & G & 15 & 10 & -0.67 & & 21.4 & 4.3 \\
\hline 7000 & 172103 & & 6 & & & 0 & $\mathrm{~S}$ & 360 & 0.030 & & 18 & 48 & 0.61 & & 34.6 & 439.4 \\
\hline 7003 & 172187 & & 6.20 & & F0V & 125.9 & $\mathrm{SB}$ & 1095 & 0.014 & 0.0 & 9 & 8 & 0.20 & 9 & 13.6 & 257.8 \\
\hline 7012 & 172555 & & 4.7 & & A5IV-V & 29.2 & $\mathrm{~S}$ & 130 & 0.097 & & 16 & 24 & -0.22 & & 69.3 & 70.8 \\
\hline 7013 & 172569 & & $6 .(1$ & & & .7 & St & 4805 & 0.010 & & 33 & 6 & -0.56 & & 5.3 & 32.6 \\
\hline 7018 & 172728 & & 5.74 & -0.0 & & 130.5 & $\mathrm{~S}$ & 2821 & 0.016 & & 45 & 12 & 0.21 & 0.18 & 14.9 & 303.6 \\
\hline 7034 & 173093 & & 6.3 & 0. & & 696 & $\mathrm{~S}$ & 344 & 0.131 & & 67 & 15 & -0.06 & & 104.9 & 608.4 \\
\hline 7038 & 173282 & & & & & & $C_{0}$ & & & & 24 & 8 & & & .4 & 236.6 \\
\hline 7047 & 173494 & & 6.31 & & $\mathrm{~F} 6$ & 44.4 & S & 458 & 0.015 & 0.007 & 8 & 26 & -1.00 & 0.60 & 20.4 & 48.2 \\
\hline 7051 & & $\epsilon^{1} \mathrm{Lyr}$ & & & & & $\mathrm{S}$ & 896 & 0.084 & 0.011 & 118 & 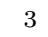 & -0.13 & & 63.9 & 189.1 \\
\hline 7052 & 173583 & $\epsilon^{1} \mathrm{Lyr}$ & 6.0 & & F1 & 49.8 & $\mathrm{n}$ & 896 & 0.084 & 0.011 & 118 & 4 & -0.13 & 0.1 & 63.9 & 189.1 \\
\hline 7056 & 173648 & $\zeta^{1} \mathrm{Lyr}$ & 4. & 0 & & 47.1 & & 631 & 0.251 & 0.022 & 286 & 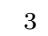 & 0.05 & 0.08 & 215.3 & 571.6 \\
\hline 7057 & 173649 & $\zeta^{2} \mathrm{Lyr}$ & 5.7 & & & 46 & & 63 & 0.251 & 0.022 & 286 & 47 & 5 & 0.08 & 215.3 & 546.1 \\
\hline 7061 & 173667 & $110 \mathrm{Her}$ & & & & $1 \mathrm{~s}$ & $\mathrm{~S}$ & & 0. & & 294 & a & -0 & & & 123.4 \\
\hline 7077 & & & & & & & & & & & 7 & 36 & & & & 649.4 \\
\hline 7123 & 175225 & & 5 & & & & S & 7 & 0.4 & 4 & 775 & 5 & -0.20 & 5 & 300.7 & 245.0 \\
\hline 712 & $1 ?$ & 50 Dra & 5. & & & & SE & 17 & & & 40 & 22 & -0 & & & 203.5 \\
\hline 712 & & & & & & & S & & & & 5 & 10 & -0 & & & 75.5 \\
\hline 7152 & 175813 & $\epsilon \mathrm{CrA}$ & 4. & & & & SB & & 0. & & 28 & 7 & -0 & & 3 & 69.9 \\
\hline 7160 & 175938 & & & & & & $\mathrm{~S}$ & 12 & & & 33 & 15 & -0 & & .1 & 243.4 \\
\hline 716 & & & & & & & & & & & 97 & 5 & & & & 11.5 \\
\hline 716 & & & 6 . & & & & S & & & & 37 & 7 & -0.36 & 0.2 & 9 & 99.7 \\
\hline 717 & & $11 \mathrm{Aql}$ & 5.2 & & & & V & & & & 81 & 5 & & & & 653.9 \\
\hline 719 & 17 & & 6. & & G & & $\mathrm{V}$ & 3000 & & & 576 & 10 & & & 9.6 & 1250.2 \\
\hline 721 & 177171 & $\rho \mathrm{Tel}$ & & & & & $\mathrm{S}$ & 127 & & (2) & 999 & 1 & 0.24 & 0.06 & 1997.8 & 6572.8 \\
\hline 721 & 17 & & & & & & $\mathrm{~S}$ & & & & 58 & 42 & & & 70.0 & 255.2 \\
\hline 721 & & 1 & & & & & 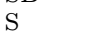 & & & & 43 & 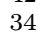 & & & & 105.3 \\
\hline 722 & & $\gamma \mathrm{C}$ & 4. & & & & SE & & & & 16 & 1 & -0 & & 5 & 7.5 \\
\hline 722 & 17 & $\gamma 0$ & 4. & & & & $\mathrm{VB}$ & 2 & & & 16 & 10 & & & 5 & 7.5 \\
\hline 723 & & & & & & & $\mathrm{~S}$ & & & & 36 & b & & & & 92.7 \\
\hline 723 & & & & & & & $\mathrm{~S}$ & & & & 7 & 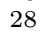 & -1 & & 4 & 11.4 \\
\hline 723 & & $\zeta \mathrm{Aql}$ & & & & & & & & & 91 & 26 & & 0 & 109.0 & 84.9 \\
\hline 724 & 178089 & & & & & & $\mathrm{~S}$ & 1418 & 0.047 & & 84 & 6 & -0 & & 36.2 & 160.0 \\
\hline 726 & & & & & & & & & & & - & 29 & & & & 16.8 \\
\hline 726 & & $17 \mathrm{Lyr}$ & & & & & & & & & 31 & 18 & & & & 40.1 \\
\hline 726 & 178476 & & 6 & 0. & & 43. & $\mathrm{~V}$ & 5 & 0.0 & & 26 & 5 & -0.91 & 0.1 & 8 & 38.6 \\
\hline 726 & & & & & & & $\mathrm{~S}$ & 4 & & & 32 & . & 5 & & 47.8 & 300.2 \\
\hline 727 & & & & & & & V & & & & 14 & 18 & -1 & 0.0 & 34.7 & 99.6 \\
\hline 7275 & 179094 & & & 1. & K1 & 70 & SE & 93 & 1.2 & 0. & 999 & 3 & 0.15 & 0.0 & 160.9 & 6849.5 \\
\hline 728 & & & & & & & S & & & & 76 & 7 & -0 & & 52.8 & 117.4 \\
\hline 72 s & & & & & & & $\mathrm{S}$ & 3 & & & 25 & 31 & -0 & 0.2 & 46.9 & 41.0 \\
\hline 731 & 180777 & 59 Dra & 5.1 & 0.3 & A9 & 27.3 & $\mathrm{~S}$ & 1584 & 0.127 & 0.0 & 351 & 6 & -0.12 & 0.07 & 97.5 & 86.9 \\
\hline 7313 & 180782 & & 6.19 & 0.02 & $\mathrm{~A} 1 \mathrm{Vn}$ & 84.5 & $\mathrm{~S}$ & 406 & 0.043 & 0.012 & 25 & 4 & -0.24 & 0.26 & 29.9 & 255.3 \\
\hline
\end{tabular}


Table 2. continued

\begin{tabular}{|c|c|c|c|c|c|c|c|c|c|c|c|c|c|c|c|c|}
\hline $\mathrm{HR}$ & HD & name & $V$ & $B-V$ & MK type & dist & binary & Exp. & $\mathrm{CR}$ & $\pm \mathrm{CR}$ & $\mathrm{Li}$ & $\Delta$ & $h r$ & $\Delta h r$ & $f_{\mathrm{x} 14}$ & $L_{\mathrm{x} 27}$ \\
\hline 7330 & 181321 & & 6.48 & 0.63 & G5V & 20.9 & $\mathrm{~S}$ & 291 & 0.627 & 0.049 & 421 & 8 & -0.08 & 0.07 & 494.1 & 257.1 \\
\hline 7345 & 181655 & & 6.31 & 0.68 & G8V & 25.2 & $\mathrm{~S}$ & 515 & 0.105 & 0.017 & 105 & 28 & -0.26 & 0.14 & 72.5 & 55.2 \\
\hline 7354 & 182101 & & 6.35 & 0.44 & F6V & 35.7 & $\mathrm{~S}$ & 498 & 0.084 & 0.014 & 75 & 2 & -0.20 & 0.16 & 61.0 & 93.2 \\
\hline 7366 & 182475 & & 6.52 & 0.33 & A9V & 100.8 & $\mathrm{~S}$ & 374 & 0.013 & 0.007 & 7 & 17 & 0.33 & 0.59 & 12.8 & 155.3 \\
\hline 7373 & 182572 & $31 \mathrm{Aql}$ & 5.16 & 0.77 & G8IVH $\delta 1$ & 15.1 & $\mathrm{~S}$ & 488 & 0.029 & 0.009 & 13 & 34 & -0.62 & 0.33 & 14.4 & 3.9 \\
\hline 7377 & 182640 & $\delta \mathrm{Aql}$ & 3.36 & 0.32 & F3IV & 15.4 & SB & 382 & 0.241 & 0.027 & 185 & 14 & -0.56 & 0.09 & 129.0 & 36.5 \\
\hline 7384 & 182761 & & 6.31 & -0.02 & A0V & 122.5 & $\mathrm{~S}$ & 381 & 0.078 & 0.017 & 31 & 24 & 0.07 & 0.20 & 67.4 & 1210.4 \\
\hline 7392 & 183007 & & 5.71 & 0.22 & $\mathrm{Am}$ & 57.4 & $\mathrm{SB}$ & 299 & 0.039 & 0.014 & 13 & 9 & -0.06 & 0.35 & 31.0 & 122.3 \\
\hline 7416 & 183806 & & 5.61 & -0.04 & $\mathrm{ApCrEuSr}$ & 133.9 & $\mathrm{~S}$ & 286 & 0.036 & 0.013 & 11 & 46 & -0.26 & 0.42 & 24.8 & 532.1 \\
\hline 7423 & 184102 & & 6.05 & 0.07 & A3V & 94.7 & $\mathrm{SB}$ & 1140 & 0.013 & 0.005 & 9 & 64 & -1.00 & 0.36 & 17.7 & 189.8 \\
\hline 7438 & 184663 & & 6.38 & 0.41 & F6IV & 42.0 & $\mathrm{~S}$ & 384 & 0.128 & 0.020 & 92 & 7 & 0.11 & 0.16 & 114.0 & 241.0 \\
\hline 7460 & 185124 & $42 \mathrm{Aql}$ & 5.46 & 0.43 & F3IV & 32.1 & $\mathrm{~S}$ & 371 & 0.093 & 0.018 & 49 & 15 & -0.17 & 0.18 & 68.8 & 84.9 \\
\hline 7462 & 185144 & $\sigma$ Dra & 4.68 & 0.79 & KOV & 5.8 & $\mathrm{~S}$ & 1448 & 0.256 & 0.014 & 694 & 1 & -0.80 & 0.03 & 104.1 & 4.1 \\
\hline 7469 & 185395 & $\theta$ Cyg & 4.48 & 0.38 & $\mathrm{~F} 4 \mathrm{~V}$ & 18.6 & VB & 853 & 0.323 & 0.021 & 562 & 7 & -0.42 & 0.05 & 196.8 & 81.4 \\
\hline 7484 & 185912 & & 5.82 & 0.44 & F6 Va & 39.8 & $\mathrm{SB}$ & 1333 & 0.123 & 0.011 & 248 & 7 & -0.30 & 0.08 & 82.7 & 156.8 \\
\hline 7496 & 186185 & & 5.49 & 0.46 & F5IV & 36.7 & $\mathrm{~S}$ & 305 & 0.100 & 0.021 & 47 & 13 & -0.44 & 0.17 & 59.6 & 96.0 \\
\hline 7528 & 186882 & $\delta$ Cyg & 2.87 & -0.03 & $\mathrm{~B} 9.5 \mathrm{IV}+\mathrm{F} 1 \mathrm{~V}$ & 52.4 & $\mathrm{SB}, \mathrm{VB}$ & 751 & 0.087 & 0.012 & 120 & 7 & 0.17 & 0.13 & 80.0 & 263.3 \\
\hline 7534 & 187013 & 17 Cyg & 4.99 & 0.47 & F7V & 20.9 & $\mathrm{VB}$ & 468 & 0.136 & 0.018 & 109 & 6 & -0.29 & 0.13 & 91.8 & 47.8 \\
\hline 7553 & 187532 & $51 \mathrm{Aql}$ & 5.39 & 0.38 & FOV & 26.4 & VB & 240 & 0.065 & 0.019 & 22 & 23 & -0.65 & 0.24 & 31.7 & 26.5 \\
\hline 7557 & 187642 & $\alpha \mathrm{Aql}$ & 0.77 & 0.22 & A7V & 5.1 & $\mathrm{~S}$ & 486 & 0.148 & 0.020 & 79 & 7 & -1.00 & 0.04 & 201.3 & 6.4 \\
\hline 7560 & 187691 & $o$ Aql & 5.11 & 0.55 & $\mathrm{~F} 8 \mathrm{~V}$ & 19.4 & VB & 401 & 0.046 & 0.013 & 16 & 21 & -0.55 & 0.24 & 25.0 & 11.3 \\
\hline 7562 & 187753 & & 6.25 & 0.10 & $\mathrm{~A} 1 \mathrm{~m}$ & 116.6 & $\mathrm{~s}$ & 428 & 0.030 & 0.010 & 13 & 14 & -0.14 & 0.37 & 22.8 & 370.2 \\
\hline 7571 & 187949 & & 6.48 & 0.14 & $\mathrm{~A} 0 \mathrm{~V}+\mathrm{F} 8 \mathrm{IV}$ & 116.6 & $\mathrm{SB}, \mathrm{VB}$ & 218 & 0.384 & 0.043 & 238 & 5 & 0.51 & 0.09 & 422.5 & 6865.9 \\
\hline 7577 & 188074 & & 6.20 & 0.36 & $\mathrm{~F} 2 \mathrm{~V}$ & 66.1 & SB & 854 & 0.074 & 0.010 & 98 & 8 & -0.14 & 0.13 & 56.4 & 294.3 \\
\hline 7578 & 188088 & & 6.18 & 1.02 & $\mathrm{~K} 2 \mathrm{~V}$ & 14.2 & $\mathrm{SB}, \mathrm{V}$ & 276 & 0.416 & 0.041 & 272 & 3 & -0.48 & 0.08 & 239.8 & 58.0 \\
\hline 7597 & 188376 & $\omega \mathrm{Sgr}$ & 4.70 & 0.75 & G5V & 23.8 & $\mathrm{SB}$ & 221 & 0.040 & 0.017 & 9 & 39 & -0.13 & 0.42 & 30.8 & 20.9 \\
\hline 7602 & 188512 & $\beta$ Aql & 3.71 & 0.86 & G8IV & 13.7 & $\mathrm{~S}$ & 343 & 0.248 & 0.029 & 202 & 12 & -0.34 & 0.10 & 161.3 & 36.3 \\
\hline 7605 & 188642 & & 6.55 & 0.39 & F4V & 52.7 & $\mathrm{~S}$ & 285 & 0.076 & 0.019 & 32 & 11 & -0.10 & 0.24 & 59.3 & 196.9 \\
\hline 7610 & 188728 & $\varphi \mathrm{Aql}$ & 5.28 & -0.01 & A1IV & 63.1 & SB & 400 & 0.039 & 0.012 & 20 & 4 & -0.32 & 0.26 & 25.6 & 122.1 \\
\hline 7631 & 189245 & & 5.66 & 0.49 & F7V & 20.9 & SB & 250 & 1.789 & 0.125 & 622 & 1 & 0.05 & 0.07 & 1534.1 & 799.9 \\
\hline 7634 & 189296 & & 6.12 & 0.00 & $\mathrm{~A} 4 \mathrm{Vn}$ & 85.7 & $\mathrm{~S}$ & 1227 & 0.010 & 0.004 & 14 & 6 & -0.12 & 0.34 & 8.0 & 70.1 \\
\hline 7665 & 190248 & $\delta \mathrm{Pav}$ & 3.56 & 0.76 & G6-8IV & 6.1 & $\mathrm{~S}$ & 124 & 0.073 & 0.028 & 10 & 60 & -0.53 & 0.41 & 40.2 & 1.8 \\
\hline 7672 & 190406 & $15 \mathrm{Sge}$ & 5.80 & 0.61 & G1V & 17.7 & $\mathrm{~S}$ & 374 & 0.056 & 0.015 & 23 & 25 & -1.00 & 0.09 & 76.2 & 28.5 \\
\hline 7674 & 190422 & & 6.26 & 0.53 & $\mathrm{~F} 8 \mathrm{~V}$ & 23.2 & $\mathrm{~S}$ & 195 & 0.282 & 0.041 & 95 & 7 & -0.34 & 0.13 & 183.9 & 118.5 \\
\hline 7683 & 190771 & & 6.19 & 0.64 & G5IV & 18.9 & VB & 710 & 0.527 & 0.028 & 970 & 3 & -0.09 & 0.05 & 412.6 & 175.8 \\
\hline 7689 & 191026 & $27 \mathrm{Cyg}$ & 5.36 & 0.85 & KOIV & 24.2 & VB & 664 & 0.336 & 0.024 & 476 & 5 & -0.27 & 0.07 & 230.9 & 161.6 \\
\hline 7692 & 191096 & & 6.21 & 0.43 & $\mathrm{~F} 4 \mathrm{~V}$ & 52.2 & $\mathrm{~S}$ & 1372 & 0.070 & 0.008 & 168 & 13 & 0.01 & 0.10 & 58.5 & 190.9 \\
\hline 7693 & 191104 & & 6.43 & 0.46 & F3V & 43.3 & $\mathrm{SB}, \mathrm{VB}$ & 297 & 0.089 & 0.020 & 32 & 12 & -0.49 & 0.18 & 50.6 & 113.3 \\
\hline 7697 & 191195 & & 5.85 & 0.39 & F5 & 36.5 & $\mathrm{~S}$ & 1175 & 0.023 & 0.006 & 21 & 11 & -0.65 & & 11.0 & 17.5 \\
\hline 7702 & 191329 & & 6.54 & 0.13 & A3V & 200.0 & $\mathrm{~S}$ & 1096 & 0.011 & 0.005 & 9 & 74 & 0.59 & 0.39 & 12.7 & 607.6 \\
\hline 7727 & 192455 & 68 Dra & 5.75 & 0.47 & F5V & 48.7 & $\mathrm{~S}$ & 1077 & 0.121 & 0.012 & 242 & 1 & -0.08 & 0.09 & 95.7 & 271.3 \\
\hline 7755 & 192983 & & 6.31 & 0.00 & $\mathrm{~A} 2 \mathrm{Vr}$ & 108.8 & VB & 1051 & 0.006 & 0.003 & 8 & 17 & -0.40 & 0.33 & 3.7 & 52.6 \\
\hline 7756 & 192985 & & 5.91 & 0.38 & F5V: & 34.5 & $\mathrm{~S}$ & 903 & 0.041 & 0.008 & 41 & 11 & -0.37 & 0.16 & 25.8 & 36.8 \\
\hline 7781 & 193592 & & 5.76 & 0.11 & $\mathrm{~A} 2 \mathrm{~V} \mathrm{~s}$ & 89.2 & $\mathrm{SB}, \mathrm{VB}$ & 1234 & 0.038 & 0.006 & 54 & 1 & -0.03 & 0.17 & 30.6 & 291.8 \\
\hline 7793 & 194012 & & 6.17 & 0.51 & & 26.1 & $\mathrm{~S}$ & 432 & 0.051 & 0.013 & 28 & 22 & -0.52 & 0.19 & 28.2 & 23.0 \\
\hline 7822 & 194943 & $\rho$ Cap & 4.78 & 0.38 & F2IV & 30.3 & $\mathrm{~S}$ & 412 & 0.098 & 0.018 & 49 & 1 & -0.56 & 0.15 & 52.1 & 57.1 \\
\hline 7826 & 195050 & 40 Cyg & 5.62 & 0.06 & A3V & 82.9 & $\mathrm{~S}$ & 621 & 0.020 & 0.008 & 9 & 32 & -0.93 & 0.25 & 6.9 & 56.9 \\
\hline 7828 & 195068 & 43 Cyg & 5.69 & 0.26 & $\mathrm{dF}$ & 37.7 & $\mathrm{~S}$ & 947 & 0.093 & 0.011 & 118 & 51 & -0.37 & 0.10 & 58.9 & 99.9 \\
\hline 7882 & 196524 & $\beta$ Del & 3.63 & 0.44 & F5IV & 29.9 & $\mathrm{SB}, \mathrm{V}$ & 520 & 0.388 & 0.041 & 214 & 5 & 0.22 & 0.10 & 367.7 & 392.2 \\
\hline 7896 & 196755 & $\kappa$ Del & 5.05 & 0.72 & G2IV & 30.1 & $\mathrm{~S}$ & 458 & 0.081 & 0.015 & 45 & 13 & -0.63 & 0.14 & 40.4 & 43.7 \\
\hline 7917 & 197120 & & 6.08 & 0.14 & $\mathrm{~A} 2 \mathrm{~V}$ & 82.0 & $\mathrm{SB}$ & 481 & 0.017 & 0.007 & 9 & 11 & 0.22 & 0.43 & 15.9 & 128.0 \\
\hline 7925 & 197373 & & 6.01 & 0.46 & F6IV & 33.2 & $\mathrm{~S}$ & 911 & 0.119 & 0.013 & 141 & 4 & -0.15 & 0.10 & 89.4 & 117.8 \\
\hline 7935 & 197649 & & 6.49 & 0.39 & F3IV & 69.3 & SB & 309 & 0.034 & 0.013 & 10 & 31 & 0.71 & 0.34 & 41.7 & 239.7 \\
\hline 7936 & 197692 & $\psi$ Cap & 4.14 & 0.43 & F $4 \mathrm{~V}$ & 14.7 & $\mathrm{~S}$ & 224 & 0.634 & 0.056 & 408 & 8 & -0.13 & 0.08 & 483.2 & 124.5 \\
\hline 7947 & 197963 & $\gamma^{1}$ Del & 5.14 & 0.49 & F7V & 31.6 & VB & 480 & 0.254 & 0.025 & 309 & 9 & 0.27 & 0.09 & 247.8 & 295.2 \\
\hline 7948 & 197964 & $\gamma^{2}$ Del & 4.27 & 1.04 & K1IV & 31.1 & VB & 480 & 0.254 & 0.025 & 309 & 2 & 0.27 & 0.09 & 247.8 & 287.0 \\
\hline 7950 & 198001 & $\epsilon \mathrm{Aqr}$ & 3.7 & 0 & A1 & 70.4 & $\mathrm{~S}$ & 432 & 0.013 & 0.007 & 7 & 26 & 1.00 & 0.79 & 17.6 & 104.0 \\
\hline 7957 & 198149 & $\eta$ Cер & 3.43 & 0.92 & K0IV & 14.3 & $\mathrm{VE}$ & 841 & 0.011 & 0.005 & 10 & 28 & -0.77 & 0.23 & 4.8 & 1.2 \\
\hline 7982 & 198571 & 4 Aqr & 5.99 & & F5V + & 58.9 & VB & 385 & 0.047 & 0.013 & 23 & 13 & -0.56 & 0.20 & 25.2 & 104.5 \\
\hline 8013 & 199260 & & 5.70 & 0.50 & F7V & 21.0 & $\mathrm{~S}$ & 375 & 0.389 & 0.034 & 352 & 5 & -0.18 & 0.08 & 285.8 & 150.8 \\
\hline 8024 & 199603 & & 6.0 & 0 & F0 & 84.5 & $\mathrm{SE}$ & 186 & 0.167 & 0.034 & 47 & 16 & 0.06 & 0.19 & 144.3 & 1234.0 \\
\hline 8028 & 199629 & $\nu \mathrm{C}$ & 3.94 & 0 . & A1 & 109.1 & $\mathrm{~S}$ & 657 & 0.048 & 0.010 & 43 & 85 & 0.61 & 0.22 & 55.4 & 788.3 \\
\hline 8034 & 199766 & $\epsilon \mathrm{Equ}$ & 5.23 & & F6IV & 60.3 & $\mathrm{SB}, \mathrm{VE}$ & 457 & 0.135 & 0.019 & 122 & 5 & 0.22 & 0.13 & 127.6 & 554.9 \\
\hline 8041 & 199960 & $11 \mathrm{Aqr}$ & 6.21 & & G & 26 & $\mathrm{~S}$ & 450 & 0.017 & 0.007 & 8 & 55 & -0.81 & 0.30 & 6.7 & 5.7 \\
\hline 8048 & 200163 & $\zeta \mathrm{Mic}$ & 5.30 & 0.41 & F3 & 35.3 & $\mathrm{~S}$ & 303 & 0.115 & 0.022 & 62 & 2 & -0.07 & 0.18 & 91.3 & 136.2 \\
\hline 8056 & 200375 & & 6.25 & & F5 & 65.9 & $\mathrm{Vl}$ & 432 & 0.075 & 0.015 & 58 & 11 & -0.16 & 0.18 & 56.1 & 291.7 \\
\hline 8060 & 200499 & $\eta$ Cap & 4.8 & & A & 48 & $\mathrm{~V}$ & 215 & 0.049 & 0.019 & 10 & 17 & -1.00 & 0.25 & 67.1 & 188.4 \\
\hline 8061 & 200525 & & 5.68 & 0.59 & F8-G0V & 18.7 & VB & 139 & 0.243 & 0.046 & 60 & 16 & -0.37 & 0.16 & 154.4 & 64.8 \\
\hline 8072 & 200740 & & 6.37 & & $\mathrm{~K}$ & 119.0 & $\mathrm{~S}$ & 810 & 0.036 & 0.008 & 50 & 5 & 0.40 & 0.21 & 38.0 & 643.7 \\
\hline 8077 & 200790 & $4 \mathrm{Eq}$ & 5.9 & & F8 & 37 & V & & 0.305 & 0.027 & 383 & 8 & -0.23 & 0.08 & 216.3 & 353.5 \\
\hline 8085 & 201091 & $61 \mathrm{Cyg}$ & 5.21 & 1.18 & K5V & 3.5 & $\mathrm{VE}$ & 543 & 0.422 & 0.030 & 452 & 10 & -0.72 & 0.04 & 189.5 & 2.8 \\
\hline 8091 & 201352 & 27 Cap & 6.25 & 0.38 & F1 & 53.9 & $\mathrm{~S}$ & 320 & 0.088 & 0.019 & 53 & 9 & 0.08 & 0.21 & 76.9 & 266.7 \\
\hline 8099 & 201636 & & 5.8 & 0. & F3. & 49.1 & $\mathrm{~S}$ & 981 & 0.027 & 0.007 & 27 & 17 & -0.29 & 0.20 & 18.3 & 52.8 \\
\hline 8100 & 201647 & & 5.83 & 0.45 & F5IV & 32.4 & $\mathrm{~S}$ & 464 & 0.119 & 0.018 & 97 & & -0.51 & 0.12 & 66.7 & 83.6 \\
\hline 8101 & 20167 & & 6. & 0. & A1 & & $\mathrm{SB}, \mathrm{VB}$ & 495 & & 0.013 & 55 & 2 & 0.07 & 0.19 & 59.0 & \\
\hline 8130 & 202444 & $\tau \mathrm{Cyg}$ & 3.7 & 0. & F2IV & 20 & $\mathrm{SB}, \mathrm{V}$ & 492 & 0.240 & 0.025 & 210 & 7 & -0.44 & 0.08 & 143.2 & 75.0 \\
\hline 8133 & 202582 & & 6.39 & 0.60 & $\mathrm{G} 2 \mathrm{IV}+\mathrm{G} 2 \mathrm{IV}$ & 41.4 & VB & 871 & 0.014 & 0.005 & 10 & 25 & -1.00 & 0.21 & 18.5 & 37.9 \\
\hline 8140 & 202730 & $\theta$ Ind & 4.39 & 0.19 & A5V & 29.8 & VB & 317 & 0.419 & 0.040 & 275 & 7 & -0.12 & 0.09 & 321.7 & 341.3 \\
\hline 8151 & 203006 & $\theta^{1} \mathrm{Mi}$ & 4.82 & 0.0 & Ap & 57.2 & $\mathrm{~S}$ & 379 & 0.106 & 0.019 & 66 & 4 & 0.30 & 0.18 & 105.1 & 411.2 \\
\hline 8162 & 203280 & $\alpha$ Cep & 2.44 & 0.22 & A7V & 15.0 & $\mathrm{~S}$ & 774 & 0.019 & 0.007 & 11 & 20 & -1.00 & 0.24 & 26.4 & 7.1 \\
\hline
\end{tabular}


Table 2. continued

\begin{tabular}{|c|c|c|c|c|c|c|c|c|c|c|c|c|c|c|c|c|}
\hline $\mathrm{HR}$ & HD & ame & V & $B-V$ & MK type & dist & binary & Exp. & CR & $\pm \mathrm{CR}$ & $\mathrm{Li}$ & $\Delta$ & $h r$ & $\Delta h r$ & $f_{\mathrm{x} 14}$ & $L_{\mathrm{x} 27}$ \\
\hline 8170 & 03454 & & 6.40 & 0.53 & $8 \mathrm{~V}$ & 26. & $\mathrm{~S}$ & 575 & 29 & .038 & 999 & 3 & -0.23 & 5 & 517.1 & 436.7 \\
\hline 8205 & 4121 & & 13 & 44 & & 47.9 & $c$ & 335 & .056 & 015 & 32 & 11 & 0.00 & 0.26 & 46.9 & 128.5 \\
\hline 8208 & 204153 & & 60 & 0.32 & FOV & 34.3 & $\mathrm{~S}$ & 702 & 0.030 & 008 & 20 & 12 & -0.62 & 0.28 & 15.3 & 21.5 \\
\hline 8210 & 204188 & & 6.07 & 0.22 & $\mathrm{~A} 8 \mathrm{~m}$ & 46.0 & $\mathrm{SB}$ & 255 & 1.052 & 0.068 & 600 & 8 & -1.00 & 0.00 & 431.8 & 631.1 \\
\hline 8220 & 204485 & & 5.80 & 0.32 & F0V & 44.6 & $\mathrm{~S}$ & 412 & 0.060 & 0.014 & 38 & 9 & -0.17 & 0.19 & 44.6 & 106.3 \\
\hline 8222 & 204577 & & 6.57 & 0.41 & F0V & 133.2 & $\mathrm{~S}$ & 376 & 0.040 & 0.013 & 14 & 24 & 0.43 & 0.30 & 42.1 & 894.0 \\
\hline 8245 & 205289 & $37 \mathrm{Ca}$ & 5.69 & 0.40 & F1V & 27.1 & $\mathrm{~S}$ & 363 & 0.192 & 0.025 & 129 & 3 & -0.42 & 0.11 & 116.9 & 103.0 \\
\hline 8263 & 205765 & & 6.25 & 0.06 & $\mathrm{~A} 2 \mathrm{~V}$ & 122.2 & VB & 375 & 0.026 & 0.011 & 9 & 46 & -0.26 & 0.38 & 18.0 & 322.3 \\
\hline 8266 & 205835 & 74 Cyg & 5.01 & 0.18 & A5V & 63.3 & $\mathrm{~S}$ & 664 & 0.014 & 0.006 & 9 & 8 & -0.54 & 0.41 & 7.6 & 36.6 \\
\hline 8276 & 206043 & & 5.85 & 0.32 & $\mathrm{~F} 2 \mathrm{~V}$ & 39.1 & G & 304 & 0.030 & 0.012 & 8 & 49 & -0.29 & 0.36 & 20.5 & 37.4 \\
\hline 8283 & 206301 & 42 Cap & 5.18 & 0.65 & $\mathrm{G} 1 \mathrm{~V}+\mathrm{G} 0 \mathrm{~V}$ & 32.5 & SB & 263 & 0.639 & 0.052 & 503 & 5 & 0.07 & 0.08 & 554.7 & 702.8 \\
\hline 8307 & 206774 & 79 Cyg & 5.65 & 0.00 & $\mathrm{~A} 0 \mathrm{~V}$ & 83.5 & $\mathrm{~S}$ & 605 & 0.439 & 0.030 & 145 & 41 & 0.66 & 0.09 & 518.1 & 4326.6 \\
\hline 8309 & 206826 & $\mu^{1}$ Cyg & 4.73 & 0.48 & F6V & 22.4 & $\mathrm{SB}, \mathrm{VB}$ & 402 & 0.155 & 0.022 & 111 & 4 & -0.17 & 0.13 & 114.6 & 68.8 \\
\hline 8310 & 206827 & $\mu^{2}$ Cyg & 6.08 & 0.36 & $\mathrm{G} 2 \mathrm{~V}$ & 22.4 & VB & 402 & 0.155 & 0.022 & 111 & 8 & -0.17 & 0.13 & 114.6 & 68.8 \\
\hline 8314 & 206860 & & 5.94 & 0.59 & G0V & 18.4 & S & 196 & 0.635 & 0.060 & 296 & 12 & -0.27 & 0.09 & 436.5 & 176.7 \\
\hline 8315 & 206901 & $\kappa \mathrm{Peg}$ & 4.13 & 0.43 & F5IV & 35.3 & $\mathrm{SB}, \mathrm{V}$ & 354 & 0.244 & 0.028 & 166 & 6 & -0.06 & 0.11 & 194.7 & 290.0 \\
\hline 8322 & 207098 & $\delta$ Cap & 2.87 & 0.29 & $\mathrm{Am}$ & 11.8 & SB & 345 & 1.655 & 125 & 479 & 5 & -0.29 & 0.07 & 1120.9 & 187.5 \\
\hline 8323 & 207129 & & 5.58 & 0.60 & G0V & 15.6 & $\mathrm{VB}$ & 334 & 0.067 & 0.017 & 25 & 10 & -0.48 & 0.21 & 38.4 & 11.2 \\
\hline 8330 & 207223 & & 6.21 & 0.34 & ערות & 50.3 & $\mathrm{~S}$ & 316 & 0.022 & 0.010 & 7 & 8 & -1.00 & & 29.5 & 89.2 \\
\hline 8363 & 208177 & & 6.20 & 0.48 & $5 \mathrm{IV}$ & 69.7 & VB & 323 & 0.054 & 0.016 & 21 & 10 & 0.20 & 0.28 & 50.5 & 293.4 \\
\hline 8369 & 208496 & $\kappa^{1}$ Ind & 6.12 & 0.46 & $3 \mathrm{~V}$ & 66.5 & SB & 375 & 0.537 & 0.039 & 480 & 13 & -0.04 & 0.07 & 434.9 & 2300.1 \\
\hline 8376 & 208703 & & 6.3 & 0.37 & F5IV & 55.6 & $\mathrm{~S}$ & 174 & 0.095 & 0.028 & 16 & 47 & -0.02 & 0.29 & 77.9 & 288.4 \\
\hline 8387 & 209100 & $\epsilon$ Ind & 4.69 & 1.06 & $\mathrm{~K} 4-5 \mathrm{~V}$ & 3.6 & $\mathrm{~S}$ & 414 & 0.260 & 0.029 & 169 & 9 & -0.85 & 0.05 & 99.1 & 1.6 \\
\hline 8400 & 209369 & $16 \mathrm{Cep}$ & 5.0 & 44 & & 37.5 & & 71 & 0.206 & & 308 & 10 & -0.04 & & & 280.5 \\
\hline 8417 & 209790 & $\xi \mathrm{Cep}$ & 4.2 & 0.34 & $\mathrm{~A} 3 / 6$ & 31.2 & $\mathrm{SB}, \mathrm{VB}$ & 644 & 0.240 & 021 & 306 & 7 & -0.42 & & 145.9 & 169.7 \\
\hline 8423 & 209942 & & 6.98 & 0.52 & F6IV- & 39.4 & $\mathrm{SB}$, & 786 & 2.607 & 0.059 & 999 & 16 & -0.04 & 0.02 & 2111.1 & 3921.2 \\
\hline 8430 & 210027 & $\iota \mathrm{Peg}$ & 3.76 & 0.44 & Y & 118 & SB & 50 & 0.260 & & 261 & 7 & -0.58 & & & 22.5 \\
\hline 8431 & 210049 & $\mu \mathrm{PsA}$ & 4.5 & 0.05 & $\mathrm{~A} 2$ & 40.0 & $\mathrm{~S}$ & 24 & 0.034 & 013 & 14 & 21 & -1.00 & & & 89.6 \\
\hline 8455 & 210460 & & 6.18 & 0.69 & G0V & 55.5 & $\mathrm{~S}$ & 402 & 0.097 & 0.017 & 71 & 10 & 0.27 & 0. & 94.4 & 348.2 \\
\hline 8457 & 210464 & & 6.09 & 0.50 & F6V & 56.7 & $\mathrm{~S}$ & 291 & .117 & & 62 & 7 & -0.05 & & & 360.7 \\
\hline 8462 & 210705 & $39 \mathrm{Aqr}$ & 6.0 & 0.38 & $\mathrm{~F} 2 \mathrm{~V}$ & 43.5 & $\mathrm{~S}$ & 268 & 0.043 & 014 & 18 & 12 & -0.34 & & & 63.3 \\
\hline 8463 & 210715 & & 5.40 & 0.15 & A5V & 54.8 & S & 511 & 0.096 & 16 & 67 & 8 & -0.37 & 0. & 61.1 & 219.6 \\
\hline 8466 & 210762 & & 5.92 & 1.51 & K0 & & $\mathrm{S}$ & 513 & 16 & & 12 & 87 & 0.83 & & & \\
\hline 8472 & 210855 & & 5.2 & 0.51 & F8V & 37.0 & $\mathrm{~S}$ & 624 & 0.111 & 014 & 156 & 8 & 0.03 & & 94 & 154.5 \\
\hline 8474 & 210884 & & 5.50 & 0.38 & $\mathrm{~F}$ & 32.7 & $\mathrm{VE}$ & 15 & 0.064 & 0.024 & 15 & 16 & -0.25 & 0. & 44 & 56.9 \\
\hline 8501 & 115 & & 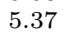 & & & 13.6 & $\mathrm{v}$ & & 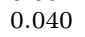 & & 12 & 11 & -1.00 & & & 12.2 \\
\hline 8507 & 211575 & & 6.3 & 0.44 & F3V & 41.5 & & $2 \varepsilon$ & 0.027 & 2 & 8 & 4 & -0.79 & & 11 & 23.3 \\
\hline 8518 & 212061 & $\gamma \mathrm{Aqr}$ & 3.84 & -0.05 & $\mathrm{~A} 0 \mathrm{~V}$ & 48.4 & SB & 24 & 0.055 & 0.017 & 22 & 15 & -0.43 & 0. & 33 & 92.9 \\
\hline 8519 & 212071 & & 6.42 & 0.00 & $\mathrm{~K} 2$ & 05.1 & $\mathrm{~S}$ & & & & 9 & 75 & 0.00 & & & 4488.8 \\
\hline 8533 & 212404 & A & 5.7 & -0.04 & & 0.0 & $\mathrm{~V}$ & & 0 & 014 & 9 & 44 & -0.17 & & 22 & 448.2 \\
\hline 8544 & 212697 & $53 \mathrm{Aqr}$ & 6.57 & 0.0 & & 20.1 & & 27 & 0.847 & 0.058 & 603 & 9 & -0.32 & 0. & 560.4 & 70.4 \\
\hline 8545 & 212698 & $53 \mathrm{Aqr}$ & 6.3 & & & & & & & 058 & 603 & 6 & -0.32 & & & 70.4 \\
\hline 8547 & 212728 & & 5.55 & 0.20 & & 43 & S & 500 & 0.012 & 0.006 & 8 & 60 & 1.00 & & 16 & 36.4 \\
\hline 8558 & 213051 & $\zeta^{1} \mathrm{Aqr}$ & 4.59 & 0.0 & $\mathrm{~F}$ & 31.7 & VB & 244 & 0.488 & 0.045 & 316 & 14 & 0.12 & 0.09 & 436.7 & 525.5 \\
\hline 8559 & 213052 & $\zeta^{2} \mathrm{Aqr}$ & 4.42 & 0.3 & $\mathrm{~F}:$ & 31.7 & $\mathrm{y}$ & 244 & 0.488 & 0.045 & 316 & 11 & 0.12 & 0.09 & 436.7 & 525.5 \\
\hline 8566 & 213235 & $37 \mathrm{P}$ & 5.4 & & 1 & 52.8 & $\mathrm{~B}, \mathrm{~V}$ & 19 & 0.0 & 13 & 7 & 74 & -0.96 & 1. & 0 & 27.2 \\
\hline 8576 & 2 & $\beta \mathrm{Ps}$ & 4.2 & & A & 45. & $\mathrm{VB}$ & 30 & 0. & & 23 & 40 & -0.52 & & 35 & 88.5 \\
\hline 8578 & 213403 & $\rho^{1} \mathrm{Cep}$ & 5.8 & & $\mathrm{~A} 2 \mathrm{~V}$ & 62.6 & $\mathrm{~S}$ & 107 & $0 . c^{-1}$ & 66 & 24 & 4 & -0.11 & 0. & 17.3 & 81.1 \\
\hline 8580 & 2134 & & 6.1 & & K0 & 145.1 & $\mathrm{~S}$ & 20 & & & 35 & 19 & 0.39 & & 8 & 097.5 \\
\hline 8581 & & & 6. & & & & $\mathrm{~V}$ & & & & 7 & 38 & o & & & 32.6 \\
\hline 8592 & 213845 & $v \mathrm{Aqr}$ & 5.2 & & & 62 & $\mathrm{~S}$ & & 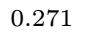 & 6 & 143 & 9 & -0.27 & & 86 & 115.5 \\
\hline 8598 & 214019 & & 6.3 & & A & 122.7 & $\mathrm{Vl}$ & 5 & 0.0 & 0.007 & 8 & 43 & -0.94 & & 5.8 & 104.9 \\
\hline 8600 & & $\sigma^{1} \mathrm{Gru}$ & 6.2 & & & & $\mathrm{C}$ & & & & 7 & 13 & -0.10 & & & 169.9 \\
\hline 8608 & 4298 & & 6 & & & 256 & & & & & 43 & 64 & -0 & & 4 & 758.3 \\
\hline 862 & 2 & & & & & & 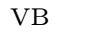 & & & & 15 & 33 & & & & 36.1 \\
\hline 8631 & & & & & & & & & & & 20 & 4 & -0.72 & & & 22.2 \\
\hline 864 & & & & & & & S & & & & 20 & 20 & & & & 982.0 \\
\hline 868 & & & & & & & V & & & & 72 & 8 & & & & 256.4 \\
\hline 8721 & & & & & & 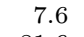 & $\mathrm{S}$ & & & & 185 & 7 & -0.47 & & & 21.4 \\
\hline 8724 & & & & & & & & & & & 26 & 3 & & & & 37.9 \\
\hline 876 & & $\pi \mathrm{Ps}$ & & & & & & & & & 109 & 5 & & & 30 & 2.1 \\
\hline 878 & 2182 & $\theta$ Gru & & & & & & & & & 23 & 6 & & & & 1.1 \\
\hline 878 & & & & & & & $\mathrm{~S}$ & & & & 59 & 6 & -0 & & & 137 \\
\hline 879 & & & & & & & $\mathrm{~S}$ & & & & 72 & 15 & & & 65 & 62.9 \\
\hline 879 & & & & & & & & & & & 13 & 5 & & & & 4.7 \\
\hline 880 & & 5 And & & & & & $\mathrm{D}$ & & & & 18 & 21 & -0 & & & 37.7 \\
\hline 881 & & & & & & & & & & & 66 & 9 & & & 168 & 241.8 \\
\hline 882 & 218804 & 6 And & & & & 28.3 & $\mathrm{~S}$ & & & & 76 & 16 & 0 & & 69.9 & 66.8 \\
\hline 883 & & & & & & a & $\mathrm{s}$ & & & & 10 & 35 & -0 . & & & 04 \\
\hline 883 & & & & & & & & & & & 11 & 8 & & & & 9.3 \\
\hline 8843 & & & & & & & $\mathrm{~S}$ & & & & 560 & 4 & & & 528 & 267.5 \\
\hline 884 & & & & & & & & & & & 18 & 6 & -0 & & & 52.3 \\
\hline 8859 & 219693 & $\varphi \mathrm{Gr}$ & 5.5 & & F5 & 34.6 & 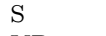 & & 0.0 & 0.025 & 12 & 4 & 0.44 & 0. & 66.7 & 95.6 \\
\hline 8865 & 01000 & $\psi^{3} \mathrm{~A}$ & 4.9 & -0.0 & & & & & & & 100 & 8 & -0 . & & 119.6 & 834.0 \\
\hline 886 & & & & & & & & & & & 13 & 19 & & & & 77 \\
\hline 886 & 2198 & $96 \mathrm{Aqr}$ & 5.5 & & & 34 & & 3 & & 0.0 & 120 & 4 & -0 & & 122.6 & 177.9 \\
\hline 887 & 2200 & & & & $\mathrm{~F} 5 \mathrm{~m} \delta \mathrm{De}$ & & VB & & & & 14 & 1 & -1 & & 00.4 & 32.5 \\
\hline & & & & & & 10 & $\mathrm{~S}$ & & & & 999 & 11 & & & 810.9 & 4175.4 \\
\hline 888 & & & 6.1 & & & 80 & $\mathrm{VE}$ & 45 & & & 13 & 32 & -0 & & 15.8 & 121.2 \\
\hline 8885 & 220117 & 12 And & 5.77 & 0.46 & F5V & 42.4 & s & 406 & 0.082 & 0.016 & 48 & 4 & -0.28 & 0.18 & 56.0 & 120.3 \\
\hline
\end{tabular}


Table 2. continued

\begin{tabular}{|c|c|c|c|c|c|c|c|c|c|c|c|c|c|c|c|c|}
\hline HR & HD & name & V & $B-V$ & MK type & dist & binary & Exp. & $\mathrm{CR}$ & $\pm \mathrm{CR}$ & $\mathrm{Li}$ & $\Delta$ & $h r$ & $\Delta h r$ & $f_{\mathrm{x} 14}$ & $L_{\mathrm{x} 27}$ \\
\hline 8888 & 220242 & & 62 & 0.37 & F5V & .1 & $\mathrm{~S}$ & 478 & 0.037 & 0.01 & 28 & 1 & -0.36 & 0.24 & 23.5 & 119.1 \\
\hline 8907 & 220729 & $o \mathrm{Gru}$ & 5.52 & 0.40 & $\mathrm{~F} 4 \mathrm{~V}$ & 2.0 & SB & 182 & 0.102 & 029 & 20 & 56 & 0.34 & .28 & 103.2 & 126.2 \\
\hline 8955 & 221970 & & 6.35 & 0.46 & F6V & 77.0 & $\mathrm{~S}$ & 326 & 0.046 & 0.015 & 17 & 19 & -0.12 & 0.29 & 35.4 & 250.8 \\
\hline 8964 & 222143 & & 6.58 & 0.66 & G5 & 23.1 & $\mathrm{~S}$ & 344 & 0.286 & 0.030 & 239 & 6 & -0.36 & 0.10 & 183.2 & 117.1 \\
\hline 8968 & 222345 & $\omega^{1} \mathrm{Aqr}$ & 5.00 & 0.24 & FOIV & 41.0 & $\mathrm{SB}$ & 240 & 0.067 & 0.019 & 22 & 10 & -0.17 & 0.27 & 49.6 & 99.9 \\
\hline 8969 & 222368 & $\iota \mathrm{Psc}$ & 4.13 & 0.51 & F7V & 13.8 & $\mathrm{~S}$ & 373 & 0.111 & 0.019 & 73 & 2 & -0.88 & 0.07 & 40.7 & 9.3 \\
\hline 8977 & 222451 & & 6.23 & 0.39 & $\mathrm{~F} 1 \mathrm{~V}$ & 44.2 & $\mathrm{~S}$ & 481 & 0.032 & 0.010 & 13 & 14 & -1.00 & 0.25 & 43.4 & 101.4 \\
\hline 9016 & 223352 & $\delta \mathrm{Scl}$ & 4.57 & 0.01 & A0V & 44.0 & $\mathrm{~S}$ & 301 & 0.198 & 0.029 & 85 & 10 & -0.01 & 0.14 & 163.8 & 379.4 \\
\hline 9020 & 223421 & & 6.33 & 0.40 & F2IV & 76.6 & $\mathrm{~S}$ & 564 & 0.048 & 0.011 & 38 & 3 & 0.55 & 0.19 & 54.1 & 380.1 \\
\hline 9028 & 223552 & & 6.44 & 0.37 & F3V & 40.5 & VB & 483 & 0.200 & 0.023 & 197 & 7 & -0.24 & 0.10 & 141.1 & 277.2 \\
\hline 9038 & 223778 & & 6.39 & 0.98 & K3V & 10.8 & SB & 747 & 0.419 & 0.025 & 795 & 8 & -0.39 & 0.05 & 261.6 & 36.4 \\
\hline 9039 & 223781 & 82 Peg & 5.30 & 0.18 & $\mathrm{~A} 4 \mathrm{Vn}$ & 57.8 & $\mathrm{~S}$ & 428 & 0.030 & 0.010 & 20 & 15 & -0.01 & 0. & 24.9 & 99.6 \\
\hline 9060 & 224361 & & 5.97 & 0.11 & A1IV & 62.9 & $\mathrm{~S}$ & 278 & 0.077 & 0.019 & 26 & 17 & 0.07 & 0.25 & 66.5 & 314.3 \\
\hline 9072 & 224617 & $\omega$ Psc & 4.01 & 0.42 & F4IV & 32.5 & $\mathrm{SB}, \mathrm{VB}$ & 431 & 0.067 & 0.015 & 34 & 17 & -0.43 & 0.21 & 40.5 & 51.1 \\
\hline 9078 & 224758 & & 6.46 & 0.50 & F7-8IV-V & 78.7 & $\mathrm{~S}$ & 277 & 0.046 & 0.015 & 19 & 14 & -0.16 & 0.30 & 34.5 & 255.9 \\
\hline 9088 & 224930 & 85 Peg & 5.75 & 0.67 & G5VbFe-2 & 12.4 & SB & 369 & 0.029 & 0.011 & 12 & 27 & -0.44 & 0.32 & 17.4 & 3.2 \\
\hline 9093 & 225003 & 32 Psc & 5.63 & 0.29 & F0V & 37.7 & $\mathrm{~S}$ & 415 & 0.077 & 0.016 & 43 & 31 & -0.47 & 0.18 & 44.8 & 76.2 \\
\hline 9095 & 225045 & & 6.25 & 0.53 & F6V & 61.9 & $\mathrm{~S}$ & 313 & 0.045 & 0.015 & 11 & 14 & 0.11 & 0.32 & 40.1 & 183.8 \\
\hline 9106 & 225233 & & 7.31 & 0.44 & $\mathrm{~F} 2 \mathrm{~V}$ & 91.6 & $\mathrm{~S}$ & 557 & 0.013 & 0.007 & 8 & 21 & -0.23 & 0.40 & 9.6 & 96.0 \\
\hline 9107 & 225239 & & 6.12 & 0.62 & G2V & 36.8 & $\mathrm{~S}$ & 262 & 0.628 & 0.052 & 489 & 1 & -0.27 & 0.07 & 432.3 & 700.2 \\
\hline
\end{tabular}

